\title{
ALBANESE KERNELS AND GRIFFITHS GROUPS
}

\author{
BRUNO KAHN
}

With an appendix by Yves André

\begin{abstract}
We describe the Griffiths group of the product of a curve $C$ and a surface $S$ as a quotient of the Albanese kernel of $S$ over the function field of $C$. When $C$ is a hyperplane section of $S$ varying in a Lefschetz pencil, we prove the nonvanishing in $\operatorname{Griff}(C \times S)$ of a modification of the graph of the embedding $C \hookrightarrow$ $S$ for infinitely many members of the pencil, provided the ground field $k$ is of characteristic 0 , the geometric genus of $S$ is $>0$, and $k$ is large or $S$ is "of motivated abelian type".
\end{abstract}

\section{Contents}

1. Introduction

Part 1. Proof of Theorem 1 11

2. Some motivic algebra 11

3. The motive of a surface (review) 12

4. Grading by coniveau 14

5. The motive of certain 3-folds $\quad 15$

6. The summands of $h_{*}(T) \quad 16$

7. $t_{3}(T)$ and the Griffiths group 19

8. The case $T=C \times S$

Part 2. Proofs of Theorems $\mathbf{2}$ and $\mathbf{3} \quad 29$

9. The $l$-adic Abel-Jacobi map 29

10. Variations of Hodge structures 32

11. Lefschetz pencils 37

12. Specialisation 43

Appendix A. A direct proof of Theorem 1

Appendix B. The Chow-Lefschetz isomorphism for $C \times S \quad 58$

Appendix C. Normality criteria, and monodromy of variations of mixed Hodge structures $\quad 59$

References 


\section{INTRODUCTION}

1.1. Cycles of higher codimension. In the late sixties, the naïve expectation that Chow groups of algebraic cycles of codimension $>1$ on smooth projective varieties should have the same structure as the Picard group was destroyed by two types of counterexamples:

Mumford: nonvanishing of the Albanese kernel $T(S)$ for most surfaces $S[71,16]$.

Griffiths: non agreement of algebraic and homological equivalence for 1-cycles on some 3-folds [42].

Mumford's theorem is the easier to prove and the more clear-cut: $T(S)$ is large when the base field $k$ is large for any surface $S$ such that $b^{2}>\rho$ (where $b^{2}$ and $\rho$ are respectively the second Betti number and the Picard number), and there is a conjectural converse (Bloch's conjecture). We recall a simple proof of Mumford's theorem in $\S 3.2$. By contrast, the nonvanishing of the Griffiths group Griff $(T)$ of a smooth projective 3 -fold $T$ has always been hard to obtain and is limited to scattered cases, with no general picture in sight, even conjectural; perhaps a reason is that a true understanding of $\operatorname{Griff}(T)$ hinges on mixed motives, rather than pure motives as in the case of $T(S)$ (compare Remark 7.11). To the best of my knowledge, $\operatorname{Griff}(T)$ is known not to vanish in the following cases, over $k=\mathbf{C}$ :

- Griffiths' original example [42]: the intersection in $\mathbf{P}^{5}$ of a hypersurface of degree $\geq 5$ with a generic hyperplane section.

- Ceresa's example [23]: the Jacobian of a general curve of genus 3. Here an explicit nonzero element of the Griffiths group is given by the Ceresa cycle.

- Schoen's examples [81]: Shimura 3-folds parametrising abelian surfaces with complex multiplication from an indefinite quaternion algebra over $\mathbf{Q}$.

- Albano's examples [2]: certain elliptic fibrations over $\mathbf{P}^{2}$.

These examples were then extended in three ways. First, Clemens [24] proved that in the example of Griffiths, $\operatorname{Griff}(T)$ is not finitely generated (even $\otimes \mathbf{Q}$ ), and Nori [75] proved the same for the example of Ceresa; see also Bardelli [11] and Voisin [95], who extended it to general Calabi-Yau 3-folds in [96] following earlier work of Paranjape [78]. Schoen and Albano directly prove infinite generation. We shall not touch this issue here, except in Remark 11.7.

Two other issues were to prove the nonvanishing of Griff $(T)$ over other fields than $\mathbf{C}$, and to give explicit rather than generic examples. Grothendieck-Katz [61] extended Griffiths' example to a (large) base 
field $k$ of arbitrary characteristic, while B. Harris $[44,45]$ used a different method to prove Ceresa's theorem, which allowed him to show that it holds (over $\mathbf{C}$ ) for the degree 4 Fermat curve $C$. Inspired by this method, Bloch [17] used arithmetic techniques to show that, over $\mathrm{Q}$, the Ceresa cycle of $C$ has infinite order in the Griffiths group of its Jacobian. For further work in this direction, see Top [91], Zelinsky [98], Tadokoro [86, 87, 88] and Otsubo [77]. Schoen proves that the Griffiths group of his examples is infinite-dimensional over $k=\overline{\mathbf{Q}}$. (I apologize for possibly missing important works in this attempt to a survey.)

In this paper, we do two things: 1) relate the Albanese kernel of a surface $S$ over the function field of a curve $C$ with $\operatorname{Griff}(C \times S)$; 2) at the prompting of Spencer Bloch, prove the nonvanishing of $\operatorname{Griff}(C \times S)$ in many cases, when $C$ is a smooth hyperplane section of $S$. For 1 ), we use the formalism of pure motives as in [82] (see Appendix 1 for a direct approach), while 2) uses known results on the variations of various tannakian groups in families.

1.2. Main results. Let $C$ (resp. $S$ ) be a smooth, projective, geometrically connected curve (resp. surface) over a field $k$. For any extension $E / k$, write $T\left(S_{E}\right) \subset C H_{0}\left(S_{E}\right)$ for the Albanese kernel of $S$ over $E$. We work in the category $\mathbf{A} \mathbf{b} \otimes \mathbf{Q}$ of abelian groups up to isogeny, i.e. the localisation of the category $\mathbf{A b}$ of abelian groups by the Serre subcategory of abelian groups of finite exponent; see [53, Rem. 4.13], [51, §2] and [10, App. B].

Theorem 1. There is a natural surjection in $\mathbf{A b} \otimes \mathbf{Q}$

$$
T\left(S_{k(C)}\right) / T(S) \longrightarrow \operatorname{Griff}(C \times S)
$$

where, for any 3-fold $X, \operatorname{Griff}(X)$ denotes the group of numerically trivial cycles of codimension 2 on $X$, modulo algebraic equivalence.

(See Remark 8.4 b) for a conjectural description of the kernel of (1.1) as the rational points of a certain abelian variety.)

Note that this numerical Griffiths group coincides in $\mathbf{A b} \otimes \mathbf{Q}$ with the usual one for 3-folds in characteristic 0, thanks to Lieberman's results [67, Cor. 1]; in positive characteristic and for $l$-adic cohomology, Lieberman's arguments go through when the Tate conjecture is known.

We give two proofs of Theorem 1 . The first one is motivic and based on an extension of the computations of [56] to 3-folds. Namely, it is a consequence of the formulas in $\mathbf{A b} \otimes \mathbf{Q}$ :

$$
\begin{aligned}
T\left(S_{k(C)}\right) / T(S) & \simeq \mathcal{M}\left(h_{1}(C), t_{2}(S)\right) \\
\operatorname{Griff}(C \times S) & \simeq \mathcal{M}_{\mathrm{alg}}\left(h_{1}(C), t_{2}(S)\right)
\end{aligned}
$$


where $\mathcal{M}$ (resp. $\mathcal{M}_{\text {alg }}$ ) denotes the category of Chow motives (resp. of motives modulo algebraic equivalence), and $t_{2}(S)$ is the transcendental part of the motive of $S$ which was studied in [56].

The second proof is essentially by hand and does not assume $\operatorname{dim} S=$ 2, see Appendix A. This proof gives more careful control on the integers which are implicit in Theorem 1. As a byproduct of this second proof, we get:

Corollary 1. Suppose that $k$ is a finite field and that $S_{\bar{k}}$ is of abelian type: its Chow motive is a direct summand of the motive of an abelian variety. Then the group $T\left(S_{k(C)}\right)$ is finite.

For a surface, to be of abelian type is a birational invariant. Examples include abelian surfaces, products of two curves and Fermat surfaces (Katsura-Shioda).

For a general $k$, we also have:

Corollary 2. Let $\Omega$ be a universal domain over $k$. If $T\left(S_{\Omega}\right)=0$, then the abelian group $\operatorname{Griff}(C \times S)$ has finite exponent for any $C$. (If $k$ is a universal domain over its prime subfield, the condition $T(S)=0$ is sufficient.)

In Corollary A.11, we show that one can choose the finite exponent independent of $k$, and dependent only on the index of $C$ (i.e., the gcd of the degrees of its closed points).

If $k$ is algebraically closed, Corollary 2 is a special case of $[18$, Th. 1 (ii)]. Thus, Corollary 2 is not really new. The converse question: when is $\operatorname{Griff}(C \times S)$ non torsion? is more interesting. We tackle it in the following special case:

Suppose $C$ is a smooth hyperplane section of $S$, and let $\psi: C \hookrightarrow S$ be the corresponding closed immersion. Modifying the graph of $\psi$ appropriately, we get a class

$$
\psi_{\#} \in T\left(S_{k(C)}\right) / T(S) \otimes \mathbf{Q}
$$

hence also a class in $\operatorname{Griff}(C \times S) \otimes \mathbf{Q}$, in view of (1.1).

Theorem 2. Suppose char $k=0$ and $p_{g}>0$. Let $f: \tilde{S} \rightarrow \mathbf{P}^{1}$ be a Lefschetz pencil of hyperplane sections of $S$, and let $U \subset \mathbf{P}^{1}$ be the open subset over which $f$ is smooth. For $u \in U$, write $\psi_{u}: C_{u}=f^{-1}(u) \hookrightarrow S$ for the closed immersion of the corresponding fibre, and write $\operatorname{Cnd}(u)$ for the condition

$$
\left(\psi_{u}\right)_{\#} \neq 0 \text { in } \operatorname{Griff}\left(C_{u} \times_{k(u)} S_{k(u)}\right) \otimes \mathbf{Q} .
$$

Then: 
(i) (Corollary 11.6) $\operatorname{Cnd}(\eta)$ holds, where $\eta$ is the generic point of $U$.

(ii) (Theorem 12.5) If $k=\mathbf{C}$, the set of $u \in U(k)$ such that $\operatorname{Cnd}(u)$ holds is uncountable.

For each $u$ for which we can prove that $\operatorname{Cnd}(u)$ holds, a by-product of the proof yields that the motivic intermediate Jacobian of $C \times S$ defined in Lemma 5.1 has no exceptional summand (see Definition 8.7 and Theorem $11.3 \mathrm{~d})$ ), and that the $l$-adic representation $H_{c, l}^{2}\left(\left(S_{k(u)}-C_{u}\right) \overline{k(u)}\right)$ is "genuinely mixed", see Proposition 9.4: this may be of independent interest. It is likely that a similar argument yields that the Hodge structure $H_{c, B}^{2}\left(\left(S_{k(u)}-C_{u}\right)_{\mathbf{C}}\right)$ is also "genuinely mixed" when $k$ is a subfield of $\mathbf{C}$ : we leave to the reader the pleasure to fill in details.

If one believes in Bloch's conjecture, Corollary 2 implies that the hypothesis $p_{g}>0$ is necessary in Theorem 2. Under a stronger hypothesis on $S$, we get a stronger result (see Theorem 12.21 for a sharper statement):

Theorem 3. Suppose that $S_{\bar{k}}$ is of motivated abelian type (Definition 12.7). If $k$ is finitely generated over $\mathbf{Q}$ (resp. in general), the set of $u \in U(k)$ such that $\operatorname{Cnd}(u)$ fails is finite (resp. countable).

Examples include: (a) the examples after Corollary 1, (b) K3 surfaces, (c) surfaces of general type verifying $p_{g}=K^{2}=1$. See Examples 12.8 for more details.

Theorems 2 and 3 prompt:

Question. Are there examples where $\operatorname{Cnd}(u)$ fails?

In [51, p. 1049], it was imprudently asserted without proof that $\psi_{\#}=$ 0 in $\mathcal{M}\left(h_{1}(C), t_{2}(S)\right)$; I thank Rémi Lodh for drawing my attention to this issue. It follows from Theorems 2 and 3 that the values of $A$ and $B$ in Row $i=2$ of the bottom table of [51, p. 1047] are not justified; the other results of [51] are not affected. It would be very interesting to understand the correct values of $A$ and $B$ : by Poincaré duality, this is related to Suslin homology of the open complement. I hope to come back to this question later.

1.3. Comments on the proofs. In Theorem 2, if we were only interested in the nonvanishing of $\psi_{\#}$ in $T\left(S_{k(C)}\right)$, we could content ourselves to quote the paper of Green, Griffiths and Paranjape [41, Th. 2]. The added difficulty for working in $\operatorname{Griff}(C \times S)$ is that the Abel-Jacobi map used in [41] does not factor through algebraic equivalence in general. This happens, however, when the first step of the coniveau filtration vanishes on the relevant cohomology group (Proposition 9.1), and this 
is the extra condition that we have to achieve: it is done in Section 10, which uses André's results on the variation of the Mumford-Tate group in families [4]; the main point is Corollary 10.8.

Similarly, Green-Griffiths-Paranjape obtain Theorem 3 when replacing $\operatorname{Griff}(C \times S)$ with $T\left(S_{k(C)}\right)$ and "finite" with "thin" in the sense of Serre [83, §9], while they don't need the abelian type hypothesis. For this, they use a specialisation argument due to Serre and Terasoma, which relies on Hilbert's irreducibility theorem. It turns out that this argument is not sufficient to handle the coniveau issue. For this, a decisive input is André's theory of motivated cycles [6], notably his refinements of Deligne's theorems from [31] and his result on the variation of the motivic Galois group in families [6, Th. 5.2]. Recent results of Cadoret-Tamagawa on the variation of $l$-adic representations in families $[21,22]$ then allow us to refine "thin" to "finite".

Suppose $k$ finitely generated over Q. It follows from André's Th. 5.23 ) in [6] that the rank of the (geometric) Néron-Severi group of a smooth projective family over $k$ is equal to the generic rank outside a thin subset of the base. (This result goes back to Terasoma in a special case [89].) Using her results with Tamagawa, Cadoret refined this, as above, by replacing "thin" with "finite' [19, Cor. 5.4]. One can consider part of the present work as a higher analogue of these results, relying on cohomological degree 3 rather than 2 .

Finally, while the arguments of Green-Griffiths-Paranjape also work in characteristic $p>0$ as long as $k$ is not algebraic over $\mathbf{F}_{p}{ }^{1}$, the proofs of Theorems 2 and 3 are firmly rooted in characteristic 0 , because Hodge theory is used in an essential way in 3 different places: Theorem 10.1 (the theorem of the fixed part of Steenbrink-Zucker), Lemma 10.6 (Hodge coniveau 0 is stable under tensor product) and Lemma 11.2 (the Lefschetz $(1,1)$ theorem). Characteristic 0 is also used in the results of Cadoret-Tamagawa (see however recent work of Emiliano Ambrosi [3]). So the issue of extending the present results to positive characteristic is interesting and open; among the uses of Hodge theory just mentioned, the least obvious to transpose to l-adic cohomology is Lemma 10.6, which is false in positive characteristic because of the supersingular elliptic curves.

In comparison, few of the examples mentioned in $\S 1.1$ work in positive characteristic. For Griffiths' original example, one needs primitive cohomology of the given complete intersection not to be fully of coniveau $>0$. In characteristic 0 , this follows easily from Hodge theory

\footnotetext{
${ }^{1} \mathrm{Up}$ to checking the correct version of Hilbert's irreducibility theorem in positive characteristic, see [38, Ch. 12 and 13].
} 
as in [26, Cor. 2.8]; but in order to obtain it in characteristic $>0$ for [61], Katz had to prove a weaker, generic result in [62, Th. 4.1], whose proof uses zeta functions of varieties over finite fields. Bloch's proof for [17], in turn, uses specialisations to finite fields, hence transposes naturally from number fields to global fields of positive characteristic as in Zelinsky [98]. It is worth asking whether either of these methods is relevant here.

1.4. Contents of this paper. The first 8 sections are computations in the categories of pure motives over $k$ [82]. In $\S 2$, we recall basic computations of Hom groups in these categories. $\S 3$ recalls some results from [56], including a simple proof of the theorem of Mumford and Bloch that $T(S)$ is "huge" for a surface $S$ with $b^{2}>\rho$ over a large algebraically closed field.

$\S \S 4$ and 5 establish a refined Chow-Künneth decomposition, first introduced in [56, Th. 7.7.3], for 3-folds $T$ admitting a Chow-Künneth decomposition; while its construction for surfaces in [56] was geometric, the present one is purely category-theoretic (of course, this method also works for surfaces). (See also related papers of Vial [92, 93].) In particular, we have a decomposition

$$
h_{3}(T) \simeq t_{3}(T) \oplus h_{1}\left(J^{2}\right)(1)
$$

where $J^{2}=J^{2}(T)$ is a certain abelian variety and $t_{3}(T)$ is "the" transcendental part of $h_{3}(T)$. By construction, this decomposition is unique up to unique isomorphism modulo numerical equivalence; in particular, $J^{2}$ is well-defined up to isogeny. Let us stress however that, contrary to the case of surfaces, it is by no means clear that (5.4) is unique modulo rational equivalence, even up to isomorphism (this would follow from the conjectures of Bloch-Beilinson and Murre [47], see also Lemma 7.5).

In $\S 6$, we study $J^{2}$. We show that it satisfies two dual universal properties (Lemma 6.3) and is isogenous to Murre's algebraic representative of [72] (Proposition 6.4) when $k$ is perfect; see also Corollary 12.22.

In $\S 7$, under the assumption that $T$ verifies a "Chow-Lefschetz isomorphism" in degree 2, we establish the basic formula

$$
\operatorname{Griff}(T) \simeq \mathcal{M}_{\mathrm{alg}}\left(\mathbb{L}, t_{3}(T)\right)
$$

for any decomposition (5.4): this completes the proof of [57, Th. 4.3.3] in a slightly broader setting. By Theorem B.1, the Chow-Lefschetz hypothesis holds in particular for the product of a curve and a surface. We further conjecture in Conjecture 7.8 that $\mathcal{M}\left(\mathbb{L}, t_{3}(T)\right) \stackrel{\sim}{\rightarrow}$ $\mathcal{M}_{\text {alg }}\left(\mathbb{L}, t_{3}(T)\right)$, and prove in Proposition 7.9 that this is a consequence of the Bloch-Beilinson-Murre conjectures (see Remark 7.11 for further 
comments on Conjecture 7.8). We deduce Formula (8.1) (see §1.2) from (7.4) in $\S 8$ (the special case $T=C \times S$ ).

Sections 9 to 12 are devoted to the proofs of Theorems 2 and 3. In Proposition 9.1, we show that the (l-adic) Abel-Jacobi map factors through algebraic equivalence if one factors out the coniveau $\geq 1$ part $N^{1}$ of cohomology (Proposition 9.1). $§ 10.1$ contains some results on variations of (pure) Hodge structures; the most important, Corollary 10.8, says that the generic vanishing of $N^{1}$ specialises outside a countable subset under suitable hypotheses. Corollary 11.6 is the generic part of Theorem 2; we also find an intriguing surjection (11.5) in $\S 11$. Section 12 is devoted to specialising this result: Theorem 2 (ii) is proven in $\S 12.1$ (Theorem 12.5), $\S 12.2$ reviews André's motives and introduces the condition "of motivated abelian type" (Definition 12.7), §12.3 proves a technical result on the coniveau filtration (Theorem 12.12), §12.4 studies $l$-adic representations and draws some consequences of results of Cadoret-Tamagawa (Theorem 12.14), §12.5 proves two technical lemmas, and finally Theorem 3 is proven in $\$ 12.6$ under a more precise form (Theorem 12.21).

There are 3 appendices. The first one gives a cycle-theoretic proof of Theorem 1, relying on the study of the Albanese map on 0-cycles initiated by Ramachandran [79] and developed in [57]. We prove Corollary 1 there. The second one shows that a 3 -fold of the form $C \times S$ verifies the "Chow-Lefschetz isomorphism in degree 2" alluded to above. The last one, by Yves André, gives a proof of Theorem 10.1, using (for the first time) the concept of observability in the tannakian setting.

1.5. Acknowledgements. I thank Claire Voisin for mentioning the article [41] of Green-Griffiths-Paranjape in the early stages of this work. Inspiration from [41] will be obvious in $§ 11$; I also drew inspiration from Katz's exposé on Griffiths' theorem in SGA 7 [61]. I thank Spencer Bloch for ignoring Corollary 2, but asking the right question which led to Theorems 2 and 3. Thanks also to Wayne Raskind for asking a question which led to Corollary 1. I thank Yves André and Anna Cadoret as well for their generous help, including numerous exchanges without which completing the proofs of these theorems would have been hazardous (in a French or an English sense). I am especially grateful to Yves André for pointing out a gap in a (perhaps too optimistic) version of Theorem 10.1, and for accepting to add an appendix to fill this gap. Finally, I thank the referee for several helpful comments, especially for pointing out a reference I had missed in [48]. 
1.6. Notation. We fix the base field $k$ and denote by $\operatorname{Sm}^{\text {proj }}(k)=$ $\mathbf{S m}^{\text {proj }}$ the category of smooth projective $k$-varieties, with morphisms the morphisms of $k$-schemes. Unless otherwise specified, variety means smooth projective variety; similarly for curve, surface, 3-fold. . .

For any adequate equivalence relation $\sim$ on algebraic cycles [80], we write $\mathcal{M}_{\sim}(k)=\mathcal{M}_{\sim}$ for the category of effective pure motives modulo $\sim$ with $\mathrm{Q}$ coefficients: this is the largest full subcategory of the category of [82], denoted here by $\mathcal{M}_{\sim}\left[\mathbb{L}^{-1}\right]$, which contains all motives of smooth projective varieties and is stable under direct sums and direct summands. We will work only occasionally in $\mathcal{M}_{\sim}\left[\mathbb{L}^{-1}\right]$ to apply Poincaré duality.

We simply write $\mathcal{M}$ if $\sim$ is rational equivalence, and write $\mathcal{M}_{H}$ if $\sim$ is homological equivalence relative to a Weil cohomology $H$. If $X$ is a smooth projective variety, we write $h(X)$ for its motive in $\mathcal{M}_{\sim}$, and only write $h_{\sim}(X)$ if there is a risk of confusion. We use the covariant convention, as in [56] and unlike [82]: the functor $h: \mathbf{S m}^{\text {proj }} \rightarrow \mathcal{M}_{\sim}$ is covariant. If $\mathbb{L}$ is the Lefschetz motive and $M \in \mathcal{M}_{\sim}$, we abbreviate $M \otimes \mathbb{L}^{\otimes n}$ to $M(n)($ not $M(-n)$ as in [82]).

As in $[51, \S 2]$, we use refined Hom groups with values in the category $\mathbf{A b} \otimes \mathbf{Q}$ of abelian groups up to isogeny; by abuse, we keep the notation $\mathcal{M}_{\sim}(-,-)$ for these refined Hom groups, and similarly for $\mathbf{D} \mathbf{M}_{\mathrm{gm}}^{\mathrm{eff}}(-,-)$ in Subsection 7.4.

For $X \in \mathbf{S m}^{\text {proj }}$ and $i \geq 0$, we write $A_{\sim}^{i}(X)$ (resp. $\left.A_{i}^{\sim}(X)\right)$ for the group of cycles of codimension (resp. dimension) $i$ on $X$, modulo $\sim$, viewed as an object of $\mathbf{A b} \otimes \mathbf{Q}$ (see above). We shall use without further mention the formulas (opposite to $[82,2.1]$ ):

$$
A_{\sim}^{i}(X)=\mathcal{M}_{\sim}\left(h(X), \mathbb{L}^{i}\right), \quad A_{i}^{\sim}(X)=\mathcal{M}_{\sim}\left(\mathbb{L}^{i}, h(X)\right) .
$$

In particular, these groups are 0 for $i \notin[0, \operatorname{dim} X]$. As in $[82$, loc. cit.], we use these formulas to extend the functors $A_{\sim}^{i}, A_{i}^{\sim}$ to all $M \in$ $\mathcal{M}_{\sim}$.

A Weil cohomology is classical if it belongs to the following list: $l$-adic cohomology in any characteristic $\neq l$, Betti or de Rham cohomology in characteristic 0 and crystalline cohomology in characteristic $>0$ [8, Def. 4]. All these cohomology theories verify

$$
\operatorname{dim} H^{1}(X)=2 \operatorname{dim} P_{X}
$$

where $P_{X}$ is the Picard variety of $X \in \mathbf{S m}^{\text {proj }}$ (cf. [65, Cor. 2A10]). More generally, the Betti numbers of $X$ relative to $H$ do not depend on the choice of $H$ : this follows from the comparison theorems in characteristic 0 and from $[29,63]$ in characteristic $>0$. 
We shall use repeatedly the Chow-Künneth decomposition of Murre [47, Conj. 5.1 (A)] and the refined Chow-Künneth decomposition of [56, (7.12)] (abbreviated respectively CK and refined CK decomposition). When they exist, the first one lifts the Künneth decomposition of the motive of a smooth projective variety from homological to rational equivalence, and the second one does the same with respect to a finer decomposition incorporating coniveau.

We use the notation $\approx$ to denote isogenies between abelian varieties. 


\section{Part 1. Proof of Theorem 1}

\section{Some motivic Algebra}

Let $X \in \mathbf{S m}^{\text {proj }}$. By Murre [73, 82], we have a partial Chow-Künneth decomposition

$$
h(X)=h_{0}(X) \oplus h_{1}(X) \oplus h_{>1}(X)
$$

where $h_{0}(X)$ is an Artin motive and $h_{1}(X)$ is a direct summand of $h_{1}(C)$ for some smooth projective curve $C$ (which can be chosen as an iterated hyperplane section of $X)$. This decomposition yields a partial Künneth decomposition for any Weil cohomology $H$ verifying (1.3) (for example, $H$ classical), because it lifts by construction Kleiman's partial Künneth decomposition in [65], which uses (1.3). We also write $h_{>0}(X)$ for $h_{1}(X) \oplus h_{>1}(X)$.

Lemma 2.1. Let $\sim$ be an adequate equivalence relation. We have (in $\mathrm{Ab} \otimes \mathrm{Q})$

$$
\begin{aligned}
\mathcal{M}_{\sim}\left(h_{>0}(Y), h_{0}(X)\right) & =0 \\
\mathcal{M}_{\sim}(\mathbf{1}, \mathbf{1}) & =\mathbf{Z} \text { for any } \sim \\
\mathcal{M}_{\sim}\left(\mathbf{1}, h_{1}(X)\right) & =\operatorname{Alb}_{X}(k) \text { if } \sim=\text { rat } \\
\mathcal{M}_{\sim}\left(\mathbf{1}, h_{>0}(X)\right) & =0 \text { if } \sim \leq \text { alg } \\
\mathcal{M}_{\sim}\left(h_{1}(Y), h_{1}(X)\right) & =\operatorname{Hom}_{k}\left(\operatorname{Alb}_{Y}, \operatorname{Alb}_{X}\right) \text { for any } \sim \\
\mathcal{M}_{\sim}\left(h(Y), h_{1}(X)\right) & =\operatorname{Mor}_{k}\left(Y, \operatorname{Alb}_{X}\right) \text { if } \sim=\text { rat } \\
\mathcal{M}_{\sim}\left(h_{>1}(Y), h_{1}(X)\right) & =0 .
\end{aligned}
$$

where $Y \in \mathbf{S m}^{\text {proj }}$.

Proof. (2.1) is easy and left to the reader. (2.2) is trivial. (2.3) and (2.5) are [82, Th. 4.4 (iii)] and [82, Prop. 4.5] respectively. (2.4) holds because 0 -cycles of degree 0 are algebraically equivalent to 0 .

The morphism (A.4) of Appendix A provides a map (2.6), hence for this item and (2.7), we may assume $\operatorname{dim} X=1, X, Y$ connected and (by a transfer argument) even geometrically connected. Then

$$
\mathcal{M}(h(Y), h(X))=C H^{1}(Y \times X) .
$$

In the decomposition

$$
\mathcal{M}(h(Y), h(X))=\mathcal{M}\left(h(Y), h_{0}(X)\right) \oplus \mathcal{M}\left(h(Y), h_{1}(X)\right) \oplus \mathcal{M}\left(h(Y), h_{2}(X)\right)
$$

the first (resp. third) summand is $C H^{0}(Y)$ (resp. $C H^{1}(Y)$ ), in view of (1.2) and the isomorphisms $h_{0}(X)=\mathbf{1}, h_{2}(X) \simeq \mathbb{L}$. Comparing with Weil's formula [82, Th. 3.9 (i)]

$$
C H^{1}(Y \times X) \simeq C H^{1}(Y) \oplus C H^{1}(X) \oplus \operatorname{Hom}\left(\operatorname{Alb}_{Y}, J(X)\right)
$$


and checking that the idempotents match concludes the proof.

Proposition 2.2 (compare [82, 1.11], [56, Cor. 7.8.10] and [76, Th. 5.2.6]). Let $i \in\{0,1\}$. Write $w_{\leq i} \mathcal{M}$ for the thick subcategory of $\mathcal{M}$ generated by the $h_{j}(X)^{\prime} s$ for $j \leq i$, where $X$ runs through smooth projective irreducible varieties. Then:

(1) The inclusion functor $w_{<i} \mathcal{M} \hookrightarrow \mathcal{M}$ has a left adjoint, denoted by $w_{\leq i}$. We have $w_{\leq 0} h(X)=h_{0}(X)$ and $w_{\leq 1} h(X)=h_{0}(X) \oplus$ $h_{1}(X)$ for $X \in \mathbf{S m}^{\text {proj }}$.

(2) For $M \in \mathcal{M}$, the unit morphism $M \rightarrow w_{\leq i} M$ is split epi, whence a split exact sequence

$$
0 \rightarrow w_{>i} M \rightarrow M \rightarrow w_{\leq i} M \rightarrow 0
$$

natural in $M$, where $w_{>i} M:=\operatorname{Ker}\left(M \rightarrow w_{\leq i} M\right)$. We have $w_{>i} h(X)=h_{>i}(X)$ for $X \in \mathbf{S m}^{\text {proj }}$.

(3) For $X \in \mathbf{S m}^{\text {proj }}$, the objects $h_{i}(X)$ and $h_{>i}(X)(i=0,1)$ are independent of the choices of $C K$ decompositions, and are functorial for the action of correspondences.

Proof. In (1), it suffices to check that the left adjoint is defined on motives of the form $h(X)$, which follows from (2.1) and (2.7). Same reduction for (2); (3) is an obvious consequence of (1) and (2).

\section{The motive of A Surface (REvieW)}

3.1. The transcendental part. Let $S$ be a surface over $k$. In [56], we refined Murre's Chow-Künneth decomposition of $h(S) \in \mathcal{M}$

$$
h(S)=\bigoplus_{i=0}^{4} h_{i}(S)
$$

by splitting the motive $h_{2}(S)$ into

$$
h_{2}(S)=t_{2}(S) \oplus \mathrm{NS}_{S}(1)
$$

where $\mathrm{NS}_{S}$ is the Artin motive associated to the Néron-Severi group of $S_{k_{s}}$. This decomposition was anticipated in [41, end of Section 2].

The motive $t_{2}(S)$ is called the transcendental part of $h(S)$. It controls the Albanese kernel in the sense that

$$
T(S) \simeq \mathcal{M}\left(\mathbf{1}, t_{2}(S)\right)
$$

Moreover, $t_{2}(S)$ is a "birational motive" in the following sense:

$$
\begin{aligned}
& \mathcal{M}\left(M(1), t_{2}(S)\right)=0 \text { for any } M \in \mathcal{M}, \\
& \mathcal{M}\left(h(Y), t_{2}(S)\right) \simeq T\left(S_{k(Y)}\right) \text { for any (connected) } Y \in \mathbf{S m}^{\text {proj }}
\end{aligned}
$$


[56, Cor. 7.8.5]. By Poincaré duality, we also have from (3.3):

$$
\mathcal{M}\left(t_{2}(S), \mathbb{L}\right)=0
$$

See proposition 7.6 for a higher-dimensional version of these facts.

Lemma 3.1. With notation as in Section 2, the projections $h_{>1}(S) \rightarrow$ $h_{2}(S)$ and $h_{2}(S) \rightarrow t_{2}(S)$ do not depend on the choice of the refined $C K$ decomposition of $S$.

Proof. For the first, it suffices to see that

$$
\mathcal{M}\left(h_{4}(S) \oplus h_{3}(S), h_{2}(S)\right)=0,
$$

which follows from (2.1) and (2.7) by Poincaré duality. For the second, it similarly suffices to observe that $\mathcal{M}\left(\mathrm{NS}_{S}(1), t_{2}(S)\right)=0$, which follows from (3.3).

For simplicity, assume $Y$ geometrically connected. If it has a $\mathrm{CK}$ decomposition, we get from (3.4) a decomposition

$$
T\left(S_{k(Y)}\right) / T(S) \simeq \bigoplus_{i=1}^{2 \operatorname{dim} Y} \mathcal{M}\left(h_{i}(Y), t_{2}(S)\right)
$$

which shows how the growth of $T(S)$ under extensions of $k$ is controlled by Chow-Künneth summands. A first application is if $Y$ is a curve $C$ : we get thanks to (3.3):

$$
T\left(S_{k(C)}\right) / T(S) \simeq \mathcal{M}\left(h_{1}(C), t_{2}(S)\right) .
$$

Here is another application:

3.2. Mumford's theorem. In (3.4), take $Y=S^{n}$ for some $n>0$. Then the CK decomposition of $S$ induces a CK decomposition of $S^{n}$. In particular, $h_{2}(S)^{\oplus n}$ is a direct summand of $h\left(S^{n}\right)$, and so is $t_{2}(S)^{\oplus n}$. In view of (3.6), this shows that

$$
\text { End }_{\mathcal{M}}\left(t_{2}(S)\right)^{n} \text { is a direct summand of } T\left(S_{k\left(S^{n}\right)}\right) / T(S)
$$

and (3.6) also gives the equivalences

$$
\begin{aligned}
t_{2}(S) \neq 0 \Longleftrightarrow \operatorname{End}_{\mathcal{M}}\left(t_{2}(S)\right) \neq 0 & \Longleftrightarrow T\left(S_{k(S)}\right) / T(S) \neq 0 \\
& \Longleftrightarrow \operatorname{dim}_{\mathbf{Q}} T\left(S_{\Omega}\right) \geq \frac{1}{2} \operatorname{trdeg}(\Omega / k)
\end{aligned}
$$

if $\Omega$ is an algebraically closed field containing $k$, of transcendence degree $\geq 2$ (possibly infinite). (By [41], one can replace "transcendence degree $\geq 2$ " by "transcendence degree $\geq 1$ " by using (3.7), and this is precisely what we shall refine in Part 2 in terms of the Griffiths group.) 
Suppose $k$ algebraically closed. If $H$ is a classical Weil cohomology with coefficients in the field $F$, recall the orthogonal decomposition

$$
H^{2}(S)=\mathrm{NS}(S) \otimes F \oplus H_{\mathrm{tr}}^{2}(S)
$$

for the Poincaré pairing. Since the homological realisation of $t_{2}(S)$ is $H_{\mathrm{tr}}^{2}(S)$ [56, Prop. 7.2.3], a sufficient condition for $t_{2}(S) \neq 0$ is that $b^{2}>\rho$. This gives a simple motivic proof of Mumford's theorem on the "hugeness" of $T\left(S_{\mathbf{C}}\right)$ if $p_{g}>0$, in Bloch's style [16, App. to Lect. 1].

\section{Grading By COniveau}

The following definition and lemmas use the semi-simplicity of $\mathcal{M}_{\text {num }}$ (Jannsen [46]).

Definition 4.1. Let $S \in \mathcal{M}_{\text {num }}$ be a simple motive. We say that $S$ is primitive if $S(-1)$ is not effective. We say that $M \in \mathcal{M}_{\text {num }}$ is primitive if every simple summand of $M$ is primitive.

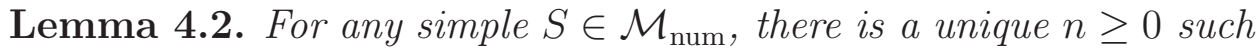
that $S(-n)$ is primitive. We call $n$ the coniveau of $S$ and write it $\nu(S)$.

Proof. Write $S$ as a direct summand of $h(X)$ for some $X \in \mathbf{S m}^{\text {proj }}$. Suppose that $S(-n)$ is effective; then $S$ is also a direct summand of $h\left(X_{n}\right)(n)$ for some $X_{n} \in \mathbf{S m}^{\text {proj }}$. Therefore

$$
0 \neq \mathcal{M}_{\text {num }}\left(h\left(X_{n}\right)(n), h(X)\right)=A_{\text {num }}^{\operatorname{dim} X-n}\left(X_{n} \times X\right)
$$

showing that $\nu(S) \leq \operatorname{dim} X$.

This yields:

Proposition 4.3. For any $M \in \mathcal{M}_{\text {num }}$, there is a unique decomposition

$$
M=\bigoplus_{j \geq 0} M_{j}(j)
$$

with $M_{j}$ primitive. This is the coniveau decomposition of $M$.

Proof. Write $M$ as a direct sum of its isotypical components and group by coniveau.

Let $H$ be a classical Weil cohomology (see $\S 1.6$ ). An object $M \in \mathcal{M}_{H}$ has weight $i$ if $H^{j}(M)=0$ for $j \neq i$. In particular, $i<0 \Rightarrow M=0$. A weight decomposition of $M$ is a (necessarily unique) decomposition

$$
M=\bigoplus_{i \geq 0} M_{i}
$$

with $M_{i}$ of weight $i$. if $M=h(X)$ for some variety $X$, we recover the Künneth decomposition. 
Let $\mathcal{M}_{H}^{*}$ denote the full subcategory of $\mathcal{M}_{H}$ consisting of motives having a weight decomposition. Then for any $M \in \mathcal{M}_{H}^{*}$, the ideal

$$
\operatorname{Ker}\left(\operatorname{End}_{\mathcal{M}_{H}}(M) \rightarrow \operatorname{End}_{\mathcal{M}_{\text {num }}}(M)\right)
$$

is nilpotent [8, Prop. 5]. Hence the decomposition (4.1) for $M_{\text {num }}$ lifts from numerical to homological equivalence, uniquely up to isomorphism.

Lemma 4.4. Let $M \in \mathcal{M}_{H}^{*}$ be of weight $i$. Then, in a lift of (4.1) from $\mathcal{M}_{\text {num }}$ to $\mathcal{M}_{H}$, the motive $M_{j}$ is

- 0 if $i-2 j<0$;

- an Artin motive if $i-2 j=0$;

- of the form $h_{1}(A)$ for some abelian variety $A$ if $i-2 j=1$.

Proof. In $\mathcal{M}_{H}^{*}, M_{j}$ is of weight $i-2 j$, hence of the said form. Indeed, this is trivial for $i-2 j \leq 0$. For $i-2 j=1$, writing $M_{j}$ as a direct summand of $h(Y)$ for some (not necessarily connected) $Y \in \mathbf{S m}^{\text {proj }}$, we find that $M_{j}$ is a direct summand of $h_{1}(Y)$, and conclude by (2.5) and Poincaré complete reducibiity.

\section{The Motive of CERTAin 3-Folds}

Until Section 8, we let $T$ be a geometrically connected 3-fold admitting a CK decomposition

$$
h(T)=\bigoplus_{i=0}^{6} h_{i}(T) \in \mathcal{M} .
$$

Examples include $T=C \times S$, smooth complete intersections in $\mathbf{P}^{N}$ and abelian varieties $[57, \S 3.4]$.

We now "refine" (5.1) in the style of [56, Th. 7.7 .3 (iii)]:

Applying (4.1) to $h_{i}(T)$ in $\mathcal{M}_{\text {num }}$, we may write it

$$
h_{i}(T)=\bigoplus_{j \geq 0} h_{i, j}(T)(j) .
$$

Lemma 5.1. The decomposition (5.2) lifts from numerical to rational equivalence. The motive $h_{2,1}(T)$ is an Artin motive and $h_{3,1}(T)$ is of the form $h_{1}\left(J^{2}\right)$ for some (isogeny class of) abelian variety $J^{2}=J^{2}(T)$.

Proof. By Poincaré duality, we have $h_{6-i}(T) \simeq h_{i}(T)^{*}(3)$ for $i \leq 3$, hence we may assume $i \leq 3$. Then the only nontrivial decompositions are for $i=2,3$ :

$$
\begin{aligned}
& h_{2}(T)=t_{2}(T) \oplus h_{2,1}(T)(1) \\
& h_{3}(T)=t_{3}(T) \oplus h_{3,1}(T)(1)
\end{aligned}
$$


where we write $t_{i}(T):=h_{i, 0}(T)$ for simplicity; the claims on $h_{2,1}(T)$ and $h_{3,1}(T)$ follow from Lemma 4.4 .

Let $p \in \mathcal{M}_{\text {num }}\left(h_{i}(T), h_{i, 1}(T)(1)\right), q \in \mathcal{M}_{\text {num }}\left(h_{i, 1}(T)(1), h_{i}(T)\right)$ be the two morphisms corresponding to these decompositions. We have $p q=1$. Lift $p$ and $q$ to morphisms $\tilde{p}$ and $\tilde{q}$ in $\mathcal{M}$. Since

$$
\operatorname{End}_{\mathcal{M}}\left(h_{i, j}(T)\right) \stackrel{\sim}{\rightarrow} \operatorname{End}_{\mathcal{M}_{\text {num }}}\left(h_{i, j}(T)\right)
$$

by Lemma 4.4 and $(2.2)$, (2.5), we still have $\tilde{p} \tilde{q}=1$. Then $\tilde{q} \tilde{p} \in$ $\operatorname{End}_{\mathcal{M}}\left(h_{i}(T)\right)$ is idempotent, hence the promised decomposition.

\section{The SUMmands OF $h_{*}(T)$}

One should compare some computations in this section and the next one with those of Gorchinskiy-Guletskir [39, $\S \S 4,5]$, although we don't use cohomology here except in the proof of Proposition 6.4.

6.1. The Artin summands. The Artin motives $h_{2 n, n}(T)$ are easy to compute:

Lemma 6.1. For $n \in[0,3], h_{2 n, n}(T)$ is the Artin motive associated to the Galois module $A_{n}^{\text {num }}\left(T_{k_{s}}\right)$, where $k_{s}$ is a separable clusure of $k$.

Proof. For any adequate equivalence relation, write

$$
A_{n}^{\sim}(T)=\mathcal{M}_{\sim}\left(\mathbb{L}^{n}, h(T)\right)=\bigoplus_{i, j} \mathcal{M}_{\sim}\left(\mathbb{L}^{n}, h_{i, j}(T)(j)\right)
$$

For $\sim=$ num, all terms with $i \neq 2 n$ are 0 for weight reasons. This leaves

$$
\begin{aligned}
A_{n}^{\text {num }}(T)=\mathcal{M}_{\text {num }}\left(\mathbb{L}^{n}, h_{2 n}(T)\right) & \\
& =\mathcal{M}_{\text {num }}\left(\mathbb{L}^{n}, h_{2 n, n}(T)(n)\right)=\mathcal{M}_{\text {num }}\left(\mathbf{1}, h_{2 n, n}(T)\right)
\end{aligned}
$$

by definition of $h_{2 n, n}(T)$ and Schur's lemma. This computation over all finite separable extensions of $k$ yields the lemma.

6.2. The abelian summand. Here we study the abelian variety $J^{2}$ introduced in Lemma 5.1. We first note:

Lemma 6.2. $J^{2}$ contains $P_{T} \approx \mathrm{Alb}_{T}$ as a direct summand (up to isogeny).

Proof. Let $L \in C H^{1}(T)$ be the class of a smooth hyperplane section. By Murre and Scholl [73, 82], intersection product with $L^{3-i}$ induces isomorphisms

$$
h_{6-i}(T) \stackrel{\sim}{\rightarrow} h_{i}(T)(3-i)
$$


for $i=0,1$. For $i=1$, (6.2) factors through $h_{3}(T)(1)$, which implies that $h_{1}(T)$ is a direct summand of $h_{3,1}(T)$ in $\mathcal{M}_{\text {num }}$.

We now give two universal properties of $J^{2}$ in terms of numerical equivalence. For this, note that multiplication by integers $n$ on any abelian variety $A$ acts on $h_{i}(A)$ by the homothety of scale $n^{i}$ [33]. Thus the inclusion $h_{1}\left(J^{2}\right)(1) \hookrightarrow h_{3}(T)$ (resp. the projection $h_{3}(T) \rightarrow$ $\left.h_{1}\left(J^{2}\right)(1)\right)$ obtained from (5.4) defines a canonical element

$$
P^{2} \in A_{\text {num }}^{2}\left(J^{2} \times T\right)_{\mathbf{Q}}^{[1]} \quad\left(\text { resp. } P_{2} \in A_{2}^{\text {num }}\left(T \times J^{2}\right)_{\mathbf{Q}}^{[1]}\right)
$$

(Poincaré classes), where the superscript ${ }^{[1]}$ means the eigensubspace where multiplication by $m$ on $J^{2}$ acts by the homothety of scale $m$.

Lemma 6.3. a) The classes $P^{2}$ and $P_{2}$ verify

$$
\left\langle P^{2}, P_{2}\right\rangle=1
$$

for the intersection pairing.

b) For any abelian variety $A$ and any $\alpha \in A_{\text {num }}^{2}(A \times T)_{\mathbf{Q}}^{[1]}$, there is a unique (isogeny class of) morphism $u: A \rightarrow J^{2}$ such that $u^{*} P^{2}=\alpha$.

c) For any abelian variety $A$ and any $\alpha \in A_{2}^{\text {num }}(T \times A)_{\mathbf{Q}}^{[1]}$, there is a unique (isogeny class of) morphism $u: J^{2} \rightarrow A$ such that $u_{*} P_{2}=\alpha$.

Proof. a) follows from the fact that the composition

$$
h_{1}\left(J^{2}\right)(1) \stackrel{P^{2}}{\longrightarrow} h_{3}(T) \stackrel{P_{2}}{\longrightarrow} h_{1}\left(J^{2}\right)(1)
$$

is the identity. Let us prove b) (the proof of c) is dual). Such an $\alpha$ corresponds to a morphism $h_{1}(A)(1) \rightarrow h(T)$ in $\mathcal{M}_{\text {num }}$, which factors uniquely through $P^{2}$ by uniqueness of the refined Künneth decomposition.

Next, we want to compare $J^{2}$ with Murre's algebraic representative $\mathrm{Ab}^{2}$ [72]; by [1, Th. 4.4 and Rk. 4.5], it is defined over $k$ provided $k$ is perfect. Since the sequel will only be used in Remark 7.11, we only sketch some arguments.

From the split epimorphism $h(T) \rightarrow h_{1}\left(J^{2}\right)(1)$ of Lemma 5.1, we get a split epimorphism in $\mathbf{A} \mathbf{b} \otimes \mathbf{Q}$

$$
\mathcal{M}(h(X)(1), h(T)) \rightarrow \mathcal{M}\left(h(X)(1), h_{1}\left(J^{2}\right)(1)\right)
$$

for any $X \in \mathbf{S m}^{\text {proj }}$. Using (2.6) and translating to $\mathbf{A b}$, this amounts (after choosing an abelian variety $\tilde{J}^{2}$ in the isogeny class $J^{2}$ ) to an honest homomorphism $\varphi(X): C H^{2}(X \times T) \rightarrow \widetilde{J}^{2}(X)$, natural for the action of correspondences, and a natural homomorphism $\sigma(X)$ in the opposite direction such that $\varphi(X) \sigma(X)=m$ for an integer $m>0$ 
independent of $X$. In particular, when $k$ is algebraically closed we get an epimorphism (for $X=\operatorname{Spec} k$ ):

$$
\varphi(k): C H^{2}(T) \rightarrow \tilde{J}^{2}(k) .
$$

The naturality of $\varphi$ then shows that, for any $Y \in C H^{2}(X \times T)$, the composition

$$
X(k) \stackrel{\theta}{\rightarrow} C H_{0}(X) \stackrel{Y_{*}}{\rightarrow} C H^{2}(T) \stackrel{\varphi(k)}{\longrightarrow} \tilde{J}^{2}(k)
$$

(where $\theta(x)=[x]$ ) is induced by $\varphi(X)(Y)$. Hence $\varphi$ is regular, and so is its restriction to $C H^{2}(T)$ alg. Since $\mathcal{M}\left(\mathbb{L}, h_{1}\left(J^{2}\right)(1)\right)$ consists of algebraically trivial cycles $(2.4), \sigma($ Spec $k)$ lands into $C H^{2}(T)_{\text {alg, so }}$ that $\varphi_{\mid C H^{2}(T)_{\text {alg }}}$ is still surjective. Coming back to the general case, this and the universal property of $\mathrm{Ab}^{2}$ yield an epimorphism

$$
v: \mathrm{Ab}_{\bar{k}}^{2} \longrightarrow \tilde{J}_{\bar{k}}^{2}
$$

where $\bar{k}$ is an algebraic closure of $k$, that we assume perfect.

Proposition 6.4. The morphism $v$ is an isogeny defined over $k$.

Sketch. ${ }^{2}$ Let $A=\mathrm{Ab}^{2}$. By [72, Cor. 1.6.3] and [1, Lemma 4.9], there exists a correspondence $Y \in C H^{2}(A \times T)$ and a positive integer $r$ such that we have $\psi \circ Y_{*} \circ \theta_{0}=r 1_{A(\bar{k})}$, where $\psi: C H^{2}\left(T_{\bar{k}}\right)_{\text {alg }} \rightarrow A(\bar{k})$ is the universal regular homomorphism and $\theta_{0}(a)=[a]-[0]$.

We claim that we can choose $Y$ such that its image in $A_{\text {num }}^{2}(A \times T)_{\mathbf{Q}}$ lies in $A_{\text {num }}^{2}(A \times T)_{\mathbf{Q}}^{[1]}$. Granting this claim, the universal property of Lemma $6.3 \mathrm{~b})$ provides a $k$-homomorphism $u: A \rightarrow \tilde{J}^{2}$ such that the map $A(\bar{k}) \stackrel{r}{\rightarrow} A(\bar{k})$ factors through $u(\bar{k})$. But the construction of the natural transformation $\varphi$ then shows that $u(\bar{k})=r v(\bar{k})$; thus $u_{\bar{k}}=r v$, and $v$ is an isogeny defined over $k$.

To prove the claim, we first note that since $A$ has a CK decomposition [33], we may write any $Z \in C H^{2}(A \times T)=\mathcal{M}(h(A)(1), h(T))$ as $Z=\sum Z^{i}$ where $Z^{i} \in \mathcal{M}\left(h_{i}(A)(1), h(T)\right)$. It therefore suffices to show that, if $Z^{1}=0$, then $\psi \circ Z_{*} \circ \theta_{0}=0$. (Indeed, we then have $\left.\psi \circ Y_{*} \circ \theta_{0}=\psi \circ Y_{*}^{1} \circ \theta_{0}.\right)$

For this, we use Bloch's maps $\lambda_{X}^{i}: C H^{i}(X)\{l\} \rightarrow H_{\text {êt }}^{2 i-1}\left(X, \mathbf{Q}_{l} / \mathbf{Z}_{l}(i)\right)$ from [15], with $l \neq$ char $k$ : by loc. cit., Prop. 3.5, they are compatible with the action of correspondences. (This could be seen more easily by interpreting the $\lambda^{i}$ in a modern way in terms of étale motivic cohomology via [53, Prop. 4.17].) Let $d=\operatorname{dim} A$. By hypothesis, $Z: H_{\text {ét }}^{2 d-1}\left(A, \mathbf{Q}_{l}(d)\right) \rightarrow H_{\text {ét }}^{3}\left(T, \mathbf{Q}_{l}(2)\right)$ is 0 as it acts via $Z^{1}$, hence $Z: H_{\text {êt }}^{2 d-1}\left(A, \mathbf{Q}_{l} / \mathbf{Z}_{l}(d)\right) \rightarrow H_{\text {êt }}^{3}\left(T, \mathbf{Q}_{l} / \mathbf{Z}_{l}(2)\right)$ has finite image. Since $\lambda_{T}^{2}$ is injective [72, Prop. 9.2], we find that $\psi \circ Z_{*} \circ \theta_{0}$ has finite image in $A(k)\{l\}$. Since $\psi$ is regular, $\psi \circ Z_{*} \circ \theta_{0}$ is induced by an endomorphism of $A$, which must be trivial.

\footnotetext{
${ }^{2}$ The sketch consists of neglecting to keep track of some denominators in $\S \S 2-3$.
} 
Remark 6.5. The cycle $Y$ appearing in the proof of Proposition 6.4 is close to Voisin's "universal" codimension 2 cycle ([97, Def. 1.5], see also [25, Déf. 5.3]). The difference is that, in loc. cit., the integer $r$ is required to be equal to 1 .

\section{7. $t_{3}(T)$ AND THE GRIFFITHS GROUP}

In this section, we do two related things:

- Study the uniqueness of the lifts from Lemma 5.1: see Corollary 7.4 and Lemma 7.5. (Recall that the corresponding lift for a surface is unique [56].)

- Relate the Griffiths group of $T$ to the motive $t_{3}(T)$ : see Theorem 7.7 and Conjecture 7.8 .

7.1. A Chow-Lefschetz condition. To go further, we need:

Hypothesis 7.1. There exists a smooth hyperplane section $\Sigma \subset T$ such that (6.2) is an isomorphism also for $i=2$.

This is true in the same cases as in $\S 5$ :

Proposition 7.2. Hypothesis 7.1 holds if $T$ is an abelian variety, a complete intersection in $\mathbf{P}^{N}$ or for $T=C \times S$ if $\Sigma$ is defined as in [65, Prop. 1.4.6 (ii)].

Proof. The first case follows from [68], the second one is trivial and the last one is proven in Theorem B.1.

\subsection{Uniqueness of (5.3).}

Proposition 7.3. Let $\Sigma \stackrel{i}{\rightarrow} T$ be a smooth hyperplane section as in Hypothesis 7.1. Then the morphism $i_{*}: h(\Sigma) \rightarrow h(T)$ induces a split surjection $t_{2}(\Sigma) \longrightarrow t_{2}(T)$.

Proof. The isomorphism (6.2) factors as

$$
h_{4}(T) \stackrel{i^{*}}{\rightarrow} h_{2}(\Sigma)(2) \stackrel{i_{*}}{\rightarrow} h_{2}(T)(2),
$$

showing that $i_{*}: h_{2}(\Sigma) \rightarrow h_{2}(T)$ is split surjective. Let $\lambda: h_{2}(T) \rightarrow$ $h_{2}(\Sigma)$ be a right inverse. Using the decompositions (3.1) and (5.3), write $i_{*}(\operatorname{resp} . \lambda)$ as a $2 \times 2$ matrix

$$
i_{*}=\left(\begin{array}{ll}
a & b \\
c & d
\end{array}\right), \quad \lambda=\left(\begin{array}{ll}
a^{\prime} & b^{\prime} \\
c^{\prime} & d^{\prime}
\end{array}\right) .
$$

Then $b^{\prime}=c=0$ by (3.3) and (3.5), and $b, c^{\prime}$ are numerically equivalent to 0 by Schur's lemma, hence $c^{\prime} b=0$ by $(2.2)$. From $i_{*} \lambda=1$, 
we get $a a^{\prime}=1-b c^{\prime}$ with $\left(b c^{\prime}\right)^{2}=b c^{\prime} b c^{\prime}=0$; thus $a a^{\prime}\left(1+b c^{\prime}\right)=1$ and $a: t_{2}(\Sigma) \rightarrow t_{2}(T)$ has a right inverse.

Corollary 7.4. Under Hypothesis 7.1, the lift of (5.3) given by Lemma 5.1 is unique, up to unique isomorphism.

Proof. Proposition 7.3, (3.3) and (3.5) imply that there are no nonzero homomorphisms between the two summands.

\subsection{Uniqueness of (5.4).}

Lemma 7.5. Assume that $h_{3}(T)$ is finite-dimensional in the sense of Kimura [64], e.g. T is of abelian type. Then the lift of (5.4) given by Lemma 5.1 is unique, up to possibly non-unique isomorphism.

Proof. This follows from Kimura's nilpotence theorem [64, Prop. 7.5].

7.4. Birational properties of $t_{3}(T)$. The next proposition uses Voevodsky's triangulated category of motives $\mathbf{D M}_{\mathrm{gm}}^{\mathrm{eff}}[94]$ (with Q coefficients). Recall that there is a canonical fully faithful functor [12, Cor. $6.7 .3]$

$$
\Phi: \mathcal{M} \rightarrow \mathrm{DM}_{\mathrm{gm}}^{\mathrm{eff}}
$$

Proposition 7.6. We have $\underline{\operatorname{Hom}}\left(\mathbf{Z}(2), \Phi\left(t_{3}(T)\right)\right)=0$ in $\mathbf{D} \mathbf{M}_{\mathrm{gm}}^{\mathrm{eff}}$. In particular,

$$
\mathcal{M}\left(M(2), t_{3}(T)\right)=0
$$

for any $M \in \mathcal{M}$ and

$$
\mathcal{M}(k)\left(h(Y)(1), t_{3}(T)\right) \simeq \mathcal{M}(k(Y))\left(\mathbb{L}, t_{3}(T)_{k(Y)}\right)
$$

for any connected $Y \in \mathbf{S m}^{\mathrm{proj}}$.

Proof. We proceed as in the proof of [56, Th. 7.8.4 b)]. So we reduce to showing that

$$
\mathbf{D M}_{\mathrm{gm}}^{\mathrm{eff}}(K)\left(\mathbf{Z}(2)[4], \Phi\left(t_{3}\left(T_{K}\right)[i]\right)\right)=0
$$

for any function field $K / k$ and any $i \in \mathbf{Z}$. By Poincaré duality, this group is a direct summand (in $\mathbf{A} \mathbf{b} \otimes \mathbf{Q}$ ) of

$$
\mathbf{D M}_{\mathrm{gm}}^{\mathrm{eff}}(K)\left(\mathbf{Z}(2)[4], M\left(T_{K}\right)[i]\right) \simeq H_{\mathrm{Nis}}^{i+2}\left(T_{K}, \mathbf{Z}(1)\right) \simeq H_{\mathrm{Nis}}^{i+1}\left(T_{K}, \mathbb{G}_{m}\right) .
$$

Recall that this group is 0 for $i \neq 0,-1$. For $i=0$, it corresponds to the summand $(3,0)$ in the decomposition

$$
\operatorname{Pic}\left(T_{K}\right)=C H_{2}\left(T_{K}\right)=\bigoplus_{(i, j)} \mathcal{M}(K)\left(\mathbb{L}^{2}, h_{i, j}(T)(j)\right) .
$$


We find $\operatorname{NS}\left(T_{K}\right)$ and $\operatorname{Pic}^{0}\left(T_{K}\right)$ respectively as the summands $(4,2)$ and $(5,0)$ (the latter because $\left.h_{5}(T) \simeq h_{1}(T)(2)\right)$. Thus all other summands are 0 . For $i=-1$ we reason similarly.

This and the full faithfulness of $\Phi$ immediately implies (7.2). To prove (7.3), we note that (7.2) implies that the functor $\mathcal{M} \ni M \mapsto$ $\mathcal{M}\left(M(1), t_{3}(T)\right)$ factors through the category $\mathcal{M}^{\circ}$ of birational Chow motives of [57]; we can then use the adjunction of [54, Th. 6.6].

\subsection{The Griffiths group.}

Theorem 7.7. Under Hypothesis 7.1, we have canonical isomorphisms

$$
\operatorname{Griff}(T) \simeq \mathcal{M}_{\mathrm{alg}}\left(\mathbb{L}, t_{3}(T)\right) \simeq \mathcal{M}_{\mathrm{alg}}\left(t_{3}(T), \mathbb{L}^{2}\right)
$$

for any lift of (5.4) to $\mathcal{M}_{\mathrm{alg}}$.

Proof. In (6.1), take $n=1$. We first examine which terms vanish for any $\sim$. This is the case of the summands for $i=0,1$. For $i=2$, we have

$$
\mathcal{M}_{\sim}\left(\mathbb{L}, h_{2}(T)\right)=\mathcal{M}_{\sim}\left(\mathbb{L}, t_{2}(T)\right) \oplus \mathcal{M}_{\sim}\left(\mathbb{L}, h_{2,1}(T)(1)\right)=A_{1}^{\mathrm{num}}(T)
$$

by (3.3) and Proposition 7.3.

For $i=3$, we have

$$
\mathcal{M}_{\sim}\left(\mathbb{L}, h_{3,1}(T)(1)\right)=\mathcal{M}_{\sim}\left(\mathbf{1}, h_{1}\left(J^{2}\right)\right) .
$$

For $i=4$, we have

$$
\begin{aligned}
\mathcal{M}_{\sim}\left(\mathbb{L}, h_{4}(T)\right) \stackrel{\sim}{\rightarrow} & \mathcal{M}_{\sim}\left(\mathbb{L}, h_{2}(T)(1)\right) \\
& =\mathcal{M}_{\sim}\left(\mathbf{1}, t_{2}(T) \oplus A_{1}^{\text {num }}(T)(1)\right)=\mathcal{M}_{\sim}\left(\mathbf{1}, t_{2}(T)\right) ;
\end{aligned}
$$

for $\sim=$ rat, the last group is a direct summand of $T(\Sigma)$ as in Proposition 7.3.

Finally, for $i=5,6$ we find

$$
\begin{array}{r}
\mathcal{M}_{\sim}\left(\mathbb{L}, h_{5}(T)\right) \simeq \mathcal{M}_{\sim}\left(\mathbf{1}, h_{1}(T)(1)\right)=0 \\
\mathcal{M}_{\sim}\left(\mathbb{L}, h_{6}(T)\right) \simeq \mathcal{M}_{\sim}\left(\mathbb{L}, \mathbb{L}^{3}\right)=0 .
\end{array}
$$

Thus, for $\sim=$ alg, the only nonzero terms are for $(i, j)=(2,1),(3,0)$; this completes the proof of Theorem 7.7, except for the second isomorphism. To prove it, we note that Poincaré duality and the uniqueness of the coniveau decomposition (4.1) yield an isomorphism in $\mathcal{M}_{\text {num }}$ :

$$
t_{3}(T) \simeq t_{3}(T)^{*}(3) .
$$

Since the first isomorphism of (7.4) is valid for any lift of $t_{3}(T)$ to $\mathcal{M}_{\text {alg }}$, we get the second one by replacing $t_{3}(T)$ with $t_{3}(T)^{*}(3)$. (This argument avoids the uniqueness issue from Lemma 7.5.) 


\subsection{A conjecture.}

Conjecture 7.8. The map $\mathcal{M}\left(\mathbb{L}, t_{3}(T)\right) \rightarrow \mathcal{M}_{\text {alg }}\left(\mathbb{L}, t_{3}(T)\right)$ is bijective.

Proposition 7.9. Conjecture 7.8 follows from the Bloch-BeilinsonMurre (BBM) conjectures (see [47]).

To prove this proposition, we need a lemma:

Lemma 7.10 (Weil-Bloch trick). Let $F: \mathcal{M} \rightarrow \mathbf{A b} \otimes \mathbf{Q}$ be a contravariant additive functor. Then $F$ factors through $\mathcal{M}_{\text {alg }}$ if and only if $F\left(1_{M} \otimes \alpha\right)=0$ for any $M \in \mathcal{M}$, any $k$-curve $\Gamma$ and any $\alpha \in \operatorname{Pic}^{0}(\Gamma)=$ $\mathcal{M}\left(\mathbf{1}, h_{1}(\Gamma)\right)$. We may restrict to $M=h(Y)$ for $Y \in \mathbf{S m}^{\text {proj }}$.

Apply this lemma to $F(M)=\mathcal{M}\left(M(1), t_{3}(T)\right)$. We shall use [47, Prop. 5.8], which Jannsen shows to be a consequence of the BBM conjectures that we now assume. If $M=h(Y)$, then for any $k$-curve $\Gamma$

$$
\begin{aligned}
& \mathcal{M}(k)\left(h(Y) \otimes h_{1}(\Gamma)(1), t_{3}(T)\right) \simeq \mathcal{M}(k(Y))\left(h_{1}(\Gamma)(1), t_{3}(T)\right) \\
& \stackrel{\sim}{\rightarrow} \mathcal{M}_{\text {num }}(k(Y))\left(h_{1}(\Gamma)(1), t_{3}(T)\right) \stackrel{\sim}{\sim} \mathcal{M}_{\text {num }}(k)\left(h_{1}(\Gamma)(1), t_{3}(T)\right)=0 .
\end{aligned}
$$

Here the first isomorphism is (7.3), the second one is a special case of [47, Prop. 5.8], the third one is given by the full faithfulness of $\mathcal{M}_{\text {num }}(k) \rightarrow \mathcal{M}_{\text {num }}(k(Y))$ [54, Prop. 5.5], and the last vanishing is by Schur's lemma and by definition of $t_{3}(T)$. Hence $F$ verifies the condition of Lemma 7.10.

Remark 7.11. Conjecture 7.8 is striking, as it predicts in view of (7.4) that $\operatorname{Griff}(T)$ is (up to isogeny) cut off $C H^{2}(T)$ by an idempotent self-correspondence of $T$, namely the one defining $t_{3}(T)$. According to Beilinson's vision, it also predicts an isomorphism

$$
\operatorname{Griff}(T) \simeq \operatorname{Ext}_{\mathcal{M M}}^{1}\left(\mathbb{L}, t_{3}(T)\right)
$$

where $\mathcal{M M}$ is the hypothetical abelian category of mixed motives, see $[47,(2.3)]$.

It is also related to a conjecture of Nori [48, p. 227]: codimension 2 cycles in the kernel of the Abel-Jacobi map are algebraically equivalent to 0. Jannsen proves in loc. cit., Th. 6.1 that this holds under the $\mathrm{BBM}$ conjectures and the standard conjecture B.

By the proof of Theorem 7.7, the only summands of $C H^{2}(T)$ which contain algebraically trivial cycles are $\mathcal{M}\left(\mathbb{L}, h_{3,1}(T)(1)\right)=J^{2}(k)$, $\mathcal{M}\left(\mathbb{L}, h_{4}(T)\right)=\mathcal{M}\left(\mathbf{1}, t_{2}(T)\right)$ and $\mathcal{M}\left(\mathbb{L}, t_{3}(T)\right)$. The first two are fully algebraically trivial (2.4). On the other hand, the Abel-Jacobi map is injective on $J^{2}(k)$ by Proposition 6.4 and [72, Th. 1.11.1]. Thus Conjecture 7.8 refines Nori's conjecture as follows, in the case of a 3 -fold $T$ verifying Hypothesis 7.3: 
The kernel of the Abel-Jacobi map on $C H^{2}(T)$ equals $\mathcal{M}_{\sim}\left(\mathbb{L}, h_{4}(T)\right)=\mathcal{M}_{\sim}\left(\mathbf{1}, t_{2}(T)\right)$.

By Proposition 7.9 and [48, Th. 6.1], this statement follows from the BBM conjectures.

In higher dimensions, nothing new is expected to happen: one easily sees that the BBM conjectures plus the standard conjecture B imply that $C H^{2}(X)_{\text {hom }} \rightarrow C H^{2}(T)_{\text {hom }}$ is split injective in $\mathbf{A b} \otimes \mathbf{Q}$ if $T$ is a 3-dimensional general successive hyperplane section of a smooth projective variety $X$.

\section{THE CASE $T=C \times S$}

We now assume that $T=C \times S$, where $C$ is a curve and $S$ is a surface; since $T$ is geometrically connected, so are $C$ and $S$.

\subsection{A reformulation of Theorem 7.7 .}

Proposition 8.1. There are canonical isomorphisms

$$
\mathcal{M}_{\mathrm{alg}}\left(h_{1}(C), t_{2}(S)\right) \simeq \mathcal{M}_{\mathrm{alg}}\left(\mathbb{L}, t_{3}(C \times S)\right) \simeq \operatorname{Griff}(C \times S) .
$$

Proof. In view of Proposition 7.2, the second isomorphism is Theorem 7.7. Let us prove the first. By duality, we may write

$$
\mathcal{M}_{\text {alg }}\left(h_{1}(C), t_{2}(S)\right)=\mathcal{M}_{\text {alg }}\left(\mathbb{L}, h_{1}(C) \otimes t_{2}(S)\right) .
$$

The Künneth isomorphism in $\mathcal{M}$

$$
h_{3}(C \times S) \simeq \bigoplus_{i=0}^{2} h_{i}(C) \otimes h_{3-i}(S)
$$

gets converted via (3.1), (5.3) and (6.2) into an isomorphism

$$
\begin{aligned}
& t_{3}(C \times S) \oplus h_{1}\left(J^{2}\right)(1) \\
& \quad \simeq h_{1}(S)(1) \oplus h_{1}(C) \otimes \mathrm{NS}_{S}(1) \oplus h_{1}(C) \otimes t_{2}(S) \oplus h_{1}(S)(1) .
\end{aligned}
$$

Working modulo numerical equivalence, we may write

$$
h_{1}(C) \otimes t_{2}(S) \simeq t_{3}^{\prime}(C \times S) \oplus M(1)
$$

by Proposition 4.3; proceeding as in Lemma 4.4, we see that $M=h_{1}(B)$ for some abelian variety $B$.

Proceeding as in the proof of Lemma 5.1, we may lift (8.3) to a decomposition in $\mathcal{M}$. Since $\mathcal{M}_{\text {alg }}\left(\mathbf{1}, h_{1}(A)\right)=0$ for any abelian variety $A$ by (2.4), (8.2) and (8.3) together yield (8.1).

Corollary 8.2. There is a natural action of End $J(C)^{\text {op }} \times$ End $t_{2}(S)$ on $\operatorname{Griff}(C \times S)$. 
Corollary 8.3. We take the same notation as in the proof of Proposition 8.1.

a) There is an isogeny

$$
J^{2} \approx B \times\left(\mathrm{Alb}_{S}\right)^{2} \times \mathrm{Alb}_{C} \otimes \mathrm{NS}_{S} .
$$

b) If $t_{2}(S)$ is finite dimensional (equivalently, if $h(S)$ is finite-dimensional), the Chow motives $t_{3}(C \times S)$ and $t_{3}^{\prime}(C \times S)$ are isomorphic.

Proof. Consider (8.2) modulo numerical equivalence, and insert the value of $h_{1}(C) \otimes t_{2}(S)$ given by (8.3). Taking isotypic components, we get two isomorphisms in $\mathcal{M}_{\text {num }}$ :

$$
\begin{gathered}
t_{3}(C \times S) \simeq t_{3}^{\prime}(C \times S) \\
h_{1}\left(J^{2}\right) \simeq h_{1}(B) \oplus 2 h_{1}(S) \oplus h_{1}(C) \otimes \mathrm{NS}_{S} .
\end{gathered}
$$

The isogeny of a) follows from (8.5). If $t_{2}(S)$ is finite dimensional, so are all terms in (8.2) and (8.3). In particular, the Chow motives $t_{3}(C \times S)$ and $t_{3}^{\prime}(C \times S)$ are finite-dimensional, and b) follows from (8.4) and Kimura's nilpotence theorem [64, Prop. 7.5].

Remarks 8.4. a) Without assuming the finite-dimensionality of $t_{2}(S)$, one gets an isomorphism in $\mathcal{M}$

$$
h_{3}(C \times S) \simeq t_{3}(C \times S) \oplus h_{1}\left(J^{2}\right)(1) \simeq t_{3}^{\prime}(C \times S) \oplus h_{1}\left(J^{2}\right)(1)
$$

but it is not clear whether one can cancel the summand $h_{1}\left(J^{2}\right)(1)$ unconditionally.

b) Assuming Conjecture 7.8, the isomorphisms (8.3) and (3.7) yield an isomorphism in $\mathbf{A b} \otimes \mathbf{Q}$

$$
T\left(S_{k(C)}\right) / T(S) \simeq B(k) \oplus \operatorname{Griff}(C \times S) .
$$

Since $\operatorname{Griff}(C \times S)$ is invariant under algebraically closed extensions, this suggests that $E \mapsto T\left(S_{E(C)}\right) / T\left(S_{E}\right)$ is representable by a $k$-group scheme whose identity component is an abelian variety. Can one prove this a priori?

8.2. Curves mapping to surfaces. Let $\psi \in \mathcal{Z}_{1}(C \times S)$ be a correspondence. It induces a morphism in $\mathcal{M}$

$$
\psi_{*}: h(C) \rightarrow h(S) .
$$

Choose a CK decomposition $\left(\pi_{i}^{C}\right)$ of $C$ and a refined CK decomposition $\left(\pi_{i}^{S}\right)$ of $S$, with $\pi_{2}^{S}=\pi_{2}^{\text {alg }}(S)+\pi_{2}^{\operatorname{tr}}(S)$ : we get a composite morphism

$$
\psi_{\#}: h_{1}(C) \longleftrightarrow h(C) \stackrel{\psi_{*}}{\longrightarrow} h(S) \longrightarrow t_{2}(S)
$$


hence, by Proposition 8.1, a class $\left[\psi_{\#}\right]$ in $\operatorname{Griff}(C \times S)$. This class depends on the choice of the $\mathrm{CK}$ decompositions; note however that, thanks to (2.1), it does not depend on the choice of the 0-cycles on $C$ and $S$ used to define $\pi_{0}^{C}$ and $\pi_{0}^{S}$. In Lemma $8.5 \mathrm{~b}$ ) below, we show that a slight variant of $\psi_{\#}$ does not depend on any choice. The main purpose of Part 2 will be to study the non-vanishing of $\left[\psi_{\#}\right]$, in special circumstances.

We want to compare (8.6) with a canonical morphism defined below (8.10).

Thanks to Proposition $2.2(2), \psi_{*}$ induces a commutative diagram of split exact sequences in $\mathcal{M}$, independent of the choice of the CK decompositions (see Section 2 for the notation):

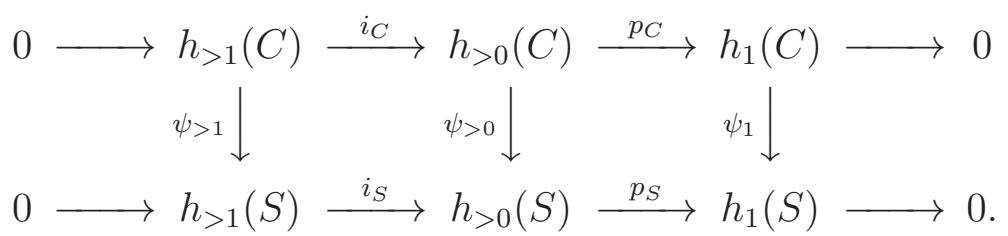

Let $\psi^{*}: h(S) \rightarrow h(C)(1)$ be the transpose of $\psi$. The composition

$$
\mathbb{L}=h_{2}(C)=h_{>1}(C) \stackrel{\psi_{>1}}{\longrightarrow} h_{>1}(S) \stackrel{\psi^{*}}{\rightarrow} h(C)(1) \rightarrow h_{0}(C)(1)=\mathbb{L}
$$

is multiplication by the self-intersection number $[C]^{2}$, where $[C]=$ $\psi_{*} 1 \in \operatorname{Pic}(S)$ with 1 the canonical generator of $C H^{0}(C)$. It is an isomorphism if $[C]^{2} \neq 0$, which we now assume. (This hypothesis is verified if $[C]$ is ample.) Then we can define $h_{>1}(S) / h_{>1}(C)=$ Coker $\psi_{>1}$. On the other hand, by (2.5) we can also define

$$
h_{1}(\psi)=\operatorname{Ker} \psi_{1}
$$

which does not depend on the choice of the CK decompositions. The snake lemma then yields a morphism $h_{1}(\psi) \rightarrow h_{>1}(S) / h_{2}(C)$, that we compose with the projection $h_{>1}(S) / h_{2}(C) \rightarrow h_{2}(S) / h_{2}(C)$ to obtain

$$
\tilde{\psi}: h_{1}(\psi) \rightarrow h_{2}(S) / h_{2}(C) \text {. }
$$

In view of $(3.4)$, the projection $h_{2}(S) \rightarrow t_{2}(S)$ factors through a morphism $\rho: h_{2}(S) / h_{2}(C) \rightarrow t_{2}(S)$ and

Lemma 8.5. Assume $[C]^{2} \neq 0$ as above. Then

a) The diagram in $\mathcal{M}$

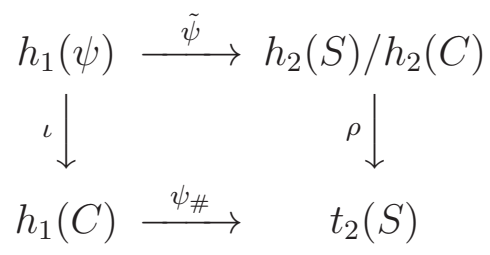


commutes. We write $\hat{\psi}:=\psi_{\#} \iota=\rho \tilde{\psi}$.

b) The morphisms $\tilde{\psi}$ and $\hat{\psi}$ do not depend on the choices of the (refined) $C K$ decompositions of $h(C)$ and $h(S)$.

c) In $\mathcal{M}_{\mathrm{alg}}, \hat{\psi}=0 \Longleftrightarrow \tilde{\psi}=0$.

Proof. a) We can use Diagram (8.7) to compute $\psi_{\#}$ and $\tilde{\psi}$ by using the section of $p_{C}$ and the retraction of $i_{S}$ given by $\pi_{1}^{C}$ and $\sum_{i>1} \pi_{i}^{S}$.

b) follows from a) and Lemma 3.1.

c) If $\rho \tilde{\psi}=0$ in $\mathcal{M}_{\text {alg, }}$, then $\tilde{\psi}$ factors in $\mathcal{M}_{\text {alg }}$ through $h_{2}^{\text {alg }}(S) / h_{2}(C)$. But $\mathcal{M}_{\text {alg }}\left(h_{1}(\psi), h_{2}^{\text {alg }}(S) / h_{2}(C)\right)=0$ by $(2.4)$ and Poincaré duality.

Given its importance in the sequel, we give a name to the motive $h_{1}(\psi) \otimes t_{2}(S)$ :

Notation 8.6. $M(\psi)=h_{1}(\psi) \otimes t_{2}(S)$.

Thus $M(\psi)$ is a direct summand of $h_{1}(C) \otimes t_{2}(S)$, and we have a similar decomposition to (8.3)

$$
M(\psi) \simeq t_{3}(\psi) \oplus h_{1}\left(B_{\psi}\right)(1)
$$

where $t_{3}(\psi)\left(\right.$ resp. $\left.B_{\psi}\right)$ is a direct summand of $t_{3}^{\prime}(C \times S)$ (resp. of the abelian variety $B$ of Corollary 8.3 a), up to isogeny). The proof is the same as for Lemma 5.1.

Definition 8.7. We call $B_{\psi}$ the exceptional summand of $B$ (or of $\left.J^{2}(C \times S)\right)$.

Remark 8.8. If $\psi_{1}$ is an epimorphism (which happens when $C$ is a smooth hyperplane section of $S$ ), the complementary summand of $B_{\psi}$, $B^{\prime}$, is such that $h_{1}\left(B^{\prime}\right)(1)$ is a direct summand of $h_{1}(S) \otimes t_{2}(S)$, and the largest of this type modulo numerical equivalence; so $B^{\prime}$ is independent of $\psi$ and (in principle) computable purely in terms of $S$. This justifies the terminology "exceptional".

8.3. Variation over a base. Let $X \in \mathbf{S m}(k)$. Recall DeningerMurre's category $\mathcal{M}(X)$ of relative Chow motives, constructed on smooth projective $X$-schemes [33]: it is 2-contravariant for morphisms in $\operatorname{Sm}(k)$. We shall need the following generalisation of the previous picture "over $X$ ":

- $S$ is a $k$-surface, to which we associate the constant relative $X$-surface $S_{X}:=S \times X \rightarrow X$;

- $C \rightarrow X$ is a relative curve, and $\psi \in C H^{2}\left(C \times_{X} S_{X}\right)=C H^{2}\left(C \times_{k}\right.$ $S)$ is a relative Chow correspondence. 
Then $C$ has a CK decomposition, defined as usual, and any refined CK decomposition of $h(S)$ pulls back to a refined CK decomposition of $S_{X}$. The modified class $[\psi]$ of (8.6) then makes sense in $\mathcal{M}(X)$, as well as the composition (8.8), whose bijectivity can be checked at any point of $X$. Moreover,

Lemma 8.9. Suppose $X$ connected, with generic point $\eta=\operatorname{Spec} K \stackrel{j}{\rightarrow}$ $X$. Then

a) The functor $j^{*}: \mathcal{M}(X) \rightarrow \mathcal{M}(K)$ is full.

b) If $C, C^{\prime}$ are two relative curves over $X$, the map

$$
\mathcal{M}(X)\left(h_{1}(C), h_{1}\left(C^{\prime}\right)\right) \rightarrow \mathcal{M}(K)\left(h_{1}\left(C_{\eta}\right), h_{1}\left(C_{\eta}^{\prime}\right)\right)
$$

is bijective, and the thick subcategory of $\mathcal{M}(X)$ generated by the $h_{1}(C)$ 's is abelian semi-simple.

Proof. a) follows immediately from the surjectivity of the map $C H^{*}(Y)$ $\rightarrow C H^{*}\left(Y_{\eta}\right)$ for any $Y \in \mathbf{S m}^{\text {proj }}(X)$. If $Z \subset X$ is a closed subset purely of codimension $c$, we have a more precise exact sequence

$$
C H^{i-c}\left(Y_{\mid Z}\right) \rightarrow C H^{i}(Y) \rightarrow C H^{i}\left(Y_{\mid X-Z}\right) \rightarrow 0 .
$$

For $c>1$, this implies an isomorphism $\mathcal{M}(X)\left(h(C), h\left(C^{\prime}\right)\right) \stackrel{\sim}{\rightarrow}$ $\mathcal{M}(X-Z)\left(h\left(C_{\mid X-Z}\right), h\left(C_{\mid X-Z}^{\prime}\right)\right)$, hence a fortiori the same isomorphism when replacing $h$ by $h_{1}$. For $c=1$, this isomorphism for the $h_{1}$ 's follows by a diagram chase from the formula

$\mathcal{M}(X)\left(h_{1}(C), h_{1}\left(C^{\prime}\right)\right) \simeq \operatorname{Coker}\left(C H^{1}(C) \oplus C H^{1}\left(C^{\prime}\right) \rightarrow C H^{1}\left(C \times_{X} C^{\prime}\right)\right)$

and the same one over $X-Z$. The first claim of b) follows from these two cases by passing to the limit, and the second claim follows from this and (2.5).

By Lemma $8.9 \mathrm{~b})$, the motive $h_{1}(\psi)$ of (8.9) makes sense in $\mathcal{M}(X)$ (note that we may write $h_{1}(S)$ as a direct summand of $h_{1}(D)$ for some smooth hyperplane section $D$ of $S$ ), as well as $\hat{\psi}$ and $M(\psi)$ (Lemma 8.5 and Notation 8.6). So does (8.10) when (8.8) is bijective; then Lemma 8.5 a) holds in $\mathcal{M}(X)$.

8.4. The coniveau filtration. Let $H$ be a Weil cohomology. For any $X \in \mathbf{S m}^{\text {proj }}$ and any $i \geq 0$, we have the coniveau filtration on $H^{i}(X)$ $[61,(2.0 .6)]$ :

$$
N^{j} H^{i}(X)=\sum_{(T, z)} \operatorname{Im}\left(H^{i-2 j}(T)(-j) \stackrel{\mathrm{cl}(z)^{*}}{\longrightarrow} H^{i}(X)\right)
$$


where $T \in \mathbf{S m}^{\text {proj }}$ and $z \in A_{H}^{\operatorname{dim} T+j}(X \times T)$. This generalises to any $M \in \mathcal{M}$ by the formula

$$
N^{j} H^{i}(M)=\sum_{(N, z)} \operatorname{Im}\left(H^{i-2 j}(N)(-j) \stackrel{\operatorname{cl}(z)^{*}}{\longrightarrow} H^{i}(M)\right)
$$

where $N \in \mathcal{M}$ and $z \in \mathcal{M}(M, N(j))$ : indeed, for $M=h(X)$, the images of (8.12) and (8.13) coincide as one sees by writing $N$ in (8.13) as a direct summand of some $h(T)$. (This formula makes it clear that $N^{*} H(M)$ is a descending filtration on $H(M)$.) Replacing the indexing sets $\mathcal{M}(M, N(j))$ by $\mathcal{M}(M, N(j)) \otimes F$, where $F$ is the field of coefficients of $H$, does not change the coniveau filtration. One may also use cycles modulo homological equivalence.

We note:

Lemma 8.10. Write $H^{3}(\psi):=H^{3}(M(\psi))$. Suppose that $N^{1} H^{3}(\psi)=$ 0. Then $B_{\psi}=0$, where $B_{\psi}$ is as in Definition 8.7 (see (8.11)).

Proof. By definition of the coniveau filtration, we have $H\left(h_{1}\left(B_{\psi}\right)(1)\right) \subseteq$ $N^{1} H^{3}(\psi)$. Therefore, the hypothesis implies that $h_{1}\left(B_{\psi}\right)(1)$ vanishes in $\mathcal{M}_{H}$. Hence so does $h_{1}\left(B_{\psi}\right)$, which implies that $B_{\psi}=0$ by $(2.5)$. 


\section{Part 2. Proofs of Theorems 2 and 3}

\section{THE $l$-ADIC ABEL-JACOBI MAP}

9.1. The $l$-adic realisation. Let $S$ be a scheme, and let $l$ be a prime number invertible on $S$. We write $\mathbf{S m}_{c}(S)$ for the category of smooth separated compactifiable morphisms $X \rightarrow S$ with morphisms proper $S$-morphisms, and $S_{c}\left(S, \mathbf{Q}_{l}\right)$ for the category of constructible $\mathbf{Q}_{l}$-adic sheaves over $S[49,1.4 .2], D_{c}^{b}\left(S, \mathbf{Q}_{l}\right)$ for the "derived category of constructible $\mathbf{Q}_{l}$-adic sheaves" ([30, 1.1.3], [37]) and

$$
R_{l, c}: \mathbf{S m}_{c}(S) \rightarrow D_{c}^{b}\left(S, \mathbf{Q}_{l}\right)
$$

for the (contravariant) functor which associates to $X \stackrel{f}{\rightarrow} S \in \mathbf{S m}_{c}(S)$ the object $R f_{!} \mathbf{Q}_{l}$. If $V$ is an $S$-scheme, we write

$$
\tilde{D}_{c}^{b}\left(V / S, \mathbf{Q}_{l}\right)=2-\underset{\mathcal{V}}{\lim } D_{c}^{b}\left(\mathcal{V}, \mathbf{Q}_{l}\right)
$$

where $V \rightarrow \mathcal{V}$ runs through the $S$-morphisms with $\mathcal{V}$ an $S$-scheme of finite type (see [43, Def. 6.3] for 2- $\underline{\lim }$ ). The functor $R_{l, c}$ factors through a functor

$$
\tilde{R}_{l, c}: \mathbf{S m}_{c}(V) \rightarrow \tilde{D}_{c}^{b}\left(V / S, \mathbf{Q}_{l}\right) .
$$

If $S=\operatorname{Spec} \mathbf{Z}[1 / l]$, we drop it from the notation.

With the definition of [37], the category $\tilde{D}_{c}^{b}\left(V / S, \mathbf{Q}_{l}\right)$ is triangulated and has a canonical $t$-structure with heart $\tilde{S}_{c}\left(V / S, \mathbf{Q}_{l}\right)=2-\underline{\lim }_{\mathcal{V}} S_{c}\left(\mathcal{V}, \mathbf{Q}_{l}\right)$. For $X \in \mathbf{S m}_{c}(V)$, we write $\tilde{H}_{l, c}^{i}(X / S)=H^{i}\left(\tilde{R}_{l, c}(X)\right)$; note that $\tilde{H}_{l, c}^{i}(X / S)$ $=\tilde{H}_{l}^{i}(X / S)$ when $X$ is proper (ordinary l-adic cohomology): in this case, this $S$-sheaf is locally constant by the smooth and proper base change theorem. Note the forgetful functor

$$
\omega_{V}^{l}: \tilde{S}_{c}\left(V, \mathbf{Q}_{l}\right) \rightarrow S_{c}\left(V, \mathbf{Q}_{l}\right)
$$

which forgets the "arithmetic monodromy action" to only remember the "geometric" one.

Suppose that $V=\operatorname{Spec} k$. The functor $\left(\tilde{R}_{l, c}\right)_{\mid \mathbf{S m}^{\mathrm{proj}}(k)}$ extends to an additive (contravariant) functor

$$
\tilde{R}_{l}: \mathcal{M}(k) \rightarrow \tilde{D}_{c}^{b}\left(k / S, \mathbf{Q}_{l}\right)
$$

such that

$$
\tilde{R}_{l}\left(h_{i}(X)\right)=\tilde{H}_{l}^{i}(X / S)[-i]
$$

for $X \in \mathbf{S m}^{\text {proj }}(k)$ and $i \in \mathbf{Z}$, for any CK decomposition of $X$. In particular, $\tilde{R}_{l}(\mathbb{L})=\mathbf{Q}_{l}(-1)[-2]$. 
Proposition 9.1. Let $M \in \mathcal{M}$. Suppose given a splitting $\tilde{R}_{l}(M) \simeq$ $\bigoplus_{i} \tilde{H}_{l}^{i}(M)[-i]$. Then, for any $n \geq 2$, the composition

$$
\begin{aligned}
& \mathcal{M}\left(M, \mathbb{L}^{n}\right) \stackrel{\tilde{R}_{l}}{\rightarrow} \operatorname{Hom}\left(\tilde{R}_{l}\left(\mathbb{L}^{n}\right), \tilde{R}_{l}(M)\right) \\
& \rightarrow \operatorname{Hom}\left(\tilde{R}_{l}\left(\mathbb{L}^{n}\right), \tilde{H}_{l}^{2 n-1}(M)[-2 n+1]\right) \simeq \operatorname{Ext}_{\tilde{S}_{c}\left(k / S, \mathbf{Q}_{l}\right)}^{1}\left(\mathbf{Q}_{l}(-n), \tilde{H}_{l}^{2 n-1}(M)\right) \\
& \quad \rightarrow \operatorname{Ext}_{\tilde{S}_{c}\left(k / S, \mathbf{Q}_{l}\right)}^{1}\left(\mathbf{Q}_{l}(-n), \tilde{H}_{l}^{2 n-1}(M) / N^{n-1} \tilde{H}_{l}^{2 n-1}(M)\right)
\end{aligned}
$$

factors through $\mathcal{M}_{\mathrm{alg}}\left(M, \mathbb{L}^{n}\right)$.

Proof. We may assume $k$ algebraically closed. Let $\left.\alpha \in \mathcal{M}\left(M, \mathbb{L}^{n}\right)\right)$ be such that $\alpha \mapsto 0 \in \mathcal{M}_{\text {alg }}\left(M, \mathbb{L}^{n}\right)$. Then there is a curve $D / k$ and classes $\beta \in J(D)(k)=\mathcal{M}\left(h_{1}(D), \mathbb{L}\right), \gamma \in \mathcal{M}\left(M, h_{1}(D)(n-1)\right)$ such that $\alpha$ factors as

$$
M \stackrel{\gamma}{\rightarrow} h_{1}(D)(n-1) \stackrel{\beta}{\rightarrow} \mathbb{L}^{n} .
$$

By definition of the coniveau filtration, the composition

$$
H_{l}^{1}(D)(n-1) \rightarrow H_{l}^{2 n-1}(M) \rightarrow H_{l}^{3}(M) / N^{n-1} H_{l}^{2 n-1}(M)
$$

is 0 , hence the conclusion.

As a special case, we get an Abel-Jacobi homomorphism

$$
\operatorname{Griff}^{2}(X) \otimes \mathbf{Q}_{l} \rightarrow \operatorname{Ext}_{\tilde{S}_{c}\left(k / S, \mathbf{Q}_{l}\right)}^{1}\left(\mathbf{Q}_{l}(-1), \tilde{H}_{l}^{3}(X) / N^{1} \tilde{H}_{l}^{3}(X)\right)
$$

for any smooth projective $X$. One can check that it is canonical: this was developed more integrally in the preliminary version of [53] (see [52, App. B]).

Remark 9.2. For $k=\mathbf{C}$, the same statement as Proposition 9.1 is valid when replacing the $l$-adic realisation by the Hodge realisation with values in the derived category of mixed Hodge structures, with the same proof.

9.2. A cohomological condition of nonvanishing. We come back to the situation of $\S 8.2$, and assume that $[C]^{2} \neq 0$.

Proposition 9.3. Take the notation of Lemma 8.5 a), Notation 8.6 and Lemma 8.10. Suppose that $N^{1} H_{l}^{3}(\psi)=0$. Then:

$$
\begin{aligned}
\tilde{R}_{l}(\hat{\psi}) \neq 0 \Rightarrow \hat{\psi} & \neq 0 \text { in } \mathcal{M}_{\text {alg }}\left(h_{1}(\psi), t_{2}(S)\right) \\
& \Rightarrow \psi_{\#} \neq 0 \text { in } \mathcal{M}_{\text {alg }}\left(h_{1}(C), t_{2}(S)\right) \simeq \operatorname{Griff}(C \times S) .
\end{aligned}
$$

Recall that the hypothesis also implies that $B_{\psi}=0$ (Lemma 8.10). 
Proof. The second implication is trivial. For the first, we use the rigidity of the category $\mathcal{M}\left[\mathbb{L}^{-1}\right]$ and the fact that $\tilde{R}_{l}$ is a symmetric monoidal functor. This yields a commutative diagram

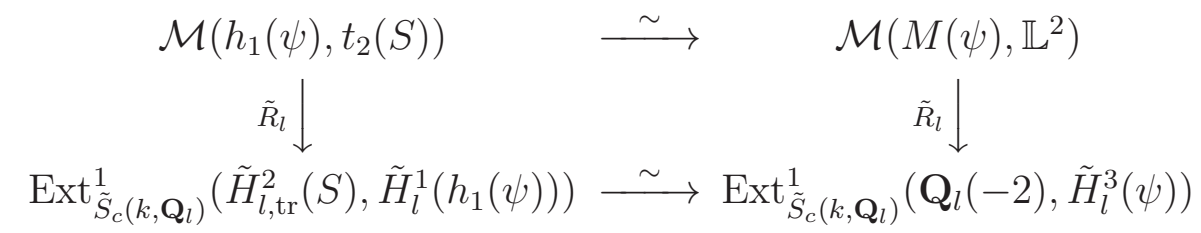

and we get the conclusion by taking $n=2$ in Proposition 9.1.

9.3. Interpretation of $\tilde{\psi}$ as an extension. We remain in the situation of $\S 8.2$, but assume further that $\psi$ is an embedding $C \hookrightarrow S$. For simplicity, we identify $C$ to its image in $S$. Let $V=S-C$; the exact triangle for cohomology with proper supports

$$
\tilde{R}_{l, c}(V) \rightarrow \tilde{R}_{l, c}(S) \stackrel{\psi^{*}}{\rightarrow} \tilde{R}_{l, c}(C) \stackrel{+1}{\longrightarrow}
$$

yields the corresponding long exact sequence

$$
\tilde{H}_{l}^{1}(S) \stackrel{\psi^{*}}{\rightarrow} \tilde{H}_{l}^{1}(C) \rightarrow \tilde{H}_{l, c}^{2}(V) \rightarrow \tilde{H}_{l}^{2}(S) \stackrel{\psi^{*}}{\rightarrow} \tilde{H}_{l}^{2}(C)
$$

whence a short exact sequence

$$
0 \rightarrow \tilde{H}_{l}^{1}(\psi) \rightarrow \tilde{H}_{l, c}^{2}(V) \rightarrow \tilde{H}_{l, \operatorname{prim}}^{2}(S) \rightarrow 0
$$

where $\tilde{H}_{l}^{1}(\psi)=\tilde{H}_{l}^{*}\left(h_{1}(\psi)\right)$ and $\tilde{H}_{l, \operatorname{prim}}^{2}(S)=\tilde{H}_{l}^{*}\left(h_{2}(S) / h_{2}(C)\right)$. This yields an extension class

$$
\mathcal{E} \in \operatorname{Ext}_{\tilde{S}_{c}\left(k, \mathbf{Q}_{l}\right)}^{1}\left(\tilde{H}_{l, \operatorname{prim}}^{2}(S), \tilde{H}_{l}^{1}(\psi)\right) .
$$

On the other hand, the morphism $\tilde{\psi}$ of (8.10) gives a morphism

$$
\left.\tilde{R}_{l, c}(\tilde{\psi}): \tilde{H}_{l, \text { prim }}^{2}(S)[-2] \rightarrow \tilde{H}_{l}^{1}(\psi)\right)[-1]
$$

which yields another extension class $\mathcal{E}^{\prime} \in \operatorname{Ext}_{\tilde{S}_{c}\left(k, \mathbf{Q}_{l}\right)}^{1}\left(\tilde{H}_{l, \text { prim }}^{2}(S), \tilde{H}_{l}^{1}(\psi)\right)$. The following proposition will not be used in the sequel, but is good to know:

Proposition 9.4. The classes $\mathcal{E}$ and $\mathcal{E}^{\prime}$ coincide.

Proof. This follows from applying the functor $\tilde{R}_{l, c}$ to Diagram (8.7). 


\section{Variations of Hodge structures}

10.1. The Hodge realisation. Let $X$ be a smooth connected complex algebraic curve. For any subring $A$ of $\mathbf{R}$, we have the category $\mathcal{V}(X, A)$ of good variations of mixed $A$-Hodge structures over $X([4, \S 4]$, see p. 8 for "good"). We shall write

$$
\mathcal{V}(X)=\operatorname{Im}(\mathcal{V}(X, \mathbf{Z}) \rightarrow \mathcal{V}(X, \mathbf{Q}))
$$

(variations of mixed Hodge structure of integral origin). This is a full tannakian subcategory of the (neutral) tannakian category $\mathcal{V}(X, \mathbf{Q})$ [84, Appendix].

Let $\operatorname{Loc}(X)$ be the tannakian category of local systems of finitedimensional $\mathrm{Q}$-vector spaces over $X$ (for the analytic topology). Let also $x \in X(\mathbf{C})$ be a rational point. We have a naturally commutative diagram of exact faithful $\otimes$-functors

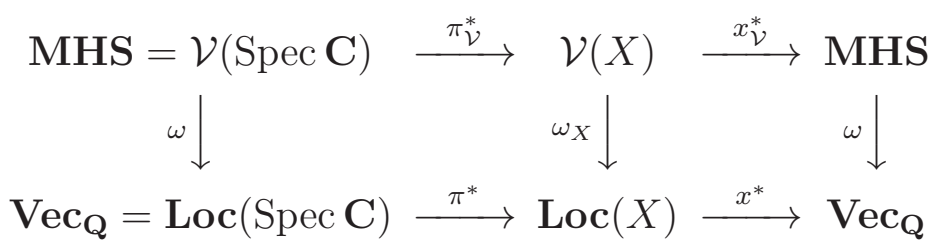

where $\pi: X \rightarrow$ Spec $\mathbf{C}$ is the structural morphism, MHS is the category of graded-polarisable mixed Q-Hodge structures, $\omega, \omega_{X}$ forget the Hodge structures and $x^{*} \pi^{*}=\operatorname{Id}_{\mathbf{V e c}_{\mathbf{Q}}}, x_{\mathcal{V}}^{*} \pi_{\mathcal{V}}^{*}=\operatorname{Id}_{\mathbf{M H S}}$.

The fibre functors $x^{*}$ and $\omega \circ x_{\mathcal{V}}^{*}$ define tannakian groups $\Pi_{1}(X, x)$ and $T_{x}$, which fit in a commutative diagram of affine Q-groups

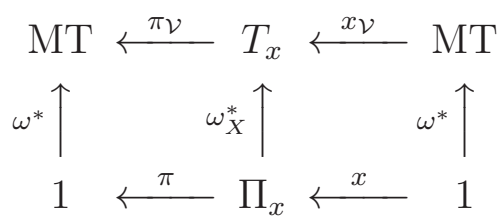

where MT is the Tannakian group of MHS (the universal MumfordTate group). In particular, $\pi_{\mathcal{V}}$ is split surjective. Note that $\Pi_{x}$ is the algebraic envelope of the topological fundamental group $\pi_{1}(X, x)$ (for its action on objects of $\operatorname{Loc}(X))$.

Theorem 10.1. $\omega_{X}^{*}\left(\Pi_{x}\right)$ is normal in $T_{x}$, and we have an exact sequence of affine $\mathbf{Q}$-groups

$$
\Pi_{x} \stackrel{\omega_{X}^{*}}{\longrightarrow} T_{x} \stackrel{\pi_{\mathcal{V}}}{\longrightarrow} \mathrm{MT} \rightarrow 1
$$

where $\pi_{\mathcal{V}}$ has the section $x_{\mathcal{V}}$.

For the proof, see Appendix C (Theorem C.11). Actually, we will use this theorem only in the case of pure variations of geometric origin, 
so that the difficult theorem of the fixed part of Schmid-SteenbrinkZucker used in the appendix (Theorem C.10) can be replaced with the much simpler theorem of the fixed part of Deligne [27, 4.1.1]. (I am indebted to Yves André for this clarification.)

Let $y \in X(\mathbf{C})$ be another point; any path from $x$ to $y$ yields an isomorphism of fibre functors $x^{*} \stackrel{\sim}{\rightarrow} y^{*}$; hence the collection of $\Pi_{x}$ and $T_{x}$ assemble to $X$-groupoids ${ }^{3} \Pi, T$, and (10.4) gets promoted to an exact sequence of $X$-groupoids

$$
\Pi \stackrel{\omega_{X}^{*}}{\longrightarrow} T \stackrel{\pi_{\mathcal{V}}}{\longrightarrow} \mathrm{MT} \rightarrow 1
$$

where MT is viewed as a constant $X$-groupoid. The sections $x_{\mathcal{V}_{*}} d o$ not define a splitting of $\pi_{\mathcal{V}}$ as a morphism of $X$-groupoids. The sequel of this subsection explains that this is "almost true" on integral objects of $\mathcal{V}(X)$.

For $V \in \mathcal{V}(X)$, write $T_{x}(V)$ for the image of $T_{x}$ in $\mathbf{G L}\left(\omega\left(V_{x}\right)\right)$, and $\Pi_{x}(V), \mathrm{MT}_{x}(V)$ for the images of $\omega_{X}^{*}\left(\Pi_{x}\right)$ and $x_{\mathcal{V}}(\mathrm{MT})$ : with the notation of $\left[4\right.$, Lemma 4], we have $\operatorname{MT}_{x}(V)=G_{x}$ and $\Pi_{x}(V)^{0}=H_{x}$ where ${ }^{0}$ means neutral component. We shall also need

Definition 10.2. An object $V \in \mathcal{V}(X)$ is connected if $\Pi_{x}(V)$ is connected for some (hence all) $x \in X(\mathbf{C})$.

Corollary 10.3. a) The collections $\left(T_{x}(V)\right)_{x \in X}$ and $\left(\Pi_{x}(V)\right)_{x \in X}$ define local systems on $X$. For any $x \in X(\mathbf{C}), \Pi_{x}(V)$ is normal in $T_{x}(V)$ and $\Pi_{x}(V)^{0}$ is normal in $T_{x}(V)^{0}$; we have

$$
\begin{aligned}
\Pi_{x}(V) \mathrm{MT}_{x}(V) & =T_{x}(V) \\
\Pi_{x}(V)^{0} \mathrm{MT}_{x}(V) & =T_{x}(V)^{0} \\
\pi_{0}\left(\Pi_{x}(V)\right) & \rightarrow \pi_{0}\left(T_{x}(V)\right) \text { is surjective. }
\end{aligned}
$$

b) For $x \in X(\mathbf{C})$, the following are equivalent:

(i) $\Pi_{x}(V)^{0} \subseteq \mathrm{MT}_{x}(V)$.

(ii) $\operatorname{MT}_{x}(V)=T_{x}(V)^{0}$.

If $V$ is simple and connected (Definition 10.2), then $V_{x}$ is simple for such $x$.

c) The set

$$
\operatorname{Exc}(V)=\{x \in X(\mathbf{C}) \mid \text { (i) and (ii) do not hold }\}
$$

is countable and its complement is path-connected; in particular, $x \mapsto$ $\mathrm{MT}_{x}(V)$ defines a local system on $X(\mathbf{C}) \backslash \operatorname{Exc}(V)$.

\footnotetext{
$3=$ functors from the fundamental groupoid $\pi_{1}(X)$ to the category of affine group schemes.
} 
Proof. In a), the first statements and (10.6) follow from Theorem 10.1 and $(10.5)$; (10.7) then follows from the connectedness of $\operatorname{MT}\left(V_{x}\right)$; finally, (10.8) means that $\Pi_{x}(V) T_{x}(V)^{0}=T_{x}(V)$, which follows from (10.6) and (10.7). This makes the equivalence in b) obvious, and the last assertion then follows from (10.8) and (ii). Finally, the proof of the second assertion of $[4$, Lemma 4] shows that $\operatorname{Exc}(V)$ is a union of countably many proper closed analytic subvarieties of $X(\mathbf{C})$, hence is countable since $\operatorname{dim}_{\mathbf{C}} X(\mathbf{C})=1$. For the path-connectedness, see $[74$, Lemma p. 276].

Remarks 10.4. a) In particular, $\Pi_{x}(V)^{0}$ is normal in $\operatorname{MT}_{x}(V)$ under the assumptions of $\mathrm{b}$ ). This is proven in [4, Th. 1], which also shows that $\Pi_{x}(V)^{0} \subseteq D\left(\mathrm{MT}_{x}(V)\right)$ (the derived subgroup). I learnt (10.8), the implication (i) $\Rightarrow$ (ii) and their consequence on the simplicity of $V_{x}$ from Y. André, whose proofs were different.

b) In fact, (10.8) is bijective: see Corollary C.14. We will not need this refinement.

10.2. Hodge coniveau. Let $V \in \mathcal{V}(X)$. We say that $V$ is effective if, for any $i$, the bundle $\left(\operatorname{gr}_{i}^{W} V\right)^{p, q}$ is $\neq 0$ only for $p \geq 0$ and $q \geq 0$. We write $\nu(V)=\sup \{n \in \mathbf{Z} \mid V(n)$ is effective $\}$. Whence the Hodge coniveau filtration:

$$
N_{H}^{j} V=\sum_{\substack{W \subseteq V \\ \nu(W) \geq j}} W \subseteq V .
$$

Let $\mathcal{V}(X)^{\text {eff }}$ be the full subcategory $\{V \in \mathcal{V}(X) \mid V$ is effective $\}$. Then $\nu(V) \geq j$ if and only if $V(j) \in \mathcal{V}(X)^{\text {eff }}$. Since $\mathcal{V}(X)^{\text {eff }}$ is closed under quotients, $N_{H}^{j}$ is right adjoint to the inclusion functor $\mathcal{V}(X)^{\mathrm{eff}}(j) \hookrightarrow \mathcal{V}(X)$, which implies the identity

$$
N_{H}^{j}\left(V_{1} \oplus V_{2}\right)=N_{H}^{j} V_{1} \oplus N_{H}^{j} V_{2} .
$$

One should beware that $N_{H}^{*}$ does not commute with specialisation: if $V \in \mathcal{V}(X)$, then the obvious inclusion, for $x \in X(\mathbf{C})$,

$$
\left(N_{H}^{j} V\right)_{x} \subseteq N_{H}^{j}\left(V_{x}\right)
$$

need not be an equality in general. However:

Proposition 10.5. For any $V \in \mathcal{V}(X)$, let

$$
\operatorname{Exc}^{N}(V)=\left\{x \in X \mid N_{H}^{1} V_{x} \neq 0\right\} .
$$

Suppose that $V$ is semi-simple, connected (Definition 10.2) and that $N_{H}^{1} V=0$. Then

$$
\operatorname{Exc}^{N}(V) \subseteq \bigcup_{S} \operatorname{Exc}(S)
$$


where Exc is as in Corollary 10.3 c) and $S$ runs through the simple constituents of $V$.

Proof. By (10.9) we have $\operatorname{Exc}^{N}\left(V_{1} \oplus V_{2}\right)=\operatorname{Exc}^{N}\left(V_{1}\right) \cup \operatorname{Exc}^{N}\left(V_{2}\right)$; since every direct summand of $V$ is connected, we may assume $V$ simple. Since $\operatorname{dim} V_{x}^{p, q}=\operatorname{rk} V^{p, q}$ for any $x$, we have $N_{H}^{1} V_{x} \neq V_{x}$ for any $x$. But $V_{x}$ is simple for $x \notin \operatorname{Exc}(V)$ by Corollary $\left.10.3 \mathrm{~b}\right)$, hence the conclusion.

The following easy lemma is a key step in the proof of Theorem 2: it is a special feature of Hodge theory.

Lemma 10.6. Let $V_{1}, V_{2} \in \mathcal{V}(X)$. Then $\nu\left(V_{1} \otimes V_{2}\right)=\nu\left(V_{1}\right)+\nu\left(V_{2}\right)$. If $V_{1}, V_{2} \in \mathcal{V}(X)^{\mathrm{eff}}$ and $V_{1} \otimes V_{2}$ is simple, then $\left(N_{H}^{1} V_{1}=0 \wedge N_{H}^{1} V_{2}=0\right)$ $\Rightarrow N_{H}^{1}\left(V_{1} \otimes V_{2}\right)=0$.

Proof. The inequality $\nu\left(V_{1} \otimes V_{2}\right) \geq \nu\left(V_{1}\right)+\nu\left(V_{2}\right)$ is obvious. By twisting, it suffices to show that $\nu\left(V_{1}\right)=0$ and $\nu\left(V_{2}\right)=0$ implies $\nu\left(V_{1} \otimes V_{2}\right)=0$. This is clear, since $\operatorname{gr}_{0}^{W}\left(V_{1}\right)^{0, q} \neq 0$ for some $q$ and $\operatorname{gr}_{0}^{W}\left(V_{2}\right)^{0, r} \neq 0$ for some $r$ implies $\operatorname{gr}_{0}^{W}\left(V_{1} \otimes V_{2}\right)^{0, q+r} \neq 0$. This readily implies the second claim.

For the next proposition, we write PHS $\subset$ MHS for the full subcategory of pure (polarisable) Hodge structures: it is semi-simple.

Proposition 10.7. Let $V \in \mathcal{V}(X)$. Assume that $\omega_{X}(V)$ is absolutely irreducible, in the sense that its endomorphism ring is $\mathbf{Q}$. Let $W \in$ PHS. Then:

a) If $W$ is simple, $V \otimes \pi_{\mathcal{V}}^{*} W$ is simple.

b) Suppose $V$ and $W$ effective. If $N_{H}^{1} V=0$ and $N_{H}^{1} W=0$, then $N_{H}^{1}\left(V \otimes \pi^{*} W\right)=0$. Moreover, $N_{H}^{1}\left(V_{x} \otimes W\right) \neq V_{x} \otimes W$ for any $x \in X$.

Proof. a) (I am grateful to http://mathoverflow .net/questions/208731 for its help in this proof.) We use Theorem 10.1. Take $x \in X$. We may view $V$ as a representation of $T_{x}$ and $W$ as a representation of MT. The restriction of $V \otimes \pi_{\mathcal{V}}^{*} W$ to $\Pi_{x}$ is semi-simple as a direct sum of copies of $V$, and its subrepresentations $E$ are in $1-1$ correspondence with the sub-vector spaces of $W$ by $E \mapsto\left(V^{*} \otimes E\right)^{\Pi_{X}} \subseteq \operatorname{End}_{\Pi_{x}}(V) \otimes W=W$ (using the absolute irreducibility of $\left.V_{\mid H}\right)$. Moreover, $E$ is a $T_{x}$-submodule of $V \otimes W$ if and only if $\left(V^{*} \otimes E\right)^{\Pi_{x}}$ is an MT-submodule of $W$.

b) This follows from a) (by reducing to $W$ simple) and Lemma 10.6. 
Corollary 10.8. Consider the hypotheses of Proposition 10.7 b). Suppose moreover $V$ connected. Then

$$
\operatorname{Exc}^{N}\left(V \otimes \pi_{\mathcal{V}}^{*} W\right) \subseteq \bigcup_{i} \operatorname{Exc}\left(V \otimes \pi_{\mathcal{V}}^{*} W_{i}\right)
$$

where $W_{i}$ runs through the irreducible components of $W, \operatorname{Exc}^{N}$ is as in Proposition 10.5 and Exc is as in Corollary 10.3 c). In particular, $\operatorname{Exc}^{N}\left(V \otimes \pi_{\mathcal{V}}^{*} W\right)$ is countable.

Proof. This follows from Proposition 10.7, Lemma 10.6 and Proposition 10.5 .

10.3. Comparison isomorphisms and coniveau filtrations. Let us go back to the category $\mathcal{M}(X)$ of relative Chow motives considered in $\S 8.3$. The functors

$$
\mathbf{S m}^{\text {proj }}(X) \ni(\mathcal{X} \stackrel{f}{\rightarrow} X) \mapsto R^{i} f_{*} \mathbf{Q} \in \mathcal{V}(X)
$$

induce a graded $\otimes$-functor

$$
\mathbf{H}_{B}^{*}: \mathcal{M}(X) \rightarrow \mathcal{V}^{*}(X)
$$

There is a similar enriched realisation functor

$$
\mathbf{H}_{l}^{*}: \mathcal{M}(X) \rightarrow \tilde{S}_{c}^{*}\left(X, \mathbf{Q}_{l}\right)
$$

to the graded category of "arithmetic" l-adic sheaves $\tilde{S}_{c}^{*}\left(X, \mathbf{Q}_{l}\right)$ of $\S 9.1$. Composing with the forgetful functors $\omega_{X}$ of (10.2) and $\omega_{X}^{l}$ from (9.1), we get relative Weil cohomologies

$$
H_{B}^{*}: \mathcal{M}(X) \rightarrow \operatorname{Loc}^{*}(X), \quad H_{l}^{*}: \mathcal{M}(X) \rightarrow \operatorname{Loc}^{*}\left(X, \mathbf{Q}_{l}\right)
$$

where $\operatorname{Loc}\left(X, \mathbf{Q}_{l}\right)$ is the category of local systems of $\mathbf{Q}_{l^{-}}$vector spaces. The comparison isomorphisms $R^{*} f_{*} \mathbf{Q}_{l} \simeq\left(R^{*} f_{*} \mathbf{Q}\right) \otimes \mathbf{Q}_{l}$ of $[28, \mathrm{~V}$, (3.5.1)] yield an isomorphism of functors

$$
H_{l}^{*} \simeq H_{B}^{*} \otimes_{\mathbf{Q}} \mathbf{Q}_{l} .
$$

For $M \in \mathcal{M}(X)$, we may define the coniveau filtration $N^{*}$ on $H_{B}^{*}(M)$ and $H_{l}^{*}(M)$ by Formula (8.13). Clearly

$$
N^{r} H_{B}^{j}(M) \subseteq N_{H}^{r} H_{B}^{j}(M):=\omega_{X}\left(N_{H}^{r} \mathbf{H}_{B}^{j}(X)\right)
$$

(we call the right hand side the Hodge coniveau filtration.)

We have the trivial lemma:

Lemma 10.9. The isomorphism (10.10) respects the coniveau filtrations. 
Let now $K / k$ be any regular extension, whence a functor $\mathcal{M}(k) \ni$ $M \mapsto M_{K} \in \mathcal{M}(K)$. If $l$ is a prime number $\neq$ char $k$ and $M \in \mathcal{M}$, we have an isomorphism

$$
H_{l}(M) \stackrel{\sim}{\rightarrow} H_{l}\left(M_{K}\right)
$$

by invariance of $l$-adic cohomology under separably closed base change.

Proposition 10.10 (cf. [61, Prop. 2.2]). The isomorphism (10.12) respects the coniveau filtrations.

Proof. Let $X \in \mathbf{S m}^{\text {proj }}(k)$. By definition of the coniveau filtration, the isomorphism $H_{l}^{i}(X) \stackrel{\sim}{\rightarrow} H_{l}^{i}\left(X_{K}\right)$ yields an inclusion $N^{j} H_{l}^{i}(X) \subseteq$ $N^{j} H_{l}^{i}\left(X_{K}\right)$ for all $j \geq 0$. Let $x \in H_{l}^{i}(X)$ be such that $x_{K} \in N^{j} H_{l}^{i}\left(X_{K}\right)$. There is $Y \in \mathbf{S m}^{\operatorname{proj}}(K), y \in H_{l}^{i-2 j}(Y)(-j)$ and a correspondence $\alpha \in C H_{\operatorname{dim} X-j}\left(X_{K} \times_{K} Y\right)$ such that $x_{K}=\alpha^{*} y$. Spread $Y$ to a smooth projective $f: \mathcal{Y} \rightarrow S$ with $S \in \operatorname{Sm}(k)$, and lift $\alpha$ to $\tilde{\alpha} \in$ $C H_{\operatorname{dim} X-j+\operatorname{dim} S}\left(X_{S} \times{ }_{S} \mathcal{Y}\right)$. For a closed point $s \in S$, we have $y_{s}=$ $s p_{s}(y) \in H_{l}^{i-2 j}\left(\mathcal{Y}_{s}\right)$ and $\alpha_{s}=y^{*}(\tilde{\alpha}) \in C H_{\operatorname{dim} X-j}\left(X \times_{k} \mathcal{Y}_{s}\right)$, where $\mathcal{Y}_{s}=f^{-1}(s)$; obviously, $x_{k(y)}=\alpha_{s}^{*} y_{s}$.

We may now state

Proposition 10.11. Let $X$ be a smooth connected algebraic $\mathbf{C}$-curve and $M \in \mathcal{M}(X)$. Suppose that $N_{H}^{j} \mathbf{H}_{B}^{i}\left(M_{U}\right)=0$ for some $(i, j)$ and any nonempty Zariski open subset $U \subseteq X$, where $M_{U}$ is the base change of $M$ to $U^{4}$. Let $M_{\eta} \in \mathcal{M}(K)$ be the "generic fibre" of $M$, where $K=\mathbf{C}(X)$. Then we have $N^{j} H_{l}^{i}\left(M_{\eta}\right)=0$ for any prime number $l$.

Proof. We have to show that any morphism $\varphi: M_{\eta} \rightarrow N(j)$ in $\mathcal{M}(K)$ induces 0 on $l$-adic cohomology. We may find $U$ such that $\varphi$ comes from a morphism $\varphi_{U}: M_{U} \rightarrow \mathcal{N}(j)$, with $\mathcal{N} \in \mathcal{M}(U)$. It suffices to show that $\varphi_{U}$ induces 0 on $l$-adic cohomology, and by Lemma 10.9 we may replace the latter by Betti cohomology. This now follows from the inclusion (10.11) and the hypothesis.

\section{LEFsChetz PENCILS}

Our aim in this section is to prove the generic case of Theorem 2 (see Corollary 11.6).

\footnotetext{
${ }^{4}$ One can show that the case $U=X$ implies the general case.
} 
11.1. Preparations. Let $S \in \operatorname{Sm}^{\text {proj }}(k)$ be a geometrically connected surface. Consider a Lefschetz pencil [29, (5.6)]:

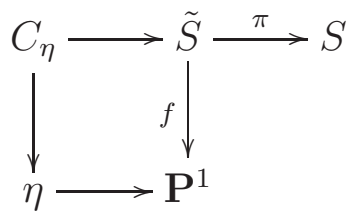

where $\tilde{S}$ is the incidence variety and $\eta$ is the generic point of $\mathbf{P}^{1}$.

We have the closed immersion

$$
\psi: \tilde{S} \hookrightarrow S \times \mathbf{P}^{1} .
$$

Let $U \subset \mathbf{P}^{1}$ be the smooth locus of $f$; the restriction $\psi_{U}: \tilde{S}_{U} \hookrightarrow$ $S \times U$ of $\psi$ to $U$ yields a relatively ample smooth divisor, and the formalism of $\S 8.3$ applies. Thus we get motives

$$
h_{1}\left(\psi_{U}\right), M\left(\psi_{U}\right)=h_{1}\left(\psi_{U}\right) \otimes t_{2}(S \times U / U) \in \mathcal{M}(U)
$$

and a morphism

$$
\widehat{\psi_{U}}: h_{1}\left(\psi_{U}\right) \rightarrow t_{2}(S \times U / U)
$$

(see Lemma 8.5).

Let $l$ be a prime number. In $H_{l}^{1}\left(\tilde{S}_{U}\right)$, we have two sub-local systems:

- $H_{l}^{1}\left(\psi_{U}\right):=H_{l}^{1}\left(h_{1}\left(\psi_{U}\right)\right)$;

- $\underline{E}$, the local system of vanishing cycles [29, (6.1)].

We assume that $\underline{E} \neq 0$ : it is possible up to composing the given projective embedding of $S$ with a Veronese embedding, cf. [60, Cor. 6.4]. This forces $U \neq \mathbf{P}^{1}$, hence $U$ is affine. Then:

Proposition 11.1. a) The two local systems $H_{l}^{1}\left(\psi_{U}\right)$ and $\underline{E}$ are canonically isomorphic.

b) For $u \in U$, the algebraic envelope of the monodromy action on $H_{l}^{1}\left(\psi_{U}\right)_{u}=H_{l}^{1}\left(\psi_{u}\right)$ is $\mathbf{S p}\left(H_{l}^{1}\left(\psi_{u}\right)\right)$, where $\mathbf{S p}$ is with respect to the symplectic pairing induced by the isomorphism of a).

c) If $k \subseteq \mathbf{C}$, the same picture holds when replacing $H_{l}$ by $H_{B}$. In particular, $H_{B}^{1}(\psi) \in \mathcal{V}\left(\mathbf{P}^{1}\right)$ (cf. (10.1)).

Proof. a) Write $f_{U}$ for the restriction of $f$ to $U$ and $g_{U}$ for the projection $S \times U \rightarrow U$. By definition, we have an exact sequence

$$
R^{1}\left(g_{U}\right)_{*} \mathbf{Q}_{l} \rightarrow R^{1}\left(f_{U}\right)_{*} \mathbf{Q}_{l} \rightarrow H_{l}^{1}\left(\psi_{U}\right) \rightarrow 0 .
$$

For $u \in U$, let $C_{u}=f^{-1}(u)$ and $E_{u} \subset H_{l}^{1}\left(C_{u}\right)$ be the subspace of vanishing cycles $[29,(5.2)]$, i.e. the fibre of $\underline{E}$ at $u$. The PicardLefschetz formula $[29,(5.8)$ a) 3$)]$ shows that $E_{u}^{\perp}=H_{l}^{1}\left(C_{u}\right)^{\pi_{1}^{\text {et }}(U, u)}$, and the Hard Lefschetz theorem (which, for a surface, follows algebraically 
from (6.2) for $i=1$ ) implies that $E_{u}$ is nondegenerate for the Poincaré duality pairing. Therefore, it suffices to show that $H_{l}^{1}\left(C_{u}\right)^{\pi_{1}^{\text {et }}(U, u)}=$ $\operatorname{Im}\left(H_{l}^{1}\left(S_{k(u)}\right) \rightarrow H_{l}^{1}\left(C_{u}\right)\right)$ for any $u \in U$; since these are local systems, it suffices to show this at one point $u$, that we choose to be the generic point $\eta$.

Since $U$ is affine, $U_{\bar{k}}$ has cohomological dimension 1 and the Leray spectral sequence for $f_{U}$ yields an epimorphism

$$
H_{l}^{1}(\tilde{S}) \longrightarrow H_{l}^{0}\left(U, R^{1}\left(f_{U}\right)_{*} \mathbf{Q}_{l}\right)=H_{l}^{1}\left(C_{\eta}\right)^{\pi_{1}^{\text {et }}(U, \eta)} .
$$

But $\pi: \tilde{S} \rightarrow S$ is a blow-up, hence $H_{l}^{1}(S) \stackrel{\sim}{\rightarrow} H_{l}^{1}(\tilde{S})$ and $H_{l}^{1}(S) \rightarrow$ $H_{l}^{1}\left(C_{\eta}\right)^{\pi_{1}^{\text {et }}(U, \eta)}$ is surjective; so is a fortiori $H_{l}^{1}\left(S_{k(\eta)}\right) \rightarrow H_{l}^{1}\left(C_{\eta}\right)^{\pi_{1}^{\text {et }}(U, \eta)}$.

b) now follows from a) and the Kazhdan-Margulis theorem [29, Th. (5.10)], and the first statement of c) follows from (10.10); the second one holds because the vanishing cycles are integrally defined.

More easily:

Lemma 11.2. If $k \subseteq \mathbf{C}$, we have $N_{H}^{1} H_{B, \operatorname{tr}}^{2}(S)=0$.

Proof. The Lefschetz $(1,1)$ theorem shows that $N^{1} H_{B}^{2}(S)=N_{H}^{1} H_{B}^{2}(S)$, and $N^{1} H_{B, \operatorname{tr}}^{2}(S)=N^{1} H_{B}^{2}(S) \cap H_{\mathrm{tr}}^{2}(S)=0$ by definition of $H_{\mathrm{tr}}^{2}(S)$.

\subsection{The main results.}

Theorem 11.3. Suppose char $k=0$. Then,

a) $N^{1} H_{l}^{3}\left(\psi_{U^{\prime}}\right)=0$ for any nonempty open subset $U^{\prime} \subseteq U$, and $N^{1} H_{l}^{3}\left(\psi_{u}\right)$ $\neq H_{l}^{3}\left(\psi_{u}\right)$ for all $u \in U_{(0)}$.

b) $N^{1} H_{l}^{3}\left(\psi_{\eta}\right)=0$.

c) If $k \subseteq \mathbf{C}$, there exists a countable subset $E(\psi) \subset U(\mathbf{C})$ such that, for any $u \in U(k) \backslash E(\psi)$, one has $N^{1} H_{l}^{3}\left(\psi_{u}\right)=0$.

d) For $u=\eta$ or $u \in U(k) \backslash E(\psi)$, one has $B_{\psi_{u}}=0$ where $B_{\psi_{u}}$ is the exceptional part of $J^{2}\left(C_{u} \times S_{k(u)}\right)$ (see Definition 8.7).

Proof. For a), using Lemma 10.9 and Proposition 10.10 we reduce to $k$ finitely generated over $\mathbf{Q}$, then to $k=\mathbf{C}$, and then replace $l$-adic cohomology by Betti cohomology. It suffices to prove the statements with $N^{1}$ replaced by $N_{H}^{1}$. We apply Proposition 10.7 b) by taking $V=H_{B}^{1}\left(\psi_{U^{\prime}}\right), W=H_{B, \mathrm{tr}}^{2}(S)$ : Proposition 11.1 (resp. Lemma 11.2) gives its hypothesis for $V$ (resp. $W$ ) - note that the vanishing of $N_{H}^{1} H_{B}^{1}\left(\psi_{U^{\prime}}\right)$ is trivial. b) then follows from Proposition 10.11. Since $V$ is connected by Proposition 11.1, c) follows from Corollary 10.8. Finally, d) follows from the above and Lemma 8.10. 
Remark 11.4. I don't know if Theorem $11.3 \mathrm{~b}$ ) holds in positive characteristic. By contrast, the next result holds over any $k$.

Theorem 11.5 (cf. [41, Th. 2]). Suppose that $b^{2}>\rho$. Then $\tilde{R}_{l}\left(\widehat{\psi_{\eta}}\right) \neq$ 0 .

The proof is given in the next subsection.

Corollary 11.6. Theorem 2 (i) is true.

Proof. This follows from Theorem 11.3 b), Theorem 11.5 and Proposition 9.3.

Remark 11.7. Applying (3.6) with $Y=\tilde{S}$ and using (3.3), we get

$$
\begin{aligned}
T\left(S_{k(\tilde{S})}\right) / T(S) \simeq \mathcal{M}\left(h_{1}(\tilde{S}), t_{2}(S)\right) \oplus \mathcal{M}\left(t_{2}(\tilde{S}), t_{2}(S)\right) \\
\simeq \mathcal{M}\left(h_{1}(S), t_{2}(S)\right) \oplus \operatorname{End}_{\mathcal{M}}\left(t_{2}(S)\right) .
\end{aligned}
$$

The first isomorphism comes again from (3.3) plus the isomorphisms (6.2); the second one comes from the fact that $h_{1}(\tilde{S}) \stackrel{\sim}{\rightarrow} h_{1}(S)$ and $t_{2}(\tilde{S}) \stackrel{\sim}{\rightarrow} t_{2}(S)$ since $\tilde{S} \rightarrow S$ is a birational morphism. On the other hand, $T(S) \stackrel{\sim}{\rightarrow} T\left(S_{k\left(\mathbf{P}^{1}\right)}\right)$, either directly or by $(3.7)$ since $h_{1}\left(\mathbf{P}^{1}\right)=0$. Letting $K=k\left(\mathbf{P}^{1}\right)$, we thus get

$$
\begin{aligned}
\mathcal{M}(K)\left(h_{1}\left(C_{\eta}\right), t_{2}\left(S_{K}\right)\right) & \simeq T\left(S_{k(\tilde{S})}\right) / T\left(S_{K}\right) \\
& \simeq \mathcal{M}\left(h_{1}(S), t_{2}(S)\right) \oplus \operatorname{End}_{\mathcal{M}}\left(t_{2}(S)\right)
\end{aligned}
$$

and, using (8.1), we find a surjection in $\mathbf{A b} \otimes \mathbf{Q}$ :

$$
\mathcal{M}\left(h_{1}(S), t_{2}(S)\right) \oplus \operatorname{End}_{\mathcal{M}}\left(t_{2}(S)\right) \longrightarrow \operatorname{Griff}\left(C_{\eta} \times_{K} S_{K}\right) .
$$

For simplicity, assume $b^{1}=0$ : this is the case e.g. if $S$ is a K3 surface. Then $h_{1}(S)=0$ and the strong form of Bloch's conjecture [16, Conj. 1.8]:

$$
\operatorname{End}_{\mathcal{M}}\left(t_{2}(S)\right) \stackrel{\sim}{\rightarrow} \operatorname{End}_{\mathcal{M}_{\text {hom }}}\left(t_{2}(S)\right) ?
$$

predicts that $\operatorname{dim}_{\mathbf{Q}} \operatorname{Griff}\left(C_{\eta} \times_{K} S_{K}\right) \otimes \mathbf{Q}<\infty$.

11.3. Proof of Theorem 11.5. Without loss of generality, we may and do assume $k$ algebraically closed.

Let $V \subseteq \mathbf{P}^{1}$ be an open subset, and let $\tilde{S}_{V}=f^{-1}(V)$. The projection $f_{V}: \tilde{S}_{V} \rightarrow V$ yields a trace map

$$
H_{l}^{2}\left(\tilde{S}_{V}\right) \stackrel{\left(f_{V}\right)_{*}}{\longrightarrow} H^{0}(V)(-1) .
$$

Lemma 11.8. Let $j_{V}$ be the open immersion $\tilde{S}_{V} \hookrightarrow \tilde{S}$, and let $a$ : $H_{l, \mathrm{tr}}^{2}(\tilde{S}) \hookrightarrow H_{l}^{2}(\tilde{S})$ be induced by $\pi_{2}^{S, \mathrm{tr}}$. Then: 
a) The composition $H_{l, \mathrm{tr}}^{2}(\tilde{S}) \stackrel{a}{\rightarrow} H_{l}^{2}(\tilde{S}) \stackrel{j_{V}^{*}}{\rightarrow} H_{l}^{2}\left(\tilde{S}_{V}\right)$ is injective.

b) The composition $\left(f_{V}\right)_{*} j_{V}^{*} a$ is 0 .

Proof. a) By semi-purity, Ker $j_{V}^{*}$ is contained in the image of $\operatorname{NS}(\tilde{S}) \otimes$ $\mathrm{Q}_{l}(-1) \stackrel{\text { cl }}{\rightarrow} H_{l}^{2}(\tilde{S})$. The claim follows since $\operatorname{Im} a$ is the orthogonal complement of this image, by construction of $\pi_{2}^{S, \operatorname{tr}}[56, \S 7.2]$.

b) It suffices to handle the case $V=\mathbf{P}^{1}$. Let $x \in H_{l, \mathrm{tr}}^{2}(\tilde{S})$ and $y \in H_{l}^{2}\left(\mathbf{P}^{1}\right)$. By the projection formula, we have

$$
\left\langle f_{*} a x, y\right\rangle_{\mathbf{P}^{1}}=\left\langle a x, f^{*} y\right\rangle_{\tilde{V}}=0
$$

for the same reason as in the proof of (1). Thus $f_{*} a x=0$.

In Lemma 11.8, suppose $V \subseteq U$. In particular $V$ is affine, hence has étale cohomological dimension 1 and the Leray spectral sequence for the projection $f_{V}: \tilde{S}_{V} \rightarrow V$ yields a short exact sequence

$$
\begin{aligned}
0 \rightarrow H^{1}\left(V, R^{1}\left(f_{V}\right)_{*} \mathbf{Q}_{l}\right) \rightarrow H_{l}^{2}\left(\tilde{S}_{V}\right) & \\
& \stackrel{\varepsilon_{V}}{\longrightarrow} H^{0}\left(V, R^{2}\left(f_{V}\right)_{*} \mathbf{Q}_{l}\right) \rightarrow 0
\end{aligned}
$$

where the edge homomorphism $\varepsilon_{V}$ coincides with the trace map of (11.6). By Lemma 11.8, and (11.7), the map $a$ then yields an injection

$$
\tilde{a}_{V}: H_{l, \mathrm{tr}}^{2}(\tilde{S}) \hookrightarrow H^{1}\left(V, R^{1}\left(f_{V}\right)_{*} \mathbf{Q}_{l}\right) .
$$

Let $h_{1}\left(\psi_{V}\right)$ denote the restriction of $h_{1}\left(\psi_{U}\right)$ to $\mathcal{M}(V)$ (see $(11.3)$ ), and let $H_{l}^{1}\left(\psi_{V}\right)=H_{l}\left(h_{1}\left(\psi_{V}\right)\right) \in S_{c}\left(V, \mathbf{Q}_{l}\right)$ be its realisation.

Lemma 11.9. Let $j_{V}$ be the inclusion $V \hookrightarrow \mathbf{P}^{1}$. The image of $\tilde{a}_{V}$ is contained in $H^{1}\left(V, j_{V}^{*} j_{*} \underline{E}\right) \simeq H^{1}\left(V, H_{l}^{1}\left(\psi_{V}\right)\right)$.

Proof. The Leray spectral sequence for $f$ :

$$
H^{p}\left(\mathbf{P}^{1}, R^{q} f_{*} \mathbf{Q}_{l}\right) \Rightarrow H^{p+q}\left(\tilde{S}, \mathbf{Q}_{l}\right)=: H^{p+q}(\tilde{S})
$$

yields a 3-step filtration $F^{p} H^{2}(\tilde{S})$ on $H^{2}(\tilde{S})$ with successive quotients $H^{0}\left(\mathbf{P}^{1}, R^{2} f_{*} \mathbf{Q}_{l}\right) \simeq \mathbf{Q}_{l}(-1), H^{1}\left(\mathbf{P}^{1}, R^{1} f_{*} \mathbf{Q}_{l}\right)$ and $H^{2}\left(\mathbf{P}^{1}, f_{*} \mathbf{Q}_{l}\right)=$ $H^{2}\left(\mathbf{P}^{1}, \mathbf{Q}_{l}\right)=\mathbf{Q}_{l}(-1)$. By Lemma $\left.11.8 \mathrm{~b}\right)$, we have $\operatorname{Im} a \subset F^{1} H^{2}(\tilde{S})$.

Let $j: U \hookrightarrow \mathbf{P}^{1}$ be the inclusion. Then $R^{1} f_{*} \mathbf{Q}_{l} \stackrel{\sim}{\rightarrow} j_{*} j^{*} R^{1} f_{*} \mathbf{Q}_{l}$ $[29,(5.8)]$ and, by the proof of Proposition 11.1 a), the orthogonal complement of $j_{*} \underline{E}$ in $R^{1} f_{*} \mathbf{Q}_{l}$ is constant, hence

$$
H^{1}\left(\mathbf{P}^{1}, j_{*} \underline{E}\right) \stackrel{\sim}{\rightarrow} H^{1}\left(\mathbf{P}^{1}, R^{1} f_{*} \mathbf{Q}_{l}\right) .
$$

Therefore, the image of $\tilde{a}_{V}$ is contained in $H^{1}\left(V, j_{V}^{*} j_{*} \underline{E}\right)$. But we have $j_{V}^{*} j_{*} \underline{E} \simeq H_{l}^{1}\left(\psi_{V}\right)$ by Proposition $\left.11.1 \mathrm{a}\right)$. 
Note that the closed immersion $\psi$ of (11.2) factors as

$$
\tilde{S} \stackrel{\gamma}{\rightarrow} \tilde{S} \times \mathbf{P}^{1} \stackrel{\Pi}{\rightarrow} S \times \mathbf{P}^{1}
$$

where $\gamma$ is the graph of $f$ and $\Pi=\pi \times 1_{\mathbf{P}^{1}}$. Restricting to the open subset $V \subseteq U$, we get an induced factorisation

$$
\tilde{S}_{V} \stackrel{\gamma_{V}}{\longrightarrow} \tilde{S} \times V \stackrel{\Pi_{V}}{\longrightarrow} S \times V
$$

and $\gamma_{V}$ induces a morphism $\widehat{\gamma_{V}}: h_{1}\left(\gamma_{V}\right) \rightarrow t_{2}(\tilde{S} \times V / V)=t_{2}(\tilde{S})_{V}$ analogous to (11.4), whence a morphism in $D_{c}^{b}\left(V, \mathrm{Q}_{l}\right)$

$$
H_{l, \mathrm{tr}}^{2}(\tilde{S})_{V}[-2] \rightarrow H_{l}^{1}\left(\gamma_{V}\right)[-1]
$$

with $H_{l}^{1}\left(\gamma_{V}\right)=H_{l}\left(h_{1}\left(\gamma_{V}\right)\right)$, as usual. Applying $\operatorname{Hom}\left(\mathbf{Q}_{l}[-2],-\right)$ to (11.8), we get a homomorphism

$$
H_{l, \mathrm{tr}}^{2}(\tilde{S})=H^{0}\left(V, H_{l, \mathrm{tr}}^{2}(\tilde{S})_{V}\right) \rightarrow H^{1}\left(V, H_{l}^{1}\left(\gamma_{V}\right)\right) .
$$

Write $\widehat{a_{V}}: H_{l, \mathrm{tr}}^{2}(\tilde{S}) \hookrightarrow H^{1}\left(V, H_{l}^{1}\left(\psi_{V}\right)\right)$ for the injection given by Lemma 11.9. Then

Lemma 11.10. a) The direct summands $h_{1}\left(\psi_{V}\right)$ and $h_{1}\left(\gamma_{V}\right)$ of $h_{1}\left(\tilde{S}_{V}\right)$ coincide.

b) In view of a), the homomorphism (11.9) is equal to $\widehat{a_{V}}$, up to sign.

Proof. a) By definition, $h_{1}\left(\psi_{V}\right)=\operatorname{Ker}\left(h_{1}\left(\tilde{S}_{V}\right) \stackrel{\left(\psi_{V}\right)_{*}}{\longrightarrow} h_{1}(S \times V / V)\right)$, and similarly for $h_{1}\left(\gamma_{V}\right)$. But $\left(\Pi_{V}\right)_{*}: h_{1}(\tilde{S} \times V / V) \rightarrow h_{1}(S \times V / V)$ is split since $\pi$ is a blow-up with smooth centre.

b) Under $R_{l}$, the CK decomposition of $h\left(\tilde{S}_{V} / V\right)$ yields a direct sum decomposition in $D_{c}^{b}\left(V, \mathbf{Q}_{l}\right)$

$$
R\left(f_{V}\right)_{*} \mathbf{Q}_{l} \simeq \bigoplus_{q=0}^{2} R^{q}\left(f_{V}\right)_{*} \mathbf{Q}_{l}[-q]
$$

whence a splitting of the Leray spectral sequence

$$
H^{n}\left(\tilde{S}_{V}, \mathbf{Q}_{l}\right)=D_{c}^{b}\left(V, \mathbf{Q}_{l}\right)\left(\mathbf{Q}_{l}, R\left(f_{V}\right)_{*} \mathbf{Q}_{l}\right) \simeq \bigoplus_{q=0}^{2} H^{n-q}\left(V, R^{q}\left(f_{V}\right)_{*} \mathbf{Q}_{l}\right)
$$

from which the claim follows easily.

End of proof of Theorem 11.5. We have a naturally commutative diagram of categories and functors

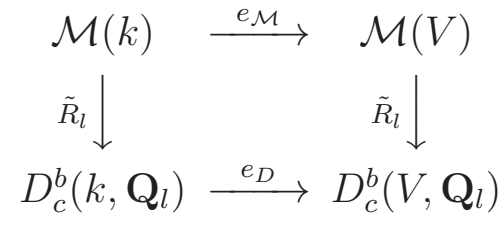


where $D_{c}^{b}\left(k, \mathbf{Q}_{l}\right)$ is simply the bounded derived category of $\mathbf{V e c}_{\mathbf{Q}_{l}}$. The composite homomorphism

$$
\begin{aligned}
\mathcal{M}(k)\left(t_{2}(S), t_{2}(S)\right) \stackrel{e_{\mathcal{M}}}{\longrightarrow} \mathcal{M}(V)\left(t_{2}(S)_{V}, t_{2}(S)_{V}\right) \\
\quad \stackrel{{\widehat{\psi_{V}}}^{*}}{\longrightarrow} \mathcal{M}(V)\left(h_{1}\left(\psi_{V}\right), t_{2}(S)_{V}\right)
\end{aligned}
$$

sends tautologically $1_{t_{2}(S)}$ to $\widehat{\psi_{V}}$. Therefore, the composite homomorphism

$$
\begin{aligned}
& \operatorname{End}_{\mathbf{Q}_{l}}\left(H_{l, \mathrm{tr}}^{2}(S)\right)=\operatorname{End}_{D_{c}^{b}\left(k, \mathbf{Q}_{l}\right)}\left(H_{l, \mathrm{tr}}^{2}(S)[-2]\right) \\
& \stackrel{e_{D}}{\longrightarrow} \operatorname{End}_{D_{c}^{b}\left(V, \mathbf{Q}_{l}\right)}\left(H_{l, \mathrm{tr}}^{2}(S)_{V}[-2]\right) \\
& \stackrel{\left.\widehat{R_{l}\left(\psi_{V}\right.}\right)_{*}}{\longrightarrow} D_{c}^{b}\left(V, \mathbf{Q}_{l}\right)\left(H_{l, \mathrm{tr}}^{2}(S)_{V}[-2], H_{l}^{1}\left(\psi_{V}\right)[-1]\right)
\end{aligned}
$$

sends $1_{H_{l, \mathrm{tr}}^{2}(S)}$ to $R_{l}\left(\widehat{\psi_{V}}\right)$. The hypothesis $b^{2}>\rho$ means that $1_{H_{l, \mathrm{tr}}^{2}(S)} \neq$ 0 , hence, to conclude, it suffices to show that (11.10) is injective for any $V \subseteq U$. Since $V$ is irreducible, $e_{D}$ is an isomorphism. To show the injectivity of $\widehat{R_{l}\left(\psi_{V}\right)_{*}}$, we may replace the left (constant) term $H_{l, \mathrm{tr}}^{2}(S)_{V}[-2]$ by $\mathrm{Q}_{l}[-2]$, and are left to show that the map

$$
\begin{aligned}
D_{c}^{b}\left(V, \mathbf{Q}_{l}\right)\left(\mathbf{Q}_{l}[-2], H_{l, \mathrm{tr}}^{2}(S)_{V}[-2]\right) & \\
& \stackrel{\widehat{R_{l}\left(\psi_{V}\right)_{*}}}{\longrightarrow} D_{c}^{b}\left(V, \mathbf{Q}_{l}\right)\left(\mathbf{Q}_{l}[-2], H_{l}^{1}\left(\psi_{V}\right)[-1]\right)
\end{aligned}
$$

is injective.

But $\pi: \tilde{S} \rightarrow S$ is a birational morphism, hence $t_{2}(\pi)$ is an isomorphism by (3.3) and [57, Cor. 2.4.2]; thus we are reduced to the injectivity of the map

$$
\begin{aligned}
H_{l, \mathrm{tr}}^{2}(\tilde{S})= & D_{c}^{b}\left(V, \mathbf{Q}_{l}\right)\left(\mathbf{Q}_{l}[-2], H_{l, \mathrm{tr}}^{2}(\tilde{S})_{V}[-2]\right) \\
& \stackrel{\left.\widehat{R_{l}\left(\gamma_{V}\right.}\right)_{*}}{\longrightarrow} D_{c}^{b}\left(V, \mathbf{Q}_{l}\right)\left(\mathbf{Q}_{l}[-2], H_{l}^{1}\left(\gamma_{V}\right)[-1]\right)=H^{1}\left(V, H_{l}^{1}\left(\gamma_{V}\right)\right)
\end{aligned}
$$

where we used Lemma 11.10 a) to identify $H_{l}^{1}\left(\psi_{V}\right)$ with $H_{l}^{1}\left(\gamma_{V}\right)$. This map is none else than (11.9). The conclusion now follows from Lemma $11.10 \mathrm{~b})$.

Remark 11.11. This fills in details in [41, Proof of Th. 2]. (The reader should beware that there are typos in this proof.) The main add-on is Lemma 11.10 b).

\section{Specialisation}

In this section, $k$ is a subfield of $\mathbf{C}$. 
12.1. Coniveau and Lefschetz pencils. We keep the notation of Section 11. We shall use the following application of a theorem due independently to Serre [83, 10.6, theorem] and Terasoma [89, Th. 2]:

Proposition 12.1 (cf. $[41, \S 2]$ ). Let $k$ be a field of characteristic $\neq l$, and let

$$
0 \rightarrow V \rightarrow E \rightarrow W \rightarrow 0
$$

be a nonsplit extension of l-adic representations of $G_{k(t)}$ (the absolute Galois group of $k(t))$. We assume that $E$ is unramified away from a finite set $S$ of places of $k(t) / k$. Then the set

$$
\Omega=\left\{x \in \mathbf{A}^{1}(k) \backslash S \mid E_{x} \text { is split }\right\}
$$

is thin. (See [83, 9.1, Def.] for the definition of thin.)

Proof. Let $G=\operatorname{Im}\left(G_{k(t)} \rightarrow G L(E)\right)$ : this is an $l$-adic Lie group. By either of the two references quoted before the proposition, the set $\Omega^{\prime}$ of $x \in \mathbf{A}^{1}(k) \backslash S$ such that the decomposition group $G_{x}$ at $x$ is not equal to $G$ is thin.

Remark 12.2. By a transfer argument, the condition $\left(G: G_{x}\right)<\infty$ is sufficient for $E_{x}$ not to be split.

Corollary 12.3. The set

$$
\left\{u \in U(k) \mid R_{l}\left(\widehat{\psi_{u}}\right)=0\right\}
$$

is thin.

Proof. This follows from Theorem 11.5 and Proposition 12.1, applied to the extension

$$
0 \rightarrow H_{l}^{1}\left(\psi_{\eta}\right) \rightarrow E \rightarrow H_{l, \mathrm{tr}}^{2}(S) \rightarrow 0
$$

defined by $R_{l}\left(\widehat{\psi_{\eta}}\right)$.

Lemma 12.4. Let $K_{1}$ be a field of characteristic 0 . Then $K=K_{1}(t)$ is Hilbertian (see [83, 9.5, Def.] for the definition of Hilbertian). Moreover, $\operatorname{card}\left(\mathbf{A}^{1}(K)-\Omega\right)=\operatorname{card}(K)$ for any thin subset $\Omega \subset \mathbf{A}^{1}(K)$.

Proof. The first statement is [83, 9.5, Rk. 5]; the second one follows from examining its proof. Namely, $\{a t+b \mid a, b \in K\} \backslash \Omega \supseteq U(K)$ for a suitable nonempty open subset $U \subseteq \mathbf{A}_{K}^{1} \times \mathbf{A}_{K}^{1}$.

Theorem 12.5. Suppose $k=\mathrm{C}$. Let

$$
\operatorname{Exc}^{\mathcal{M}}(\psi)=\left\{u \in U(\mathbf{C}) \mid\left(\psi_{u}\right)_{\#}=0 \in \operatorname{Griff}\left(C_{u} \times S\right) \otimes \mathbf{Q}\right\} .
$$

Then $U(\mathbf{C}) \backslash \operatorname{Exc}^{\mathcal{M}}(\psi)$ is uncountable. 
Proof. There is an uncountable subfield $K \subset \mathbf{C}$, of the form $K_{1}(t)$, such that our Lefschetz pencil is defined over $K$. Indeed, the Lefschetz pencil is defined over some finitely generated subfield $K_{0} \subset \mathbf{C}$; pick a transcendence basis $\left(t_{\alpha}\right)_{\alpha \in A}$ of $\mathbf{C} / K_{0}$, and let $K_{1}=K_{0}\left(\left(t_{\alpha}\right)_{\alpha \in A-\{\beta\}}\right)$ where $\beta \in A$ is chosen, and $K=K_{0}\left(t_{\alpha}\right)$. The claim now follows from Theorem 11.3 c), Corollary 12.3, Lemma 12.4 and Proposition 9.3.

12.2. André's motives. Proposition 12.1 is sufficient to get Corollary 12.3 over a finitely generated field $k$, but not to ensure that the set $U(k) \backslash E(\psi)$ of Theorem $11.3 \mathrm{c})$ is nonempty. For this, we now have to pass from Mumford-Tate groups as in Theorem 10.3 to motivic Galois groups in the sense of André [6]. Moreover, we have to strengthen the hypothesis on $S$.

We write $\operatorname{Mot}(k)$ for the category denoted by $\mathcal{M}_{k}$ in loc. cit., 4.5, Ex. (i). This is a semi-simple Q-linear Tannakian category [6, Th. $0.4]$, provided with canonical $\otimes$-functors

$$
\mathcal{M}(k) \rightarrow \operatorname{Mot}(k) \stackrel{\text { H}}{\rightarrow} \mathbf{P H S} \stackrel{\omega}{\rightarrow} \operatorname{Vec}_{\mathbf{Q}}
$$

refining the Hodge realisation on $\mathcal{M}(k)$, where $\mathcal{M}(k)$ denotes as before the category of effective Chow motives; to agree with the present convention and the one in [6], the first functor is contravariant and the second one is covariant. For $X \in \operatorname{Sm}^{\operatorname{proj}}(k)$, we write $h_{\text {mot }}(X)$ for the image in $\operatorname{Mot}(k)$ of $h(X) \in \mathcal{M}(k)$, in order to avoid confusion.

The fibre functor $\omega$ (resp. $\omega \circ \mathbf{H}$ ) defines a Tannakian group $\mathrm{MT}_{\text {red }}$ (resp. Gal): the absolute Mumford-Tate group (resp. the absolute motivic Galois group [6, 4.6]); the first one is the largest proreductive quotient of the group MT considered (for all mixed Hodge structures) in $\S 10.1$. The functor $\mathbf{H}$ induces a homomorphism of proreductive groups

$$
\mathrm{MT}_{\text {red }} \rightarrow \mathrm{Gal} .
$$

Let $M \in \operatorname{Mot}(k)$. Write $\operatorname{Gal}(M)$ (resp. $\mathrm{MT}(M)$ ) for the image of Gal (resp. of $\mathrm{MT}_{\text {red }}$ ) in $\mathbf{G L}(H(M)$ ), where $H=\omega \mathbf{H}$. The above homomorphism induces a corresponding monomorphism of reductive groups (not necessarily connected on the right hand side)

$$
\operatorname{MT}(M) \hookrightarrow \operatorname{Gal}(M) .
$$

Warning 12.6. The first functor of (12.1) is monoidal but not symmetric monoidal, since the commutativity constraint has been modified in $\operatorname{Mot}(k)$ [6, 4.3] but not in $\mathcal{M}(k)$. We shall only use its structure as a functor, not as a $\otimes$-functor.

We need a further definition: 
Definition $12.7([6,6.1])$. An object $M \in \operatorname{Mot}(k)$ is of abelian type if $M$ belongs to the Tannakian subcategory $\mathbf{M o t}_{\mathrm{ab}}(k)$ generated by motives of abelian varieties and Artin motives. A smooth projective variety $X$ is of motivated abelian type if $h_{\text {mot }}(X)$ is of abelian type.

Examples 12.8. a) Any abelian variety, or of any product of curves, is of motivated abelian type. Similarly for Fermat varieties [59]. A more recent example is the Fano surface of lines of a cubic 3-fold [34]. (All this is already true in $\mathcal{M}(k)$.)

b) A K3 surface (hence an Enriques surface) is of motivated abelian type [6, Th. 7.1]. So is a smooth cubic hypersurface of dimension $\leq 6$ (loc. cit., Th. 7.2), and a surface of general type verifying $p_{g}=K^{2}=1$ [7, Cor. 1.5.2].

c) Let $f: X \rightarrow Y$ be a surjective morphism. If $X$ is of motivated abelian type, so is $Y\left(h_{\text {mot }}(Y)\right.$ is a direct summand of $h_{\text {mot }}(X)$.)

d) To be of motivated abelian type is a birationally invariant property for smooth projective varieties of dimension $\leq 3$.

e) A motive $M \in \operatorname{Mot}(k)$ is in $\operatorname{Mot}_{\mathrm{ab}}(k)$ if and only if it is a direct summand of $N \otimes h_{\text {mot }}(A)(r)$ for some abelian variety $A$, some Artin motive $N$ and some $r \geq 0$. (Hint: if $A$ and $B$ are abelian varieties, $h_{\text {mot }}(A)$ and $h_{\text {mot }}(B)$ are direct summands of $h_{\text {mot }}(A \times B)$; the Artin motive $\mathbb{L}$ is a direct summand of $h(E)$ for any elliptic curve $E$.)

The main properties of $\operatorname{Mot}_{\mathrm{ab}}(k)$ that we shall use are the following:

Theorem 12.9. Suppose $k$ algebraically closed.

a) In (12.1), the restriction of $\mathbf{H}$ to $\mathbf{M o t}_{\mathrm{ab}}(k)$ is fully faithful. In particular, for $M \in \mathbf{M o t}_{\mathrm{ab}}(k)$, any direct summand of $\mathbf{H}(M)$ is of the form $\mathbf{H}(N)$, where $N$ is a direct summand of $M$.

b) For $M \in \operatorname{Mot}_{\mathrm{ab}}(k)$, the homomorphism (12.2) is an isomorphism.

Proof. By rigidity, a) is a translation of [6, Th. 0.6.2] (any Hodge cycle on an abelian variety is motivated), and b) follows from a).

12.3. Hodge variations and motivated variations. Let $X$ be a smooth connected $k$-curve. It is convenient to use the category $\operatorname{Mot}(X)$ of relative André motives introduced by Arapura and Dhillon in [9] (and denoted by $M_{A}(X)^{\text {str }}$ in loc. cit.): its construction relies on André's deformation theorem for motivated cycles [6, Th. 0.5]. A family of (motivated) motives in the sense of $[6, \S \S$ before Th. 5.2] defines an object of $\operatorname{Mot}(X)$. This category is still Q-linear, Tannakian, semisimple and provided with a faithful, exact $\otimes$-functor

$$
\operatorname{Mot}(X) \stackrel{\text { H}}{\rightarrow} \mathcal{V}_{\mathbf{Z}}\left(X_{\mathbf{C}}\right) \text {. }
$$


Indeed, this is part of [9, Th. 2.3], except for the fact that $\mathbf{H}(M)$ is good and of integral origin for any $M \in \operatorname{Mot}(X)$. The first fact follows from the polarizability of $\mathbf{H}(M)$ (cf. [4, (1) p. 9]). For the second fact, write $M$ as a direct summand of $h_{\text {mot }}(\mathcal{X})(n)$, where $f: \mathcal{X} \rightarrow X$ is smooth projective and $n \geq 0$. If $p$ is the corresponding projector, then $\mathbf{H}(M)$ is a direct summand via $\mathbf{H}(p)$ of $\bigoplus_{i \geq 0} R^{i} f_{*} \mathbf{Q}(n)=$ $\left(\bigoplus_{i \geq 0} R^{i} f_{*} \mathbf{Z}(n)\right) \otimes \mathbf{Q}$. If $d$ is a positive integer such that $d p$ has integral coefficients, then $\mathbf{H}(M)=\operatorname{Im}(\mathbf{H}(d p)) \otimes \mathbf{Q}$.

The functor $\mathcal{M}(k) \rightarrow \operatorname{Mot}(k)$ of (12.1) similarly generalises to a functor

$$
\mathcal{M}(X) \rightarrow \operatorname{Mot}(X)
$$

where $\mathcal{M}(X)$ is as in $\S 8.3$.

Suppose $k=\mathbf{C}$; let $M \in \mathbf{M o t}(X)$ and $V=\mathbf{H}(M)$. With notation as before Corollary 10.3, we write $T_{x}(M), \mathrm{MT}_{x}(M), \Pi_{x}(M)$ for $T_{x}(V), \mathrm{MT}_{x}(V), \Pi_{x}(V)$. To this notation, we add $\operatorname{Gal}_{x}(M):=\operatorname{Gal}\left(M_{x}\right)$ and define

\section{Definition 12.10.}

$$
\begin{aligned}
\operatorname{Exc}_{\mathbf{H}}(M) & =\operatorname{Exc}(\mathbf{H}(M))(\text { see Cor. } 10.3 \mathrm{c})) ; \\
\operatorname{Exc}_{\text {mot }}(M) & =\left\{x \in X(\mathbf{C}) \mid \Pi_{x}(M)^{0} \nsubseteq \mathrm{Gal}_{x}(M)\right\} \\
\operatorname{Exc}_{\mathbf{H}}^{N}(M) & =\operatorname{Exc}^{N}(\mathbf{H}(M))(\text { see Prop. 10.5) } \\
\operatorname{Exc}_{\operatorname{mot}}^{N}(M) & =\left\{x \in X(\mathbf{C}) \mid N^{1} H\left(M_{x}\right) \neq 0\right\} .
\end{aligned}
$$

By (12.2), we have

$$
\operatorname{Exc}_{\text {mot }}(M) \subseteq \operatorname{Exc}_{\mathbf{H}}(M)
$$

Similarly

$$
\operatorname{Exc}_{\text {mot }}^{N}(M) \subseteq \operatorname{Exc}_{\mathbf{H}}^{N}(M)
$$

since $N^{1} H(M) \subseteq N_{\mathbf{H}}^{1} H(M)$. Finally, by Proposition 10.5:

$$
\operatorname{Exc}_{\mathbf{H}}^{N}(M) \subseteq \bigcup_{S} \operatorname{Exc}(S)
$$

where $S$ runs through the simple summands of $\mathbf{H}(M)$, provided $\mathbf{H}(M)$ is connected (see Definition 10.2 for connected).

Definition 12.11. An object $M \in \operatorname{Mot}(X)$ is of abelian type if $M_{x}$ is of abelian type for every $x \notin \operatorname{Exc}_{\text {mot }}(M) .{ }^{5}$ We write $\operatorname{Mot}_{\mathrm{ab}}(X)$ for the corresponding full subcategory of $\operatorname{Mot}(X)$.

\footnotetext{
${ }^{5} \mathrm{I}$ don't know if one $x$ is sufficient.
} 
Theorem 12.12. Let $M \in \operatorname{Mot}_{\mathrm{ab}}(X)$ and $N \in \operatorname{Mot}_{\mathrm{ab}}(\mathbf{C})$. Suppose $\mathbf{H}(M)$ absolutely simple and connected. Then

$$
\operatorname{Exc}_{\text {mot }}^{N}\left(M \otimes \pi^{*} N\right) \subseteq \bigcup_{S} \operatorname{Exc}_{\text {mot }}\left(M \otimes \pi^{*} S\right)
$$

where $S$ runs through the simple summands of $N$.

Proof. Clearly, $\mathbf{H}\left(M \otimes \pi^{*} N\right)$ is connected (it has the same monodromy as $\mathbf{H}(M))$. In view of (12.5), (12.6) and Proposition 10.7 a), it therefore suffices to show that

$$
\bigcup_{S} \operatorname{Exc}_{\operatorname{mot}}\left(M \otimes \pi^{*} S\right)=\bigcup_{\Sigma} \operatorname{Exc}\left(\mathbf{H}(M) \otimes \pi^{*} \Sigma\right)
$$

where $\Sigma$ runs through the simple summands of $\mathbf{H}(N)$. By Theorem 12.9 a), any $\Sigma$ is of the form $\mathbf{H}(S)$ for some $S$. For such an $S$, let $x \notin$ $\operatorname{Exc}_{\text {mot }}\left(M \otimes \pi^{*} S\right)$. By Theorem $\left.12.9 \mathrm{~b}\right), \operatorname{MT}_{x}\left(M \otimes \pi^{*} S\right)=\operatorname{Gal}_{x}(M \otimes$ $\left.\pi^{*} S\right)$, hence $x \notin \operatorname{Exc}_{\mathbf{H}}\left(M \otimes \pi^{*} S\right)$ and we are done by (12.4).

12.4. $l$-adic variations. We come back to the case where $k$ is an arbitrary subfield of $\mathbf{C}$. Write $\bar{k}$ for its algebraic closure in C. For $M \in \operatorname{Mot}(X)$, we define

$$
\begin{aligned}
& \operatorname{Exc}_{\text {mot }}(M)=\operatorname{Exc}_{\text {mot }}\left(M_{\mathbf{C}}\right) \cap X(\bar{k}) \\
& \operatorname{Exc}_{\text {mot }}^{N}(M)=\operatorname{Exc}_{\text {mot }}^{N}\left(M_{\mathbf{C}}\right) \cap X(\bar{k})
\end{aligned}
$$

cf. Definition 12.10. For $d \geq 1$, we also write

$$
X(\bar{k})^{\leq d}=\{x \in X(\bar{k}) \mid[k(x): k] \leq d\}
$$

and

$$
\begin{aligned}
& \operatorname{Exc}_{\text {mot }}(M)^{\leq d}=\operatorname{Exc}_{\text {mot }}(M) \cap X(\bar{k})^{\leq d} \\
& \operatorname{Exc}_{\text {mot }}^{N}(M)^{\leq d}=\operatorname{Exc}_{\text {mot }}^{N}(M) \cap X(\bar{k})^{\leq d} .
\end{aligned}
$$

By [9, Th. 2.3], there is another realisation functor

$$
R_{l}^{\operatorname{mot}}: \operatorname{Mot}(X) \rightarrow \tilde{S}_{c}\left(X, \mathbf{Q}_{l}\right)
$$

where $\tilde{S}_{c}\left(X, \mathbf{Q}_{l}\right)$ is as in $\S 9.1$. The tannakian theory of this category is parallel to that of $\mathcal{V}\left(X_{\mathbf{C}}\right)$ as developed in $\S 10.1$ : the rôle of $\operatorname{Loc}(X)$ is played by $S_{c}\left(X, \mathrm{Q}_{l}\right)$ (see (9.1)); for $\bar{x}$ a geometric point of $X$, the proalgebraic $\mathbf{Q}$-groups $\Pi_{x}, T_{x}$ and $\mathrm{MT}$ are replaced by the pro-algebraic $\mathbf{Q}_{l^{-}}$ groups $\Pi_{\bar{x}}^{l}, T_{\bar{x}}^{l}$ and $\pi^{l}$ which are the Zariski envelopes (for $l$-adic representations) respectively of the geometric fundamental group $\pi_{1}^{\text {et }}\left(X_{\bar{k}}, \bar{x}\right)$, the absolute fundamental group of $\pi_{1}^{\text {et }}(X, \bar{x})$ and $G_{k}:=\operatorname{Gal}(\bar{k} / k)$, with 
a similar exact sequence to (10.4). ${ }^{6}$ If we write similarly $\pi^{l}(k(x), \bar{x})$ for the Zariski envelope of $\pi_{1}^{\text {et }}(\operatorname{Spec} k(x), \bar{x})$, the homomorphism $x_{\mathcal{V}}$ is replaced by a homomorphism $\bar{x}_{S}: \pi^{l}(k(x), \bar{x}) \rightarrow T_{\bar{x}}^{l}$, where $x$ is the point of $X$ underlying $\bar{x}$.

To be more specific, here is an $l$-adic analogue to Corollary 10.3, where $V \in S_{c}\left(X, \mathbf{Q}_{l}\right)$ :

Proposition 12.13. a) The collections $\left(T_{x}^{l}(V)\right)_{x \in X}$ and $\left(\Pi_{x}^{l}(V)\right)_{x \in X}$ define local systems on $X_{\text {ét. }}$ Writing $\pi_{x}^{l}(V)$ for the image of $\bar{x}_{S}$ in $G L\left(V_{x}\right)$, we have

$$
\begin{aligned}
& \Pi_{x}(V) \pi_{x}^{l}(V) \text { is open in } T_{x}(V), \text { with equality if } x \in X(k) \\
& \Pi_{x}(V)^{0} \pi_{x}^{l}(V)^{0}=T_{x}(V)^{0}
\end{aligned}
$$

b) For $x \in X_{(0)}$, the following are equivalent:

(i) $\Pi_{x}^{l}(V)^{0} \subseteq \pi_{x}^{l}(V)$.

(ii) $\pi_{x}^{l}(V)$ is open in $T_{x}^{l}(V)$.

The functor (12.9) defines a homomorphism

$$
\pi^{l} \rightarrow \mathrm{Gal} \otimes_{\mathbf{Q}} \mathbf{Q}_{l}
$$

inducing a monomorphism for $M \in$ Mot and $x \in X_{(0)}$ :

$$
\pi_{x}^{l}(M) \hookrightarrow \operatorname{Gal}_{x}(M) \otimes_{\mathbf{Q}} \mathbf{Q}_{l}
$$

analogous to (12.2). Since $\pi_{1}^{\text {ét }}\left(X_{\bar{k}}\right)$ is canonically isomorphic to the profinite completion of $\pi_{1}(X(\mathbf{C}))$, this yields an inclusion, for $M \in$ $\operatorname{Mot}(X)$ :

$$
\operatorname{Exc}_{\text {mot }}(M) \subseteq \operatorname{Exc}_{l}\left(R_{l}(M)\right)
$$

where, for $V \in S_{c}\left(X, \mathbf{Q}_{l}\right)$

$$
\begin{aligned}
\operatorname{Exc}_{l}(V) & =\left\{x \in X(\bar{k}) \mid \Pi_{x}^{l}(V)^{0} \not \pi_{x}^{l}(V)\right\} \\
& =\left\{x \in X(\bar{k}) \mid \pi_{x}^{l}(V) \text { is not open in } T_{x}^{l}(V)\right\}
\end{aligned}
$$

cf. Proposition 12.13 b). This yields:

Theorem 12.14. Suppose that $k$ is finitely generated over $\mathbf{Q}$.

a) [19, Th. 5.1] Let $M \in \operatorname{Mot}(X)$. Then $\operatorname{Exc}_{\text {mot }}(M)^{\leq d}$ is finite for any $d \geq 1$.

b) Let $M \in \operatorname{Mot}_{\mathrm{ab}}(X)$ and $N \in \operatorname{Mot}(k)$; assume that $N_{\bar{k}} \in \operatorname{Mot}_{\mathrm{ab}}(\bar{k})$. Then the set $\operatorname{Exc}_{\text {mot }}^{N}\left(M \otimes \pi^{*} N\right) \leq d$ is finite for any $d \geq 1$.

\footnotetext{
${ }^{6}$ We write in this subsection $\pi_{1}^{\text {ét }}$ for the étale fundamental group, in order to avoid confusion with the topological fundamental group $\pi_{1}$ of $\S 10.1$.
} 
Proof. By [21, Th. 5.8] and [22, Th. 1.1], the sets $\operatorname{Exc}_{l}\left(R_{l}(M)\right) \leq d$ are finite (see next subsection for more details), hence a) follows from (12.13). Theorem 12.12 then implies b).

12.5. GLP representations. In this subsection, we recall results of Cadoret-Tamagawa which were just used in the proof of Theorem 12.14, and prove two lemmas which will be used in the next subsection.

Definition 12.15 ([21]). Let $X$ be a scheme separated, geometrically connected and of finite type over $k$. An $l$-adic representation $\rho: \pi_{1}^{\text {et }}(X) \rightarrow G L_{m}\left(\mathbf{Q}_{l}\right)$ of the étale fundamental group of $X$ is geometrically Lie perfect (GLP) if the Lie algebra of the $l$-adic Lie group $\rho\left(\pi_{1}\left(X_{\bar{k}}\right)\right)$ is perfect (i.e., its abelianisation is 0 ).

With the notation of (12.14), we have by [22, Th. 1.1]:

Theorem 12.16. The set $\operatorname{Exc}_{l}(\rho) \leq d$ is finite for all $d \geq 1$ if $\rho$ is GLP and $k$ is finitely generated over $\mathbf{Q}$.

By [21, Th. 5.8], the monodromy action of $\pi_{1}^{\text {ét }}(X)$ on $R^{*} f_{*} \mathbf{Q}_{l}$ is GLP if $\operatorname{dim} X=1$ and $f: Y \rightarrow X$ is a smooth proper morphism. This explains the proof of Theorem 12.14 a) in a more detailed way.

We shall need:

Lemma 12.17 (see also [20, Lemma 2.4]). Let

$$
0 \rightarrow \underline{E} \rightarrow V \rightarrow \pi^{*} W \rightarrow 0
$$

be an extension in $S_{c}\left(X, \mathbf{Q}_{l}\right)$, where $W \in S_{c}\left(k, \mathbf{Q}_{l}\right)$. Assume that $\underline{E}$ is GLP and semi-simple, and that the fixed points of $\underline{E}$ under any open subgroup of the monodromy group are trivial. Then $V$ is GLP.

Proof. Fix $x \in X$. Let $G_{\underline{E}}\left(\right.$ resp. $\left.G_{V}\right)$ be the monodromy group of $\underline{E}$ (resp. $V$ ) at $x$. We have an exact sequence

$$
1 \rightarrow N \rightarrow G_{V} \stackrel{p}{\rightarrow} G_{\underline{E}} \rightarrow 1
$$

where $N$ embeds into $\operatorname{Hom}\left(\pi^{*} W, \underline{E}\right)$. In particular, $N$ is abelian, $G_{\underline{E}}$ acts on $N$ and the properties of the representation $\underline{E}$ carry over to $N$. Abelianising the short exact sequence of Lie algebras

$$
0 \rightarrow N=\operatorname{Lie}(N) \rightarrow \operatorname{Lie}\left(G_{V}\right) \rightarrow \operatorname{Lie}\left(G_{\underline{E}}\right) \rightarrow 0
$$

we therefore get another short exact sequence

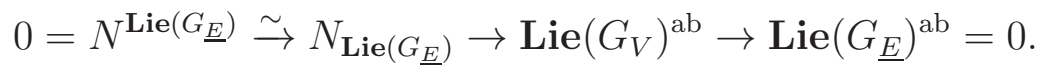

Hence $\operatorname{Lie}\left(G_{V}\right)^{\text {ab }}=0$, as desired. 
Lemma 12.18. Let $\rho: \pi_{1}^{\text {ét }}(X) \rightarrow G L_{m}\left(\mathbf{Q}_{l}\right)$ be any l-adic representation, and let $K$ be an extension of $k$. Then $\operatorname{Exc}_{l}(\rho)=\operatorname{Exc}_{l}\left(\rho_{K}\right)$. Here $\rho_{K}: \pi_{1}^{\text {et }}\left(X_{K}\right) \rightarrow G L_{m}\left(\mathbf{Q}_{l}\right)$ is the representation deduced from $\rho$ via the projection $\pi_{1}^{\text {et }}\left(X_{K}\right) \rightarrow \pi_{1}^{\text {ét }}(X)$.

Proof. We may assume $K / k$ finitely generated. Write $K=k(U)$, where $U$ is smooth, and let $\bar{K}$ be an algebraic closure of $K$. Then a point $x \in X(\bar{K})$ spreads to a $k$-morphism $\varphi: V \rightarrow X$, where $V$ is étale over $U$. If $x \notin X(\bar{k}), \varphi$ is dominant (since $\operatorname{dim} X=1$ ), hence $\operatorname{Im}\left(\varphi_{*}\right.$ : $\left.\pi_{1}^{\text {ét }}(V) \rightarrow \pi_{1}^{\text {ét }}(X)\right)$ is open and $x \notin \operatorname{Exc}_{l}(\rho)$.

12.6. Finiteness. We go back to the situation of Subsection 12.4. We assume $S_{\bar{k}}$ to be of motivated abelian type.

Proposition 12.19. Suppose $k$ is finitely generated over Q. Write $M_{\text {mot }}\left(\psi_{U}\right)$ for the image of $M\left(\psi_{U}\right) \in \mathcal{M}(U)$ in $\operatorname{Mot}(U)$ by the functor of $(12.3)$ (see (11.3) for $\left.M\left(\psi_{U}\right)\right)$. Then the set $\operatorname{Exc}_{\text {mot }}^{N}\left(M_{\text {mot }}\left(\psi_{U}\right)\right) \leq d$ (12.8) is finite for any $d \geq 1$.

Proof. This is the special case $M=h_{\text {mot }}^{1}\left(\psi_{U}\right), N=t_{\text {mot }}^{2}(S \times U / U)$ of Theorem 12.14 b).

Proposition 12.20. a) The local system $R_{l}\left(\widehat{\psi_{U}}\right) \in \tilde{S}_{c}\left(U, \mathbf{Q}_{l}\right)$ is GLP. b) For any $d \geq 1$, the set

$$
\left\{u \in U(\bar{k}) \mid[k(u): k] \leq d \text { and } R_{l}\left(\widehat{\psi_{u}}\right)=0\right\}
$$

is finite.

Proof. The proof of a) is analogous to that of Corollary 12.3; we apply Lemma 12.17 to the extension $\mathcal{E}(\psi)$ defined by $R_{l}\left(\widehat{\psi_{U}}\right)$ :

$$
0 \rightarrow H_{l}^{1}\left(\psi_{U}\right) \rightarrow \mathcal{E}(\psi) \rightarrow \pi^{*} H_{l, \mathrm{tr}}^{2}(S) \rightarrow 0
$$

By Proposition 11.1 a), the left term is isomorphic to the local system $\underline{E}$ of vanishing cycles, and the hypothesis of Lemma 12.17 is verified by part b) of this proposition. (Actually, $\underline{E}$ remains irreducible when restricted to any open subgroup of the monodromy.) Therefore $\mathcal{E}(\psi)$ is GLP. b) now follows from a), Theorem 12.16 and Theorem 11.5.

Theorem 12.21. Suppose $k$ finitely generated over $\mathbf{Q}$. Then, for any $d \geq 1$, the set

$$
\begin{aligned}
\operatorname{Exc}(\psi)=\{u \in U(\bar{k}) \mid[k(u): k] \leq d & \text { and } \widehat{\psi_{u}}=0 \text { in } \\
& \mathcal{M}_{\text {alg }}(k(u))\left(h_{1}\left(\psi_{u}\right), t_{2}\left(S_{k(u)}\right)\right\}
\end{aligned}
$$


is finite. So is the corresponding set for the condition

$$
\left(\psi_{u}\right)_{\#}=0 \text { in } \operatorname{Griff}\left(C_{u} \times_{k} S\right) \otimes \mathbf{Q} \text {. }
$$

Let $K$ be a field containing $\bar{k}$ (e.g., $K=\mathbf{C}$ ). With notation as in Theorem 12.5, the set $\operatorname{Exc}^{\mathcal{M}}\left(\psi_{K}\right)$ is contained in $U(\bar{k})$; in particular, it is countable.

Proof. This follows from Propositions 12.19, 12.20 and 9.3 and (for the last statement) from Lemma 12.18.

\subsection{Example: K3 surfaces.}

Corollary 12.22. In Theorem 12.21, assume that $S$ is a K3 surface and let $T_{u}=C_{u} \times S$. Then, for $u \notin \operatorname{Exc}(\psi)$, Murre's algebraic representative $\mathrm{Ab}^{2}\left(T_{u}\right)$ is isogenous to $J\left(C_{u}\right) \otimes \mathrm{NS}_{S}$.

Proof. Since $h_{1}(S)=h_{3}(S)=0$, we have $h_{1}\left(C_{u}\right)=h_{1}\left(\psi_{u}\right)$ and

$$
H_{l}^{3}\left(T_{u}\right)=H_{l}^{1}\left(\psi_{u}\right) \otimes H_{l}^{2}(S)
$$

hence

$$
N^{1} H_{l}^{3}\left(T_{u}\right)=H_{l}^{1}\left(\psi_{u}\right) \otimes \mathrm{NS}_{S}=H_{l}^{1}\left(C_{u}\right) \otimes \mathrm{NS}_{S}
$$

and the claim. 


\section{Appendix A. A Direct proof of Theorem 1}

Let $X \in \mathbf{S m}^{\text {proj }}(k)$. Recall from [79, Def. 1.5] the Albanese scheme $\mathcal{A}_{X}$ of $X$ : it is a $k$-group scheme locally of finite type, sitting in an extension

$$
0 \rightarrow \mathrm{Alb}_{X} \rightarrow \mathcal{A}_{X} \rightarrow \mathrm{Z} \pi_{0}(X) \rightarrow 0
$$

where $\mathrm{Alb}_{X}$ is the Albanese variety of $X$ and $\pi_{0}(X)$ is the scheme of constants of $X$. In [57, (8.1)], we aggregated the degree map and the Albanese map from the Chow group of 0-cycles of $X$ into a single homomorphism

$$
C H_{0}(X) \stackrel{a_{X}^{k}}{\longrightarrow} \mathcal{A}_{X}(k)
$$

We write

$$
T(X)=\operatorname{Ker} a_{X}^{k}
$$

(the Albanese kernel).

Let $Y$ be another smooth projective $k$-variety. As in $[57,(8.1 .3)]$ the homomorphism (A.2) and the universal property of $\mathcal{A}_{X}$ yields a map

$$
C H_{0}\left(X_{k(Y)}\right) \stackrel{a_{Y, X}}{\longrightarrow} \mathcal{A}_{X}(k(Y))=\operatorname{Hom}\left(\mathcal{A}_{Y}, \mathcal{A}_{X}\right) .
$$

By [57, Prop. 8.2.1], (A.3) makes $X \mapsto \mathcal{A}_{X}$ a functor from the category Chow $^{\circ}$ of birational Chow motives (with integral coefficients) to the category $\mathbf{A b S}$ of abelian schemes; a fortiori it is a functor on Chow $^{\text {eff }}$. In other words, the composite map

(A.4) $C H^{\operatorname{dim} X}(Y \times X) \rightarrow C H_{0}\left(X_{k(Y)}\right) \stackrel{a_{Y, X}}{\longrightarrow} \operatorname{Hom}\left(\mathcal{A}_{Y}, \mathcal{A}_{X}\right)=\mathcal{A}_{X}(Y)$

$[57,(8.1 .2)]$ is compatible with the composition of correspondences.

The morphism (A.2) is almost split. Namely, in [57, Prop. 8.2.1 and Th. 8.2.4] we prove that the functor $a_{\mathbf{Q}}: \mathbf{C h o w}^{\mathrm{o}}(k, \mathbf{Q}) \rightarrow \mathbf{A b S}(k, \mathbf{Q})$ is full, essentially surjective and has a fully faithful right adjoint $\rho$, whose essential image is the thick subcategory $\mathbf{C h o w}_{<1}^{\mathrm{o}}(k, \mathbf{Q})$ of Chow $^{\circ}(k, \mathbf{Q})$ generated by birational motives of curves. This yields:

Proposition A.1. Let $X \in \mathbf{S m}^{\text {proj }}(k)$. Then there exist an integer $n>0$ and, for any connected $Y \in \mathbf{S m}^{\text {proj }}(k)$, a homomorphism $\sigma_{Y}$ : $\mathcal{A}_{X}(k(Y)) \rightarrow C H_{0}\left(X_{k(Y)}\right)$ such that

(i) $\sigma_{Y}$ is natural in $Y$ for the action of correspondences (in $\mathbf{C h o w}^{\mathrm{o}}$ ).

(ii) $a_{X}^{k(Y)} \sigma_{Y}=n$ for any $Y$.

Proof. Let $M=\rho\left(\mathcal{A}_{X}\right)$. The unit map of the adjunction yields a morphism $\pi: h^{\mathrm{o}}(X) \rightarrow M$, where $h^{\mathrm{o}}(X) \in \operatorname{Chow}^{\mathrm{o}}(k, \mathbf{Q})$ is the birational motive of $X$, which induces an isomorphism $a_{\mathbf{Q}}(\pi): \mathcal{A}_{X} \stackrel{\sim}{\rightarrow} \mathcal{A}_{M}$ in $\operatorname{AbS}(k, \mathbf{Q})$. By the fullness of $a_{\mathbf{Q}}$, there exists a morphism $\sigma_{0}: M \rightarrow$ 
$h^{0}(X)$ such that $a_{\mathbf{Q}}\left(\sigma_{0}\right)$ is the inverse of $a_{\mathbf{Q}}(\pi)$. Write $M$ as a direct summand of the birational motive of a (not necessarily connected) curve $C$. Then $\sigma_{0}$ is induced by an algebraic correspondence from $C$ to $X$ with rational coefficients. There is an integer $n_{1}>0$ such that $\sigma_{1}=n_{1} \sigma_{0}$ has integral coefficients. Since $C H_{0}\left(C_{K}\right) \rightarrow \mathcal{A}_{C}(K)$ is injective with cokernel killed by some universal integer $n_{2}$ (see [57, Lemma 8.2.3]), $\sigma=n_{2} \sigma_{1}$ defines the desired system $\left(\sigma_{Y}\right)$.

Corollary A.2. a) There is an element $\xi \in T\left(X_{k(X)}\right)$ with the following property: for any $Y \in \mathbf{S m}^{\text {proj }}$ and any $y \in T\left(X_{k(Y)}\right)$, there is a morphism $y^{*}: T\left(X_{k(X)}\right) \rightarrow T\left(X_{k(Y)}\right)$ such that $y^{*} \xi=n y$. Here $n$ is as in Proposition A.1.

b) Suppose that $T\left(X_{\Omega}\right)=0$, where $\Omega$ is a universal domain. Then there is an integer $m>0$ such that $m T\left(X_{k(Y)}\right)=0$ for any $Y \in \operatorname{Sm}^{\text {proj }}(k)$.

Proof. Viewing $y$ as an element of $C H_{0}\left(X_{k(Y)}\right)=$ Chow $^{\circ}\left(h^{\circ}(Y), h^{\circ}(X)\right)$, it defines by pull-back a morphism $y^{*}: C H_{0}\left(X_{k(X)}\right) \rightarrow C H_{0}\left(X_{k(Y)}\right)$, which sends $T\left(X_{k(X)}\right)$ into $T\left(X_{k(Y)}\right)$ by the naturality of $a_{X}$. Let $\eta \in$ $C H_{0}\left(X_{k(X)}\right)$ be the class of the generic point: note that $y^{*} \eta=y$ (view $\eta$ as the identity endomorphism of $\left.h^{\circ}(X)\right)$. Define $\xi=n \eta-\sigma_{X} a_{X}^{k(X)}(\eta)$ where $\sigma_{X}$ is as in Proposition A.1. Then $\xi \in T\left(X_{k(X)}\right)$ and

$$
y^{*} \xi=n y-\sigma_{Y} a_{X}^{k(Y)} y=n y
$$

by the naturality of $a_{X}$ and $\sigma$.

b) The hypothesis implies that $\xi$ is torsion, hence the conclusion follows from a).

Remark A.3. As a converse to b), it follows from a) and Roǐtman's theorem that $T\left(X_{\Omega}\right)=0$ if $\xi$ is torsion. Unfortunately, $\xi$ is not canonically defined (it depends on the choice of the quasi-section $\sigma$ of Proposition A.1). It would be interesting to compute the exact exponent of $T\left(X_{k(Y)}\right)$ when $X$ is a surface with $q>0$ verifying Bloch's conjecture, as an extension of [55].

Composing (A.3) with restriction to the connected components, we get a map

$$
C H^{\operatorname{dim} X}(Y \times X) \rightarrow \operatorname{Hom}\left(\mathrm{Alb}_{Y}, \mathrm{Alb}_{X}\right)
$$

which is induced by the action of correspondences, hence factors through numerical equivalence. We have:

Lemma A.4. a) (cf. [57, (8.1.5)]) The restriction map $\operatorname{Hom}\left(\mathcal{A}_{Y}, \mathcal{A}_{X}\right)$ $\rightarrow \operatorname{Hom}\left(\mathrm{Alb}_{Y}, \mathrm{Alb}_{X}\right)$ sits in an exact sequence

$$
0 \rightarrow \operatorname{Hom}\left(\mathcal{A}_{\pi_{0}(Y)}, \mathcal{A}_{X}\right) \rightarrow \operatorname{Hom}\left(\mathcal{A}_{Y}, \mathcal{A}_{X}\right) \rightarrow \operatorname{Hom}\left(\mathrm{Alb}_{Y}, \mathrm{Alb}_{X}\right) .
$$


b) We have a complex

$$
0 \rightarrow \frac{T\left(X_{k(Y)}\right)}{T\left(X_{\pi_{0}(Y)}\right)} \stackrel{b}{\rightarrow} \frac{C H_{0}\left(X_{k(Y)}\right)}{C H_{0}\left(X_{\pi_{0}(Y)}\right)} \stackrel{a}{\rightarrow} \operatorname{Hom}\left(\mathrm{Alb}_{Y}, \mathrm{Alb}_{X}\right) \rightarrow 0
$$

which is split exact up to the integer $n$ of Proposition A.1.

The statement of b) means that there are maps $r, s$ such that $r b=n$ and $a s=n$.

Proof. For a), the exact sequence yields an exact sequence

$$
0 \rightarrow \operatorname{Hom}\left(\mathcal{A}_{\pi_{0}(X)}, \mathcal{A}_{Y}\right) \rightarrow \operatorname{Hom}\left(\mathcal{A}_{X}, \mathcal{A}_{Y}\right) \rightarrow \operatorname{Hom}\left(\operatorname{Alb}_{X}, \mathcal{A}_{Y}\right)
$$

and the latter group is isomorphic to $\operatorname{Hom}\left(\mathrm{Alb}_{X}, \mathrm{Alb}_{Y}\right)$. Then b) follows by a diagram chase.

Assume now that $Y$ is a curve $C$, that for simplicity we further suppose geometrically connected. The localisation exact sequences for the inclusions $U \times X \hookrightarrow C \times X$, where $U$ runs through the nonempty open subsets of $C$, yield in the limit an exact sequence

$$
\bigoplus_{c \in C_{(0)}} C H^{d-1}\left(X_{k(c)}\right) \rightarrow C H^{d}(C \times X) \rightarrow C H^{d}\left(X_{k(C)}\right) \rightarrow 0
$$

(where $d=\operatorname{dim} X$ ), which itself induces an exact sequence

$$
\bigoplus_{[E: k]<\infty} C H^{1}\left(C_{E}\right) \otimes C H^{d-1}\left(X_{E}\right) \rightarrow C H^{d}(C \times X) \rightarrow C H^{d}\left(X_{k(C)}\right) \rightarrow 0
$$

where the first map is given by external products and transfers. Together with Lemma A.4 b), this yields:

Proposition A.5. Suppose that $C$ is a geometrically connected curve, and let $d=\operatorname{dim} X$. Then we have a complex

$$
\begin{array}{r}
0 \rightarrow \frac{T\left(X_{k(C)}\right)}{T(X)} \rightarrow \frac{C H^{d}(C \times X)}{C H^{d}(X)+\sum_{[E: k]<\infty} \operatorname{Tr}_{E / k}\left(C H^{1}\left(C_{E}\right) \cdot C H^{d-1}\left(X_{E}\right)\right)} \\
\stackrel{a^{\prime}}{\rightarrow} \operatorname{Hom}\left(\mathrm{Alb}_{C}, \mathrm{Alb}_{X}\right) \rightarrow 0
\end{array}
$$

which is split exact up to the integer $n$ of Proposition A.1.

The proof of the next lemma is routine and omitted. 
Lemma A.6. Write $\pi: X \times C \rightarrow X$ for the projection. Assume that $C$ has a rational point $c$, and write $i: X \rightarrow C \times X$ for the corresponding inclusion. Consider the maps

$$
\begin{gathered}
\alpha: C H^{d}(X) \oplus C H^{1}(C) \otimes C H^{d-1}(X) \stackrel{\left(\pi^{*}, \cup\right)}{\longrightarrow} C H^{d}(C \times X) \\
\beta: C H^{d}(C \times X) \stackrel{\left(\begin{array}{c}
i^{*} \\
\pi_{*}
\end{array}\right)}{\longrightarrow} C H^{d}(X) \oplus C H^{d-1}(X) .
\end{gathered}
$$

Then

$$
\beta \circ \alpha=\operatorname{diag}\left(1_{C H^{d}(X)}, \operatorname{deg} \otimes 1_{C H^{d-1}(X)}\right) .
$$

The same holds a fortiori for coarser adequate equivalence relations.

Since (A.5) factors through numerical equivalence, so does $a^{\prime}$ in Proposition A.5. We have:

Proposition A.7. The map

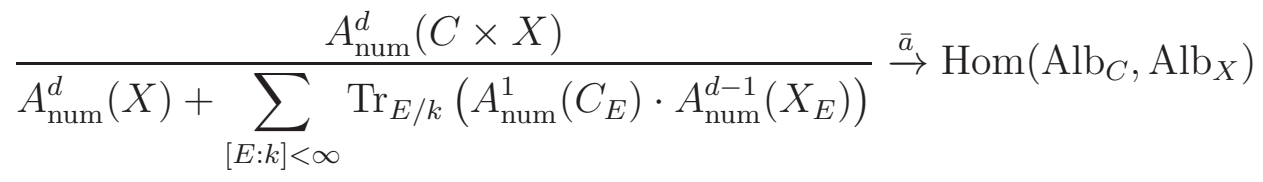

induced by the map a of Proposition A.5 has finite kernel and cokernel; its cokernel is killed by the integer $n$ of Proposition A.1 and its kernel is killed by

$$
I(C)=\operatorname{gcd}\left\{\operatorname{deg}(c) \mid c \in C_{(0)}\right\}
$$

(the index of $C$ ).

Proof. We first prove the analogous statement up to isogeny when replacing numerical equivalence with homological equivalence relative to some classical Weil cohomology, and assuming that $C$ has a rational point $c$. Then $A_{\text {hom }}^{1}\left(C_{E}\right)=\mathbf{Z}[c]$ for any $E$, so that

$$
\begin{aligned}
\frac{A_{\mathrm{hom}}^{d}(C \times X)}{A_{\mathrm{hom}}^{d}(X)+\sum_{[E: k]<\infty} \operatorname{Tr}_{E / k}\left(A_{\mathrm{hom}}^{1}\left(C_{E}\right) \cdot A_{\mathrm{hom}}^{d-1}\left(X_{E}\right)\right)} \\
=\frac{A_{\mathrm{hom}}^{d}(C \times X)}{A_{\mathrm{hom}}^{d}(X)+[c] \cdot A_{\mathrm{hom}}^{d-1}(X)} .
\end{aligned}
$$

Consider the commutative diagram

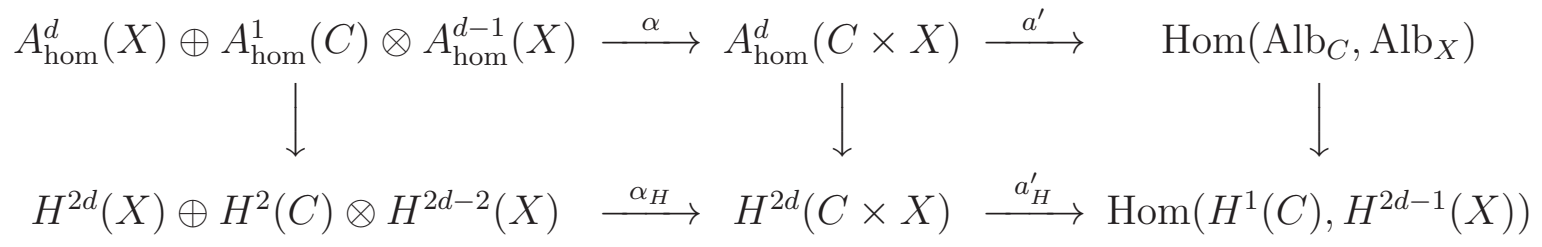


where $\alpha$ is as in Lemma A.6, the vertical maps are cycle class maps and $\alpha_{H}, a_{H}^{\prime}$ are constructed like $\alpha$ and $a^{\prime}$. The lower sequence is short exact, by the Künneth formula and Poincaré duality. Recall that $n \operatorname{Coker}\left(a^{\prime}\right)=0$ and that $\alpha$ has the retraction $\beta$. To obtain the exactness of the top sequence up to isogeny, it suffices to show that $\operatorname{Ker} a^{\prime} \cap \operatorname{Ker} \beta$ is torsion. But so is $\operatorname{Ker} \bar{a}_{H} \cap \operatorname{Ker} \beta_{H}$, where $\beta_{H}$ is defined analogously to $\beta$, thus the conclusion follows from the injectivity of the cycle class map.

We just proved that the map

$$
\frac{A_{\text {hom }}^{d}(C \times X)}{A_{\text {hom }}^{d}(X)+[c] \cdot A_{\text {hom }}^{d-1}(X)} \stackrel{\bar{a}}{\rightarrow} \operatorname{Hom}\left(\operatorname{Alb}_{C}, \operatorname{Alb}_{X}\right)
$$

has torsion kernel. A fortiori, so does the corresponding map for numerical equivalence. But $A_{\text {num }}^{d}(C \times X)$ is torsion-free, hence its quotient $\frac{A_{\text {num }}^{d}(C \times X)}{A_{\text {num }}^{d}(X)+[c] \cdot A_{\text {num }}^{d-1}(X)}$ by a direct summand is torsion-free too, and the map $\bar{a}$ of Proposition A.7 is injective in this case. The general case now follows by a transfer argument.

Remark A.8. As a byproduct of this proof, we see that the left hand side of the map in Proposition A.7 coincides up to torsion for homological and numerical equivalence.

Let $C H_{\text {num }}^{*}:=\operatorname{Ker}\left(C H^{*} \rightarrow A_{\text {num }}^{*}\right)$. Putting Propositions A.5 and A.7 together, we get:

Corollary A.9. If $C$ is a smooth connected curve having a 0-cycle of degree 1, the map of Proposition A.5 induces a homomorphism

$\frac{T\left(X_{k(C)}\right)}{T(X)} \rightarrow \frac{C H_{\mathrm{num}}^{d}(C \times X)}{C H_{\mathrm{num}}^{d}(X)+\sum_{[E: k]<\infty} \operatorname{Tr}_{E / k}\left(\operatorname{Pic}^{0}\left(C_{E}\right) \cdot C H^{d-1}\left(X_{E}\right) \oplus \operatorname{Pic}\left(C_{E}\right) \cdot C H_{\mathrm{num}}^{d-1}\left(X_{E}\right)\right)}$

with kernel and cokernel killed by $n$.

Note that all groups in the denominator of the right hand side, except perhaps $\operatorname{Pic}\left(C_{E}\right) \cdot C H_{\text {num }}^{d-1}\left(X_{E}\right)$, are made of cycles algebraically equivalent to 0 ; this is also true for the latter when $d=2$. Hence

Corollary A.10. For $d=2$, the map of Corollary A.9 induces a homomorphism with cokernel killed by $n$

$$
\frac{T\left(X_{k(C)}\right)}{T(X)} \rightarrow \operatorname{Griff}(C \times X) .
$$


In case $C$ does not have a 0 -cycle of degree 1 , one may have to multiply the map of Proposition A.5 by $I(C)$ to obtain a map like the one in Corollary A.10.

Corollary A.11. Let $d=2$, and let $\Omega / k$ be a universal domain. If $T\left(X_{\Omega}\right)=0$, then $n I(C) \operatorname{Griff}(C \times X)=0$ for any curve $C$, where $n$ is as in Proposition A.1.

Proof of Corollary 1. Let $T=C \times S$ : by Soulé [85, Th. 4.1] or [50, Th. 7.5.1], $C H^{2}(T) \otimes \mathbf{Q} \stackrel{\sim}{\rightarrow} A_{\text {num }}^{2}(T)$; by [50, Cor. 7.5.3], $C H^{2}(T)$ is finitely generated. It follows that $C H_{\text {num }}^{2}(T)$ is finite and that $T\left(S_{k(C)}\right)$ is finitely generated as a subquotient of $C H^{2}(T)$. Corollary A.9 then implies that $T\left(S_{k(C)}\right) / T(S)$ is finite. But $T(S)$ is finite, for example by Kato-Saito [58]. This concludes the proof.

\section{Appendix B. The Chow-Lefschetz isomorphism for $C \times S$}

Theorem B.1. Let $C, S$ be a curve and a surface over $k$, that we both assume geometrically connected, and let $T=C \times S$. Let $L^{\prime} \in$ $\operatorname{Pic}(C), L^{\prime \prime} \in \operatorname{Pic}(S)$ be classes of smooth hyperplane sections, and $L=L^{\prime} \otimes 1+1 \otimes L^{\prime \prime} \in \operatorname{Pic}(T)$ be the class of the hyperplane section attached to the corresponding Segre embedding. Then the morphism

$$
h_{4}(T) \stackrel{\cdot L}{\rightarrow} h_{2}(T)(1)
$$

is an isomorphism.

Proof. Write $h_{2}(S)=h_{2}^{\prime}(S) \oplus p_{2}(S)$, where $p_{2}(S)=\operatorname{Ker}\left(h_{2}(S) \stackrel{L^{\prime \prime}}{\rightarrow}\right.$ $h_{0}(S)(1)=\operatorname{Coker}\left(h_{4}(S)(-1) \stackrel{L^{\prime}}{\rightarrow} h_{2}(S)\right.$ (primitive part). Then we get decompositions of $h_{4}(T)$ and $h_{2}(T)$ into 4 summands:

$h_{4}(T)=h_{2}(C) \otimes p_{2}(S) \oplus h_{2}(C) \otimes h_{2}^{\prime}(S) \oplus h_{1}(C) \otimes h_{3}(S) \oplus h_{0}(C) \otimes h_{4}(S)$ $h_{2}(T)=h_{2}(C) \otimes h_{0}(S) \oplus h_{1}(C) \otimes h_{1}(S) \oplus h_{0}(C) \otimes p_{2}(S) \oplus h_{0}(C) \otimes h_{2}^{\prime}(S)$.

The matrices of $L^{\prime} \otimes 1$ and $1 \otimes L^{\prime \prime}$ with respect to these decompositions are respectively

$$
\left(\begin{array}{cccc}
0 & 0 & 0 & 0 \\
0 & 0 & 0 & 0 \\
i^{\prime} & 0 & 0 & 0 \\
0 & 0 & 0 & 0
\end{array}\right) \quad\left(\begin{array}{cccc}
0 & i_{1}^{\prime \prime} & 0 & 0 \\
0 & 0 & 0 & 0 \\
0 & 0 & i_{2}^{\prime \prime} & 0 \\
0 & 0 & 0 & i_{3}^{\prime \prime}
\end{array}\right)
$$

where $i^{\prime}: h_{2}(C) \otimes p_{2}(S) \rightarrow h_{0}(C)(1) \otimes p_{2}(S), i_{1}^{\prime \prime}: h_{2}(C) \otimes h_{2}^{\prime}(S) \rightarrow$ $h_{2}(C) \otimes h_{0}(S)(1), i_{2}^{\prime \prime}: h_{1}(C) \otimes h_{3}(S) \rightarrow h_{1}(C) \otimes h_{1}(S)(1)$ and $i_{3}^{\prime \prime}:$ $h_{0}(C) \otimes h_{4}(S) \rightarrow h_{0}(C) \otimes h_{2}^{\prime}(S)(1)$ are the isomorphisms induced by $L^{\prime}$ and $L^{\prime \prime}$. The sum of these two matrices is clearly invertible. 


\section{Appendix C. NORMALITy CRITERIA, AND MONODROMY OF VARIATIONS OF MIXED HODGE STRUCTURES}

by Yves André7

The aim of this appendix is to review and unravel some exactness criteria for a short sequence of linear algebraic groups, and to apply these criteria to the monodromy of variations of mixed Hodge structures.

\section{C.1. Normality criteria. Let}

$$
1 \rightarrow H \stackrel{q}{\rightarrow} G \stackrel{p}{\rightarrow} Q \rightarrow 1
$$

be a sequence of morphisms of linear algebraic groups (= geometrically reduced, i.e. smooth, affine group schemes of finite type) over a field $F$ of any characteristic.

C.1.1. In many concrete situations, one wishes to establish the exactness of (C.1) by tannakian duality, i.e. using properties of the categories of representations. There are well-known necessary and sufficient conditions for exactness on the left and on the right due to Saavedra (see e.g. [32, 2.21]), namely:

P1. $p$ is faithfully flat if and only $p^{*}\left(\operatorname{Rep}_{F} Q\right)$ is a tannakian subcategory of $\operatorname{Rep}_{F} G$ (equivalently: is a full subcategory closed under taking quotients (or subobjects)),

P2. $q$ is a closed embedding if and only if any object of $\operatorname{Rep}_{F} H$ is a subquotient of an object of $q^{*}\left(\operatorname{Rep}_{F} G\right)$.

Henceforth, we concentrate on exactness in the middle, assuming that $q$ is a closed embedding and $p$ is faithfully flat.

For any (rational, finite-dimensional) representation $V \in \operatorname{Rep}_{F} G$, we denote by $V^{H}$ the $F$-subspace of $H$-invariants. For any character $\chi: H \rightarrow \mathbb{G}_{m F}$, we denote by $V^{\chi}$ the $F$-subspace of $V$ where $H$ acts through $\chi$. We say that a character $\chi$ occurs in a representation of $G$ is there exists $V \in \operatorname{Rep}_{F} G$ such that $V^{\chi} \neq 0$.

An obvious exactness criterion is

Lemma C.1. (C.1) is exact if and only if the following conditions hold:

$\mathbf{H} 0$. In every $G$-representation $V, V^{H}=V^{\text {ker } p}$,

H1. $H$ is a normal subgroup of $G$.

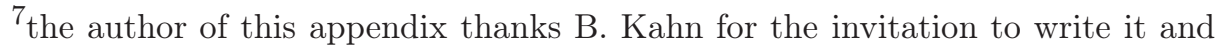
for his remarks and suggestions of improvement of the exposition.
} 
The "only if" part is trivial. For the "if" part, let $\bar{H}$ denote the smallest normal closed subgroup of $G$ containing $H$; then $\mathbf{H} 0$ implies $\bar{H}=\operatorname{Ker} p$ (consider a faithful representation of $G / \bar{H}$ ), and $\mathbf{H} 1$ is equivalent to $H=\bar{H}$.

Condition $\mathbf{H} 0$ alone is not enough to imply exactness ${ }^{8}$, as the example $Q=1, G=G L_{2}, H=\{$ upper triangular matrices $\}$ shows.

The lemma turns the exactness problem into expressing $\mathbf{H} 1$ in representation-theoretic terms. We shall discuss two such normality criteria.

C.1.2. The first criterion appears in [4, Lemma 1]:

Proposition C.2. $H$ is normal in $G$ if the following condition holds:

H2. For every character $\chi$ of $H$ occurring in a representation $V$ of $G, V^{\chi}$ is stable under $G$.

(The converse is true if $G$ is connected).

This is a consequence of Chevalley's theorem [70, 4.19]: $H$ is the stabilizer of a line $\ell$ in some representation $V$ of $G$. This line defines a character $\chi$. By assumption, $V^{\chi}$ is $G$-stable. Let $K$ be the kernel of $G \rightarrow G L\left(\operatorname{End}_{F} V^{\chi}\right)$. Then $H \subset K$. Conversely, $K$ commutes with any $F$-endomorphism of $V^{\chi}$, hence acts by scalars on $V^{\chi}$, hence stabilizes $\ell$. Therefore $H=K$.

(For the converse, the idea is that $G \times H \stackrel{(g, h) \mapsto g h g^{-1}}{\rightarrow} H \stackrel{\chi}{\rightarrow} \mathbb{G}_{m}$ defines a morphism from $G$ to the étale-locally constant group scheme $\operatorname{Hom}\left(H, \mathbb{G}_{m}\right)$ defined by a $\mathbf{Z}$-module of finite type; such a morphism factors through a finite quotient, hence is trivial if $G$ is connected.)

C.1.3. The second criterion appears in $[36 \text {, Th. A1 }]^{9}$ :

Proposition C.3. $H$ is normal in $G$ if and only if the following conditions hold:

H3. For every $V \in \operatorname{Rep}_{F} G, V^{H}$ is $G$-stable.

H4. $H$ is an observable subgroup of $G$, i.e. every representation of $H$ is contained in a representation of $G$.

One aim of this section is to highlight the relevance of the notion and of the theory of observability in the context of normality criteria ${ }^{10}$. Let us mention several useful characterizations of observability (due to

\footnotetext{
8 this is a common source of errors.

${ }^{9}$ precisely, the special case of loc. cit. for a sequence (C.1) in which $Q=G / \bar{H}$.

${ }^{10}$ actually, neither this term nor the theory of observability is mentioned in [36] (where the fact that a representation of a normal subgroup $H \triangleleft G$ is contained in a representation of $G$ is proved directly) - nor in any of the quoted references below about normality criteria.
} 
several authors, $c f$. [40] for a general reference where $F$ is algebraically closed, and [90, Th. 9] for the reduction to this case):

Proposition C.4. The following are equivalent:

(1) $H$ is observable in $G$,

(2) every character of $H$ occurs in a representation of $G$,

(3) $H$ is the isotropy group of some element in a representation of $G$,

(4) $G / H$ is quasi-affine.

Examples C.5. (1) A normal subgroup is observable (this follows from the characterization (4), cf. [70, 5.21]). In particular, $\bar{H}$ is observable in $G$, and $H$ is observable in $\bar{H}$ is and only if it is observable in $G$.

(2) If every character of $H$ occurring in a representation of $G$ is of finite order, then $H$ is observable in $G$ ( $H$ is the stabilizer of a line $\ell$ in a $G$-representation $V$; this defines a character, which is assumed to be of finite order $n$; then $H$ is the stabilizer of $\ell^{\otimes n}$ in $V^{\otimes n}$ (using the fact that the Segre diagonal map $\mathbf{P}(V) \rightarrow \mathbf{P}(V)^{n} \rightarrow \mathbf{P}\left(V^{\otimes n}\right)$ is an embedding, so that $\ell^{\otimes n}$ determines $\ell$ ), hence is the isotropy subgroup of any generator of $\ell^{\otimes n}$, and one concludes by (3)).

Let us come back to the proof Proposition C.3. The "only if" part follows from Example C.5 (1). Let us turn to the "if" part. Since $\bar{H}$ is observable (C.5 (2)), for any $W, W^{\prime} \in \operatorname{Rep}_{F} \bar{H}$, there is an injective morphism $W^{\prime} \hookrightarrow V^{\prime}$ and dually a surjective morphism $V \rightarrow W$ in $\operatorname{Rep}_{F} \bar{H}$, with $V, V^{\prime} \in \operatorname{Rep}_{F} G$. For any $H$-equivariant morphism $f$ : $W \rightarrow W^{\prime}$, the corresponding composition $g: V \rightarrow V^{\prime}$ is a morphism in $\operatorname{Rep}_{F} \bar{H}$ if and only if so is $f$ - and this holds true by $\mathbf{H} 3$ applied to $g \in \operatorname{Hom}\left(V, V^{\prime}\right)^{H}$. This shows that $\operatorname{Rep}_{F} \bar{H} \rightarrow \operatorname{Rep}_{F} H$ is full.

Let now $W$ be a quotient in $\operatorname{Rep}_{F} H$ of an object $V \in \operatorname{Rep}_{F} \bar{H}$. By $\mathbf{H} 4, W$ is contained in some $V^{\prime} \in \operatorname{Rep}_{F} \bar{H}$. Thus $W$ is the image of $V \rightarrow V^{\prime}$, which is a morphism in $\operatorname{Rep}_{F} \bar{H}$ by fullness. By $\mathbf{P} 1, H \hookrightarrow \bar{H}$ is faithfully flat, so that $H=\bar{H}$.

C.1.4. Combining C.3 and C.5 (2), one obtains the following new normality criterion:

Proposition C.6. $H$ is normal in $G$ if both $\mathbf{H} 3$ and the following condition hold:

H5. every character of $H$ occurring in a representation of $G$ is of finite order.

Remark C.7. There are other normality criteria in the literature, for instance [35, §4] (which is compared to [36, Th. A1] in [66]). See also $[13, \S 2]$ and $[69]$ in the context of non-neutral tannakian categories. 
Remark C.8. Propositions C.2, C.3, C.6 extend to arbitrary reduced affine group schemes over $F$, since for any object $V \in \operatorname{Rep}_{F} G$, the action of $G$ factors through a finite-dimensional quotient.

\section{C.2. Application to variations of mixed Hodge structures.}

C.2.1. Let $S$ be a smooth connected complex algebraic variety, with a base point $s$. We consider good graded-polarizable variations $V$ of mixed Q-Hodge structures over $S^{a n}$, whose underlying local system is defined over $\mathbf{Z}$ (i.e. there is a lattice in $V_{s}$ which is stable under $\pi_{1}(S, s)$ ), cf. [14]. They form a tannakian category $\mathcal{V}_{S}$, with fiber functor $\omega_{s}: \mathcal{V}_{S} \rightarrow$ $V e c_{\mathbf{Q}}$ given by the fiber at $s$. We denote by $T_{S, s}$ the corresponding tannakian group.

The forgetful $\otimes$-functor $\mathcal{V}_{S} \rightarrow \mathbf{L o c}_{S}$ given by the underlying local system of $\mathbf{Q}$-vector spaces gives rise to a morphism of tannakian groups $\Pi_{S, s} \rightarrow T_{S, s}$, and we denote by $M_{S, s}$ (for "monodromy") its image in $T_{S, s}$ (which is also the Zariski closure of the image of $\pi_{1}(S, s)$ in $T_{S, s}$ ).

On the other hand, constant variations (those for which the local system is constant) form a tannakian subcategory of $\mathcal{V}_{S}$, which can be identified via $\omega_{s}$ with the category MHS of graded-polarizable mixed Q-Hodge structures. We denote by MT (for "Mumford-Tate") its tannakian group, and by $p: T_{S, s} \rightarrow \mathrm{MT}$ the natural morphism. By $\mathbf{P} 1, p$ is faithfully flat.

C.2.2. The assumption that local systems are defined over $\mathbf{Z}$ has the following useful consequence:

Lemma C.9. Characters $\chi$ of $M_{S, s}$ occurring in a representation $V$ of $T_{S, s}$ are of finite order. More precisely, $\chi$ is either trivial or quadratic.

Indeed, $V$ has a lattice stable under $\pi_{1}(S, s)$, so that $V^{\chi}$ inherits a Z-structure on which $\pi_{1}(S, s)$ acts through $\chi$. Therefore $\chi$ is either trivial or quadratic since $\mathbf{Z}^{\times}=\{ \pm 1\}$.

C.2.3. "Good" (a.k.a. "admissible") is a condition on the behaviour at infinity of the variation ${ }^{11}$, which ensures that the so-called "theorem of the fixed part" holds:

\footnotetext{
${ }^{11}$ This condition is automatically satisfied in the case of pure Hodge structures, and is known to hold for variations of geometric origin. For a simple counterexample, inspired by [84], when this condition is not fulfilled, see [4, p.12]. For a exhaustive discussion, $c f$. [14].
} 
Theorem C.10. [84] $i$ ) For any $V \in \operatorname{Rep}_{\mathbf{Q}} T_{S, s}$ (corresponding to a variation $\mathbf{V} \in \mathcal{V}_{S}$ ), the fixed part $W:=V^{M_{S, s}}$ is stable under $T_{S, s}$ (i.e. is the fiber at $s$ of a subvariation $\mathbf{W}$ of $\mathbf{V}$ ).

ii) Moreover, it is a representation of $\mathrm{MT}$ (i.e. $\mathbf{W}$ is a constant variation).

C.2.4. The main result of this appendix is the following (which is a variation on $[4 \text {, Th. } 1]^{12}$ and is mentioned in $\left.[5, \S 1.4]\right)$ :

Theorem C.11. The sequence of affine group schemes over $\mathbf{Q}$

$$
1 \rightarrow M_{S, s} \stackrel{q}{\rightarrow} T_{S, s} \stackrel{p}{\rightarrow} \mathrm{MT} \rightarrow 1
$$

is split exact.

Proof. Part ii) of Theorem C.10 shows that Condition $\mathbf{H} 0$ of Lemma C.1 holds. To check H1, we use Proposition C.6 (and Remark C.8): part $i$ ) of Theorem C.10 shows that $\mathbf{H} 3$ holds, and Lemma C.9 shows that $\mathbf{H} 5$ holds. We conclude that (C.2) is exact. The $\otimes$-functor $\mathbf{V} \rightarrow$ $\mathrm{V}_{s}: \mathcal{V}_{S} \rightarrow$ MHS provides a canonical splitting of (C.2).

Remark C.12. Instead of Proposition C.6, one can alternatively use Proposition C.2 (which avoids the theory of observability) at the cost of a more delicate argument. Let $\chi$ be a character occurring in a representation $V$ of $T_{S, s}$ (corresponding to an object $\mathbf{V} \in \mathcal{V}_{S}$ ). If $\chi$ is trivial, $V^{\chi}$ is stable under $T_{S, s}$ by Theorem C.10. Otherwise, by Lemma C.9, there is a double étale covering $S^{\prime} \rightarrow S$ which kills $\chi$. Let $s^{\prime}$ be one of the two points of $S^{\prime}$ above $s$, and let us look at the pull-back $\mathbf{V}_{S^{\prime}} \in \mathcal{V}_{S^{\prime}}$ and at its fiber at $s^{\prime}$, identifying $V=\omega_{s}(\mathbf{V})$ and $\omega_{S^{\prime}}\left(\mathbf{V}_{S^{\prime}}\right)$.

One has $V^{M_{S^{\prime}, s^{\prime}}}=V^{M_{S, s}} \oplus V^{\chi}$ as $M_{S, s}$-representations. By Theorem C.10, this is the fiber at $s^{\prime}$ of a constant subvariation of $\mathbf{V}_{S^{\prime}}$, and since its underlying local system comes from a local system over $S^{a n}$, it is actually of the form $\mathbf{W}_{S^{\prime}}$ for a subvariation of $\mathbf{V}$ (this follows from the definition of a variation of mixed Hodge structures). Hence $V^{M_{S, s}} \oplus V^{\chi}$ is stable under $T_{S, s}$, and we may substitute it to the original $V$; in other words, we may assume that $M_{S, s}$ acts by $\mathbf{Z} / 2$ on $V$, and denoting by a subscript \pm the \pm 1 -eigenspaces, we have to show that $V_{-}$is stable under $T_{S, s}$.

\footnotetext{
${ }^{12}\left[4\right.$, Th. 1] deals with the Mumford-Tate group a general fiber $\mathbf{V}_{s}$ instead of the tannakian group of the variation $\mathbf{V}$, and with the connected monodromy group. Actually, it can be shown that the Mumford-Tate group of $\mathbf{V}_{s}$ coincides with the image of $T_{S, s}^{0}$ (using [4, Lemma 4], a variation on a result of Deligne in the mixed case), and that $\left[4\right.$, Th. 1] can be interpreted as the normality of $M_{S, s}^{0}$ in $T_{S, s}^{0}$.
} 
Since $\operatorname{Hom}_{\mathbf{Z} / 2}\left(V_{+}, V_{-}\right)=\operatorname{Hom}_{\mathbf{Z} / 2}\left(V_{-}, V_{+}\right)=0$, the $T_{S, s^{-}}$equivariant exact sequence

$$
0 \rightarrow V_{+} \rightarrow V \rightarrow V / V_{+} \rightarrow 0
$$

has a unique $\mathbf{Z} / 2$-equivariant splitting, and we have to show that this splitting is $T_{S, s}$-equivariant. For this, we apply (C.3) to the dual representation $V^{\vee}$, and compare this exact sequence to the $\left(T_{S, s}\right.$-equivariant) dual exact sequence of (C.3) for $V$ : their $\mathbf{Z} / 2$-equivariant splittings both correspond to the decomposition $V^{\vee}=\left(V^{\vee}\right)_{+} \oplus\left(V^{\vee}\right)_{-}$. Therefore the ( $T$-equivariant) morphisms

$$
\left(V^{\vee}\right)_{+} \rightarrow\left(V_{+}\right)^{\vee}, \quad\left(V / V_{+}\right)^{\vee} \rightarrow V^{\vee} /\left(V^{\vee}\right)_{+}
$$

are bijective and we get the claim for $V^{\vee}$, hence for $V^{13}$.

C.2.5. Let $S^{\prime}$ be a finite Galois étale covering of $S$, with group $\Gamma$, and $s^{\prime}$ a point of $S^{\prime}$ above $s$.

Then $\Gamma$ acts as a finite group of (anti)auto-equivalences of $\mathbf{L o c}_{S^{\prime}}$ and of $\mathcal{V}_{S^{\prime}}{ }^{14}$. The tannakian categories given by looking at objects and morphisms fixed by $\Gamma$ are equivalent to $\operatorname{Loc}_{S}$ and of $\mathcal{V}_{S}$ respectively. In particular, taking sums of conjugates by elements of $\Gamma$, one sees by P2 that $\Pi_{S^{\prime}, s^{\prime}} \rightarrow \Pi_{S, s}$ and $T_{S^{\prime}, s^{\prime}} \rightarrow T_{S, s}$ are closed embeddings. One has an exact sequence

$$
1 \rightarrow \Pi_{S^{\prime}, s^{\prime}} \rightarrow \Pi_{S, s} \rightarrow \Gamma \rightarrow 1 .
$$

On the other hand, any representation $V$ of $\Gamma$ admits a $\Gamma$-invariant lattice and, by averaging some scalar product, carries a $\Gamma$-invariant scalar product. This allows one to attach to $V$ an object ${ }^{15} \mathbf{V}$ of $\mathcal{V}_{S}$ with monodromy $\Gamma$ and whose fibers are trivial Hodge structures; whence a morphism $T_{S, s} \stackrel{p}{\rightarrow} \Gamma$ which, by $\mathbf{P} 1$, is faithfully flat.

Proposition C.13. The sequence of affine group schemes over $\mathbf{Q}$

$$
1 \rightarrow T_{S^{\prime}, s^{\prime}} \stackrel{q}{\rightarrow} T_{S, s} \stackrel{p}{\rightarrow} \Gamma \rightarrow 1
$$

is exact.

\footnotetext{
${ }^{13}$ this is the intrinsic version of the following shorter argument:

if $\underline{v}_{ \pm}$is a basis of $V_{ \pm}$with dual basis $\underline{v}_{ \pm}^{\vee},\left(\underline{v}_{+}^{\vee}, \underline{v}_{-}^{\vee}\right)$ is the dual basis of the basis $\left(\underline{v}_{+}, \underline{v}_{-}\right)$of $V$. By C.10, $T_{S, s}$ acts on $V$ by upper block-triangular matrices $T$, and on $V^{\vee}$ by upper block-triangular matrices ${ }^{t} T^{-1}$. Therefore the $T$ 's are block-diagonal.

${ }^{14}$ and even by (anti)automorphisms, if one replaces $\operatorname{Loc}_{S^{\prime}}$ and $\mathcal{V}_{S^{\prime}}$ by $\operatorname{Re} p_{\mathbf{Q}} \Pi_{S^{\prime}, s^{\prime}}$ and $\operatorname{Rep}_{\mathbf{Q}} T_{S^{\prime}, s^{\prime}}$ respectively.

${ }^{15}$ which could be called an Artin object in $\mathcal{V}_{S}$. When $S^{\prime}$ varies, Artin objects form a tannakian subcategory of $\mathcal{V}_{S}$ equivalent to $\operatorname{Rep}_{\mathbf{Q}} \pi_{1}^{e t}(S, s)$.
} 
Proof. Let $\mathbf{V}$ be an object of $\mathcal{V}_{S}$, with fiber $V$ at $s$. Then $V^{T_{S^{\prime}, s^{\prime}}}$ corresponds to the maximal constant subobject $\mathbf{W}^{\prime}$ of $\mathbf{V}_{S^{\prime}}$. By the exact sequence (C.4), the local system underlying $\mathbf{W}^{\prime}$ comes from a local system on $S^{a n}$, hence is of the form $\mathbf{W}_{S^{\prime}}$ for a subvariation $\mathbf{W}$ of $\mathbf{V}$ - which becomes constant and even trivial after pulling back to $S^{\prime}$. Such an object belongs to $p^{*} \operatorname{Rep}_{\mathbf{Q}} \Gamma$. This gives $\mathbf{H} 0$ and $\mathbf{H} 3$ for the sequence (C.5). $\mathbf{H} 5$ follows from Lemma C.9, and one concludes by Proposition C.6.

Combining the compatible exact sequences (C.4) and (C.5) with the connectedness of MT [4, Lemma 2], one gets:

Corollary C.14. $\pi_{0}\left(M_{S, s}\right)=\pi_{0}\left(T_{S, s}\right)=\pi_{1}^{e t}(S, s)$.

C.2.6. The first part of Lemma C.9 can be generalized as follows (Deligne [27, 4.2.9b]):

Corollary C.15. The radical of $M_{S, s}^{0}$ is prounipotent (equivalently: characters of $M_{S, s \mathbf{C}}$ are of finite order).

Proof. (sketch) We start with the extension of C.9 to real coefficients: Claim: characters $\chi$ of $\left(M_{S, s}\right)_{\mathbf{R}}$ occurring in a representation $V$ of $\left(T_{S, s}\right)_{\mathbf{R}}$ are of finite order (hence trivial or quadratic since $\mathbf{R}_{\text {tors }}^{\times}=$ $\{ \pm 1\})$.

Let $G$ (resp. $H$ ) be the image of $T_{S, s \mathbf{R}}$ in $G L(V)$. Replacing $S$ by a finite étale covering, we may assume that $H$ is connected, hence also $G$ (by Corollary C.14). By Theorem C.11, $H$ is normal in $G$, and by the converse of part of Proposition C.2, $V^{\chi}$ is $G$-stable, hence is the fiber at $s$ of a graded-polarizable variation of real mixed Hodge structures; passing to the maximal exterior power, the polarization forces $\chi$ to be of finite order. This proves the claim.

Let us now prove the corollary. The statement follows from the analogous statement about finite-dimensional quotients $M$ of $M_{S, s}^{0}$ : namely, that the reductive quotient $M^{\text {red }}$ (i.e. the quotient by its unipotent radical) is semisimple. Replacing $S$ by a finite etale covering, we may assume that $M^{\text {red }}$ is a quotient of $M_{S, s}$. It thus suffices to show that for any abelian semisimple representation $W$ of $M_{S, s}$, the image $A \subset G L(W)$ of $M_{S, s}$ is finite. Since $M_{S, s}$ is observable in $T_{S, s}, W$ extends to a representation $V$ of $T_{S, s}$. Changing notation, let $G$ (resp. $H$ ) be the image of $T_{S, s}$ (resp. $\left.M_{S, s}\right)$ in $G L(V)$. Then $A$ is an abelian reductive quotient of $H$. Due to the underlying $\mathbf{Z}$-structure, the image of $\pi_{1}(S, s)$ in $G L\left(W_{\mathbf{R}}\right)$ is discrete, and it suffices to shows that $A(\mathbf{R})$ is compact - equivalently: every character of $A_{\mathbf{R}}$ is of finite order -, which follows from the claim. 


\section{REFERENCES}

[1] J. Achter, S. Casalaina-Martin, C. Vial On descending cohomology geometrically, Compositio Math. 153 (2017) 1446-1478.

[2] A. Albano Infinite generation of the Griffiths group: a local proof, Thesis, Univ. Utah, 1986, unpublished.

[3] E. Ambrosi Specialization of Néron-Severi groups in positive characteristic, preprint, 2018, https://arxiv.org/abs/1810.06481.

[4] Y. André Mumford-Tate groups of mixed Hodge structures and the theorem of the fixed part, Compositio Math. 82 (1992), 1-24.

[5] Y. André, Groupes de Galois motiviques et périodes, Séminaire Bourbaki. Vol. 2015/2016. Exposés 1104-1119. Astérisque No. 390 (2017), Exp. No. 1104, 1-26.

[6] Y. André Pour une théorie inconditionnelle des motifs, Publ. Math. IHÉS 83 (1996), 5-49.

[7] Y. André On the Shafarevich and Tate conjectures for hyperkähler varieties, Math. Ann. 305 (1996), 205-248.

[8] Y. André, B. Kahn Construction inconditionnelle de groupes de Galois motiviques, C. R. Acad. Sci. Paris 234 (2002), 989-994.

[9] D. Arapura, A. Dhillon The motive of the moduli stack of G-bundles over the universal curve, Proc. Indian Acad. Sci. 118 (2008), 389-411.

[10] L. Barbieri-Viale et B. Kahn On the derived category of 1-motives, Astérisque 381 (2016), 1-254.

[11] F. Bardelli Curves of genus three on a general abelian threefold and the nonfinite generation of the Griffiths group, in Arithmetic of complex manifolds (Erlangen, 1988), Lecture Notes in Math. 1399, Springer, Berlin, 1989, 10-26.

[12] A. Beilinson, V. Vologodsky A DG guide to Voevodsky's motives, GAFA (Geom. Funct. Anal.) 17 (2008), 1709-1787.

[13] C. Bertolin Motivic Galois theory for 1-motives, Ann. Sci. Math. Québec 32 (2008), 105-124.

[14] P. Brosnan, F. El Zein Variations of mixed Hodge structure. Hodge theory, 333-409, Math. Notes 49, Princeton Univ. Press, Princeton, NJ, 2014.

[15] S. Bloch Torsion algebraic cycles and a theorem of Roitman, Compositio Math. 39 (1979), 107-127.

[16] S. Bloch Lectures on algebraic cycles (Second Ed.), New Math. Monographs 16, Cambridge Univ. Press, 2010.

[17] S. Bloch Algebraic cycles and values of L-functions, I, J. Reine Angew. Math. 350 (1984), 94-108.

[18] S. Bloch, V. Srinivas Remarks on correspondences and algebraic cycles, Amer. J. Math. 105 (1983), 1235-1253.

[19] A. Cadoret Motivated cycles under specialization, in "Groupes de Galois Géométriques et differentiel", P. Boalch and J.-M. Couveignes, éds., Sém. \& Congrès 27 (2012), SMF, 25-55.

[20] A. Cadoret Representations of étale fundamental groups and specialization of algebraic cycles, Proceedings volume in honor of Gerhard Frey's 75th birthday, Contemporary Math. To appear.

[21] A. Cadoret, Y. Tamagawa A uniform open image theorem for $\ell$-adic representations, I, Duke Math. J. 161 (2012), 2605-2634. 
[22] A. Cadoret, Y. Tamagawa $A$ uniform open image theorem for $\ell$-adic representations, II, Duke Math. J. 162 (2013), 2301-2344.

[23] G. Ceresa $C$ is not algebraically equivalent to $C^{-}$in its Jacobian, Ann. of Math. 117 (1983), 285-291.

[24] H. Clemens Homological equivalence, modulo algebraic equivalence, is not finitely generated, Publ. Math. IHÉS 58 (1983), 19-38 (1984).

[25] J.-L. Colliot-Thélène Descente galoisienne sur le second groupe de Chow: mise au point et applications, Doc. Math., Extra Volume: Alexander S. Merkurjev's Sixtieth Birthday (2015) 195-220.

[26] P. Deligne Cohomologie des intersections complètes, Exposé XI in Groupes de monodromie en géométrie algébrique (SGA 7), Vol. II (P. Deligne, N. Katz, eds.), Lect. Notes in Math., Springer, 39-61.

[27] P. Deligne Théorie de Hodge, II, Publ. Math. IHÉS 40 (1971), 5-57.

[28] P. Deligne Cohomologie étale: les points de départ, in Cohomologie étale (SGA 4 1/2) (P. Deligne et al), Lect. Notes in Math. 569, Springer, 1977, 4-75.

[29] P. Deligne La conjecture de Weil, I, Publ. Math. IHÉS 43 (1974), 273-307.

[30] P. Deligne La conjecture de Weil, II, Publ. Math. IHÉS 52 (1980), 137-252.

[31] P. Deligne Hodge cycles on abelian varieties (notes by J. Milne), Lect. Notes in Math. 900, Springer, 1982, 9-100.

[32] P. Deligne, J. Milne, Tannakian Categories, in Hodge Cycles, Motives, and Shimura Varieties, Lect. Notes in Math. 900, Springer, 1982, 101-228.

[33] A. Deninger, J. P. Murre Motivic decomposition of abelian schemes and the Fourier transform, J. reine angew. Math. 422 (1991), 201-219.

[34] H. Diaz The motive of the Fano surface of lines, C. R. Acad. Sci. Paris 354 (2016), 925-930.

[35] J. P. Dos Santos, The homotopy exact sequence for the fundamental group scheme and infinitesimal equivalence relations, Algebr. Geom. 2 (2015), no. $5,535-590$.

[36] H. Esnault, P. Hai, X. Sun, On Nori's fundamental group scheme. Geometry and dynamics of groups and spaces, 377-398, Progr. Math. 265, Birkhäuser, 2008.

[37] T. Ekedahl On the adic formalism, in The Grothendieck Festschrift II, Progr. Math. 87, Birkhäuser, 1990, 197-218.

[38] M. Fried, M. Jarden Field arithmetic (2nd ed.), Springer, 2005.

[39] S. Gorchinskiy, V. Guletskiǐ Motives and representability of algebraic cycles on threefolds over a field, J. Alg. Geom. 21 (2012), 347-373.

[40] F. Grosshans, Algebraic homogeneous spaces and invariant theory. Lecture Notes in Mathematics 1673. Springer, 1997.

[41] M. Green, P. A. Griffiths, K. Paranjape Cycles over fields of transcendence degree 1, Michigan Math. J. 52 (2004), 181-187.

[42] P. A. Griffiths On the periods of certain rational integrals, II, Ann. of Math. 90 (1969), 496-541.

[43] A. Grothendieck, J.-L. Verdier Conditions de finitude. Topos et sites fibrés. Applications aux questions de passage à la limite, Exp. VI in Théorie des topos et cohomologie étale des schémas (SGA 4), Vol. 2, Lect. Notes in Math. 270, Springer, 1972, 163-365.

[44] B. Harris Harmonic volumes, Acta Math. 150 (1983), 91-123. 
[45] B. Harris Homological versus algebraic equivalence in a Jacobian, Proc. Nat. Acad. Sci. U.S.A. 80 (1983), 1157-1158.

[46] U. Jannsen Motives, numerical equivalence and semi-simplicity, Invent. Math. 107 (1992), 447-452.

[47] U. Jannsen Motivic sheaves and filtrations on Chow groups, in Motives (U. Jannsen, S. Kleiman, J.-P. Serre, eds.), Proc. Symposia pure Math. 55 (1), 245-302.

[48] U. Jannsen Equivalence relations on algebraic cycles, in The arithmetic and geometry of algebraic cycles (Banff, AB, 1998), NATO Sci. Ser. C Math. Phys. Sci., 548, Kluwer, 2000, 225-260.

[49] J.-P. Jouanolou Cohomologie l-adique, Exposé VI in Cohomologie l-adique et fonctions L (SGA5), Lect. Notes in Math. 589, Springer, 1977, 251-281.

[50] B. Kahn Algebraic K-theory, algebraic cycles and arithmetic geometry, Handbook of $K$-theory. Vol. 1, 351-428, Springer, Berlin, 2005.

[51] B. Kahn The Brauer group and indecomposable (2,1)-cycles, Compositio Math. 152 (2016), 1041-1051.

[52] B. Kahn Classes de cycles motiviques étales, preliminary version to [53], https : / / arxiv.org/pdf/1102.0375v2.pdf.

[53] B. Kahn Classes de cycles motiviques étales, Algebra \& Number Theory 6 (2012), 1369-1407.

[54] B. Kahn Motifs et adjoints, Rendiconti Sem. Mat. Univ. Padova 139 (2018), 77-128.

[55] B. Kahn Torsion order of smooth projective surfaces (with an appendix of J.-L. Colliot-Thélène), Comment. Math. Helv. 92 (2017), 839-857.

[56] B. Kahn, J.P. Murre, C. Pedrini On the transcendental part of the motive of a surface, in Algebraic cycles and motives (for J.P. Murre's 75th birthday), LMS Series 344 (2), Cambridge University Press, 2007, 143-202.

[57] B. Kahn, R. Sujatha Birational motives, I: pure birational motives, Annals of $K$-theory 1 (2016), 379-440.

[58] K. Kato, S. Saito Unramified class field theory of arithmetical surfaces, Ann. of Math. 118 (1983), 241-275.

[59] T. Katsura, T. Shioda On Fermat varieties, Tôhoku Math. J. 31 (1979), 97-115.

[60] N. Katz Étude cohomologique des pinceaux de Lefschetz, Exposé XVIII in Groupes de monodromie en géométrie algébrique (SGA 7), Vol. II (P. Deligne, N. Katz, eds.), Lect. Notes in Math., Springer, 254-327.

[61] N. Katz Le théorème de Griffiths, Exposé XX in Groupes de monodromie en géométrie algébrique (SGA 7), Vol. II (P. Deligne, N. Katz, eds.), Lect. Notes in Math., Springer, 341-362.

[62] N. Katz Le niveau de la cohomologie des intersections complètes, Exposé XXI in Groupes de monodromie en géométrie algébrique (SGA 7), Vol. II (P. Deligne, N. Katz, eds.), Lect. Notes in Math., Springer, 363-400.

[63] N. Katz, W. Messing Some consequences of the Riemann hypothesis for varieties over finite fields, Invent. Math. 23 (1974), 73-77.

[64] S.I. Kimura Chow groups are finite-dimensional, in some sense, Math. Ann. 331 (2005), 173-201. 
[65] S. Kleiman Algebraic cycles and the Weil conjectures, in Dix exposés sur la cohomologie des schémas (A. Grothendieck, N.H. Kuiper éds.), North Holland-Masson, 1968, 359-386.

[66] C. Lazda, A. Pál, A homotopy exact sequence for overconvergent isocrystals, https://arxiv.org/abs/1704.07574.

[67] D. I. Lieberman Numerical and homological equivalence of algebraic cycles on Hodge manifolds, Amer. J. Math. 90 (1968), 366-374.

[68] K. Künnemann On the Chow motive of an abelian scheme, in Motives (U. Jannsen, S. Kleiman, J.-P. Serre, eds.), Proc. Symp. pure Math. 55 (1), 189-205.

[69] J. Milne Quotients of Tannakian categories, Theory Appl. Categ. 18 (2007), No. 21, 654-664.

[70] J. Milne Algebraic groups. The theory of group schemes of finite type over a field. Cambridge Studies in Advanced Mathematics 170. Cambridge University Press, Cambridge, 2017.

[71] D. Mumford Rational equivalence of 0-cycles on surfaces, J. Math. Kyoto Univ. 9 (1968), 195-204.

[72] J. P. Murre Applications of algebraic K-theory to the theory of algebraic cycles, in Algebraic geometry, Sitges (Barcelona), 1983, 216-261, Lecture Notes in Math. 1124, Springer 1985.

[73] J. P. Murre On the motive of an algebraic surface, J. Reine angew. Math. 409 (1990), 190-204.

[74] G. A. Mustafin Families of algebraic varieties and invariant cycles, English translation from Russian: Math. USSR Izv 27 (1986), 251-278.

[75] M. Nori Cycles on the generic abelian threefold, Proc. Indian Acad. Sci. Math. Sci. 99 (1989), 191-196.

[76] P. O'Sullivan Algebraic cycles on an abelian variety, J. reine angew. Math. $654(2011,1-81$.

[77] N. Otsubo On the Abel-Jacobi maps of Fermat Jacobians, Math. Z. 270 (2012), 423-444.

[78] K. Paranjape Curves on threefolds with trivial canonical bundle, Proc. Indian Acad. Sci. Math. Sci. 101 (1991), 199-213.

[79] N. Ramachandran Duality of Albanese and Picard 1-motives, K-Theory 22 (2001), 271-301.

[80] P. Samuel Relations d'équivalence en géométrie algébrique, Proc. ICM, 1958.

[81] C. Schoen Complex multiplication cycles on elliptic modular threefolds, Duke Math. J. 53 (1986), 771-794.

[82] A. Scholl Classical motives, in Motives (U. Jannsen, S. Kleiman, J.-P. Serre, eds.), Proc. Symp. Pure Math. 55 (I), 163-187.

[83] J.-P. Serre Lectures on the Mordell-Weil theorem, Springer, 1989-19901997.

[84] J. Steenbrink, S. Zucker Variation of mixed Hodge structure, I, Invent. Math. 80 (1985), 489-542.

[85] C. Soulé Groupes de Chow et $K$-théorie de variétés sur un corps fini, Math. Ann. 268 (1984), 317-345.

[86] Y. Tadokoro A nontrivial algebraic cycle in the Jacobian variety of the Klein quartic, Math. Z. 260 (2008), 265-275. 
[87] Y. Tadokoro A nontrivial algebraic cycle in the Jacobian variety of the Fermat sextic, Tsukuba J. Math. 33 (2009), 29-38.

[88] Y. Tadokoro Nontrivial algebraic cycles in the Jacobian varieties of some quotients of Fermat curves, Internat. J. Math. 27 (2016), no. 3, 1650027, $10 \mathrm{pp}$.

[89] T. Terasoma Complete intersections with middle Picard number 1 defined over Q, Math. Z. 189 (1985), 289-296.

[90] N. Q. Thang, D. P. Bac, Some rationality properties of observable groups and related questions Illinois J. Math. 49 (2005), no. 2, 431-444.

[91] J. Top Hecke L-series related with algebraic cycles or with Siegel modular forms, Doctoral thesis, Univ. Utrecht, 1989, unpublished.

[92] C. Vial Niveau and coniveau filtrations on cohomology groups and Chow groups, Proc. LMS 106 (2013), 410-444.

[93] C. Vial Projectors Projectors on the intermediate algebraic Jacobians, New York J. Math. 19 (2013) 793-822.

[94] V. Voevodsky Triangulated categories of motives over a field, in Cycles, transfers, and motivic homology theories, Ann. of Math. Stud. 143, Princeton Univ. Press, 2000, 188-238.

[95] C. Voisin Une approche infinitésimale du théorème de H. Clemens sur les cycles d'une quintique générale de $\mathbf{P}^{4}$, J. Algebraic Geom. 1 (1992), 157174.

[96] C. Voisin The Griffiths group of a general Calabi-Yau threefold is not finitely generated, Duke Math. J. 102 (2000), 151-186.

[97] C. Voisin Unirational threefolds with no universal codimension 2 cycle, Invent. Math. 201 (2015), 207-237.

[98] D. Zelinsky Some abelian threefolds with nontrivial Griffiths group, Compositio Math. 78 (1991), 315-355.

IMJ-PRG, Case 247, 4 Place Jussieu, 75252 Paris Cedex 05, France

E-mail address: bruno.kahn@imj-prg.fr

IMJ-PrG, Case 247, 4 Place Jussieu, 75252 Paris Cedex 05, France

E-mail address: yves.andre@imj-prg.fr 